\title{
EXISTENCE AND CONTINUITY OF EXPONENTIAL ATTRACTORS OF THE THREE DIMENSIONAL NAVIER-STOKES- $\alpha$ EQUATIONS FOR UNIFORMLY ROTATING GEOPHYSICAL FLUIDS*
}

\author{
BONG-SIK KIM ${ }^{\dagger}$ AND BASIL NICOLAENKO ${ }^{\ddagger}$
}

\begin{abstract}
Three dimensional (3D) Navier-Stokes- $\alpha$ equations are considered for uniformly rotating geophysical fluid flows (large Coriolis parameter $f=2 \Omega$ ). The Navier-Stokes- $\alpha$ equations are a nonlinear dispersive regularization of usual Navier-Stokes equations obtained by Lagrangian averaging. The focus is on the existence and global regularity of solutions of the 3D rotating NavierStokes- $\alpha$ equations and the uniform convergence of these solutions to those of the original 3D rotating Navier-Stokes equations for large Coriolis parameters $f$ as $\alpha \rightarrow 0$. Methods are based on fast singular oscillating limits and results are obtained for periodic boundary conditions for all domain aspect ratios, including the case of three wave resonances which yields nonlinear resonant limit $\alpha$-equations for $f \rightarrow \infty$. The existence and global regularity of solutions of resonant limit $\alpha$-equations is established, uniformly in $\alpha$. Bootstrapping from global regularity of the resonant limit $\alpha$-equations, the existence of a regular solution of the full 3D rotating Navier-Stokes- $\alpha$ equations for large $f$ for an infinite time is established. Then we prove the existence of exponential attractors of the 3D rotating Navier-Stokes- $\alpha$ equations $(\alpha \neq 0)$ and the convergence of the exponential attractors to those of the original 3D rotating Navier-Stokes equations $(\alpha=0)$ for $f$ large but fixed as $\alpha \rightarrow 0$. All the estimates are uniform in $\alpha$, in contrast with previous estimates in the literature which blow up as $\alpha \rightarrow 0$.
\end{abstract}

Key words. Navier-Stokes- $\alpha$ equations, fast singular oscillating limits, operator splitting, uniform regularity estimates in $\alpha$, continuity of exponential attractors

AMS subject classifications. 35Q30, 37L30, 76D03, 76U05

\section{Introduction}

Geophysical fluid dynamics study flows for which the dynamics of the earth's rotation (Coriolis force) and stratification (buoyancy force) play important roles. These properties distinguish them from general fluids and make them special in the field of fluid dynamics. The dynamics of geophysical fluids admit motions which can vary on different time and length scales from a few kilometers to thousands of meters and from a few days to hundreds of years, and the ratio of vertical and horizontal length scales changes from one phenomena to another. Computation of this class of motions using a model based on the fundamental three-dimensional (3D) Navier-Stokes equations is prohibitive due to severe accuracy and time step restrictions. To resolve these restrictons, a number of modifications have been proposed. The Navier-Stokes- $\alpha$ model is one of the promising approximate models.

Marsden, Holm, and Ratiu introduced the $\alpha$-model for the mean motion of the incompressible ideal fluid flows (without viscosity) in three dimensions, including rotation and stratification ([25] and [26]). Later, S. Chen et al added viscosity to them ([15] and [16]). This is a dispersive regularization of Euler/Navier-Stokes equations, based on the Euler-Poincaré equations (see [27] for details) and Lagrangian averaging. In this model, the amplitude of the rapid fluctuations introduces a length scale, $\alpha>0$, below which wave activity is filtered by both linear and nonlinear dispersion.

\footnotetext{
${ }^{*}$ Received: October 24, 2005; accepted (in revised version): April 14, 2006. Communicated by Francois Golse.

${ }^{\dagger}$ Department of Mathematics, The Ohio State University, Columbus, OH 434210, USA (kim@math.ohio-state.edu).

$\ddagger$ Department of Mathematics and Statistics, Arizona State University, Tempe, AZ 85287, USA (byn@stokes.la.asu.edu).
} 
Inspired by successful computational comparison with empirical data ( [14], [15], [16], [17] ) of the $\alpha$-model for turbulent channel and pipe flows, further studies of the model in the context of turbulence modeling have been done and pointed to the $\alpha$ model to be a good subgrid large eddy simulation model of turbulence ([20], [21], [24], $[31])$. In spite of these encouraging results there remain important issues on the range of validity of the $\alpha$ model:

- Under what conditions does the $\alpha$-model approximate the 3D Navier-Stokes equations of geophysics in turbulent regimes?

- How do the dynamical attractors of solutions to the $\alpha$-model converge to those of the original 3D Navier-Stokes equations as $\alpha \rightarrow 0$ ?

- What are the scaling laws between $\alpha$ and important dimensionless numbers characterizing turbulent geophysical flows (e.g., Rossby, Froude, Reynolds number, etc.) which optimize the convergence?

This paper explores some of these issues in the context of the 3D Rotating NavierStokes (RNS) equations of geophysics. The corresponding 3D Rotating Navier-Stokes$\alpha$ (RNS- $\alpha)$ equations without buoyancy effects are

$$
\begin{aligned}
\frac{\partial V}{\partial t}+(U \cdot \nabla) V+V_{j} \nabla U^{j}+f e_{3} \times U & =-\nabla \pi+\nu \Delta V+F \\
\nabla \cdot V & =0 \\
\left.V(t, x)\right|_{t=0} & =V(0, x)=V(0)
\end{aligned}
$$

where

$$
\begin{aligned}
V(t, x) & =\left(V_{1}, V_{2}, V_{3}\right) \quad \text { the actual velocity, } \\
U(t, x) & =\left(1-\alpha^{2} \Delta\right)^{-1} V(t, x) \quad \text { the filtered or regularized velocity, } \\
\pi & =\frac{p}{\rho}-\frac{1}{2}|U|^{2}-\frac{\alpha^{2}}{2}|\nabla U|^{2} \text { a modified pressure. }
\end{aligned}
$$

Here $x=\left(x_{1}, x_{2}, x_{3}\right), f=2 \Omega$ is the Coriolis parameter, $F=\left(F_{1}, F_{2}, F_{3}\right)$ is a divergence free force, $\nu>0$ is the kinetic viscosity, $\rho$ is the fluid density, and $p$ is the pressure. For simplicity we will assume the forcing term to be time independent; that is, $F \equiv F(x)$.

The system is considered subject to periodic boundary conditions in a lattice $Q=\left[0,2 \pi a_{1}\right] \times\left[0,2 \pi a_{2}\right] \times\left[0,2 \pi a_{3}\right]$, as well as stress-free boundary conditions in the vertical. The corresponding function spaces are Fourier-Sobolev spaces of periodic functions, $H^{s}, s \geq 0$, with the norm

$$
\|u\|_{s}^{2}=\sum_{n \in Z^{3}}|\check{n}|^{2 s}\left|u_{n}\right|^{2}
$$

where $n=\left(n_{1}, n_{2}, n_{3}\right) \in Z^{3}$ is a wave number and $\check{n}=\left(\check{n}_{1}, \check{n}_{2}, \check{n}_{3}\right)$ with $\check{n}_{j}=n_{j} / a_{j}$ for $j=1,2,3$. We set $a_{1}=1$ without loss of generality. Problems addressed are

(i) whether the 3D RNS- $\alpha$ equations possess existence and global regularity of solutions with uniform regularity estimates in $\alpha$, and

(ii) what are the constraints on $\alpha$ for $\alpha$-trajectories to effectively shadow real trajectories of the 3D RNS equations, especially, with respect to important geophysical functionals such as enstrophy.

There have been several efforts in this direction. E. Titi, C. Foias, D. Holm, ([22]) proved the existence and global regularity of solutions of the 3D Navier-Stokes- $\alpha$ 
equations (without rotation). Their regularity estimates, however, are for filtered (or regularized) velocity, $U$, but not for the actual velocity, $V$. In particular, such estimates strongly depend on $\alpha$ and blow up as $\alpha \rightarrow 0$. This is an obstacle to establish the uniform and strong convergence of the solution of 3D NS- $\alpha$ equations when $\alpha$ vanishes. Instead, they proved the weak convergence of such solutions to some weak Leray solutions of Navier-Stokes equations as $\alpha \rightarrow 0$. The uniform controllability of $\alpha$ is at the heart of the difficulty. This stems from the fact that the Navier-Stokes- $\alpha$ equations (when $f=0$ ) lack a uniform spatial $L^{2}$-norm estimate (a Lyapunov functional for the set of Leray solutions, [30]) of the unfiltered velocity $V$. The corresponding Lyapunov functional for the three dimensional Navier-Stokes- $\alpha$ equations is

$$
|<V, U>|^{2}=\int V \cdot U d x=\int|U|^{2} d x+\alpha^{2} \int|\nabla U|^{2} d x<K
$$

for any $U \in H^{1}$ relative to $V$ by the relation $U=\left(1-\alpha^{2} \triangle\right)^{-1} V$, where $K$ is a constant uniform in $\alpha$. For our purpose, we intend to establish the existence independent of $\alpha$ for $f$ large enough, which is necessary in constructing the uniform convergence of the solution of RNS- $\alpha$ equations. We follow the methods that A. Babin, A. Mahalov, B. Nicolaenko developed in their papers, [1]-[13].

For $\alpha=0$, this system bears on the classical geophysical equations, the 3D RNS equations. The existence and global regularity of the $3 \mathrm{D}$ RNS equations for large $f$ have been established by Babin, Mahalov and Nicolaenko (BMN; [1], [2], [5], [6], [7]). They applied to Navier-Stokes and Euler equations with fast oscillations the nonlinear averaging methods of geometric optics and hyperbolic systems ([1]-[13]). With such an approach, there are three foremost issues with the analysis of the 3D RNS- $\alpha$ equations of geophysics for a large parameter $f$.

(i) First, the nature of the resonant limit $\alpha$-equations as $f \rightarrow \infty$ and the regularity of their solutions. This is a non-trivial process in which the 3 -wave resonant structure must be rigorously analyzed.

(ii) Second, the convergence of solutions of the 3D RNS- $\alpha$ equations to those of the resonant limit $\alpha$-equations.

(iii) Finally, bootstrapping from analysis of the first two questions the infinite time regularity of solutions of the equations for large $f$ but finite, $\alpha$ bounded.

Following this program, we, however, encounter substantial technical difficulties due to the fact that the Lagrangian averaged $\alpha$-model lacks a uniform spatial $L^{2}$-norm of the unfiltered velocity $V$. The best uniform in $\alpha$ estimates in this work turn out to be restricted to $H^{\beta}, \beta>5 / 2$. Based on the existence and regularity results we establish the existence of exponential attractors of the 3D RNS- $\alpha$ equations. Then we prove the convergence of the exponential attractors of the 3D RNS- $\alpha$ equations to those of the original 3D rotating NSE, which also delineates the constraints of nonlinear scaling laws of parameter $\alpha$ in terms of enstrophy and others.

This paper is organized as follows:

- In section 2, we set up functional spaces and investigate basic properties of the 3D RNS- $\alpha$ equations.

- In section 3, we use techniques developed by Babin, Mahalov, and Nicolaenko ([1]-[14]) for fast singular oscillating limits of (1.1)-(1.3) as $f \rightarrow \infty$. We analyze resonant structure of the equations, which leads to operator splitting. Through the splitting we obtain baroclinic and barotropic limit $\alpha$-equations.

- In section 4 , we establish the existence and global regularity of solutions of limit resonant $\alpha$-equations with uniform regularity estimates in $\alpha$. 
- In section 5, the local existence and regularity of solutions of the full 3D RNS- $\alpha$ equations are established in $H^{\beta}, \beta>5 / 2$. All estimates are uniform in $\alpha$ and independent from $f$.

- In section 6 , we establish the existence and global regularity of the 3D RNS- $\alpha$ equations with large $f$ in $H^{\beta}, \beta>5 / 2$. Regularity estimates are uniform in $\alpha$. The existence of absorbing sets in $H^{\beta}$ follows.

- In section 7 , we construct uniform exponential attractors of the $3 \mathrm{D}$ RNS- $\alpha$ equations in $H^{\beta}$.

- In section 8 , alternate form of the governing equations is derived, with the help of the operator $\left(\mathcal{R}_{\alpha}-I\right)$, where $\mathcal{R}_{\alpha}=\left(1-\alpha^{2} \Delta\right)^{-1}$ and $I$ is the identity. This operator plays a crucial role in proving the convergence of solutions. In appropriate spaces, the norm of the operator can be taken as small as needed, and it induces the uniform convergence as $\alpha \rightarrow 0$ in Fourier-Sobolev spaces. Then, we prove the uniform convergence theorem of exponential attractors in $L^{2}$ as $\alpha \rightarrow 0$.

- Finally, in section 9 , we conclude with remarks on the delination of constraints of $\alpha$ scaling, for uniform convergence, which are nonlinear with respect to $1 / f$ and the enstrophy $\|V\|_{1}$.

\section{Preliminaries}

We consider domain parameters, $a_{1}, a_{2}$ and $a_{3}$ bounded away from both zero and infinity. $V$ and $U$ are vector fields defined on $D=Q \times[0, T]$ for any $T>0$. Periodicity of the boundary conditions leads naturally to a Fourier representation of the fields, that is

$$
V=\sum_{n} V_{n} e^{i\left(n_{1} x_{1} / a_{1}+n_{2} x_{2} / a_{2}+n_{3} / a_{3}\right)}=\sum_{n} V_{n} e^{i \check{n} \cdot x},
$$

where $V_{n}$ 's are Fourier Coefficients. We introduce Fourier-Sobolev spaces of divergence free periodic vector fields

$$
H^{s}=\left\{u \in\left[L^{2}(Q)\right]^{3} \mid u=\sum_{n \in \mathbb{Z}^{3}} u_{n} e^{i \check{n} \cdot x}, u_{n}^{*}=u_{-n}, u_{0}=0, \check{n} \cdot u_{n}=0,\|u\|_{s}^{2}<\infty\right\},
$$

with the norm $\|\left. u\right|_{s} ^{2}=\sum_{n \in \mathbb{Z}^{3}}|\check{n}|^{2 s}\left|u_{n}\right|^{2}$. The corresponding inner product is denoted by $\langle\cdot, \cdot\rangle_{s}$. When $s=0, H^{0}=H$ and we denote $\langle\cdot, \cdot\rangle_{0}=\langle\cdot, \cdot\rangle$ and $\|\cdot\|_{0}=|\cdot|$ as the inner product and the corresponding norm in $H$, respectively. For $s=1$, we denote $\|\cdot\|_{1}=\|\cdot\|$ as the $H^{1}$ norm. We assume that

$$
\int_{Q} U(x, 0) d x=0 \quad \text { and } \quad \int_{Q} F(x) d x=0 .
$$

This yields $\int_{Q} V(x, t) d x=0$ for all $t \geq 0$, and allows for the use of Poincaré inequality.

We denote $P_{L}$ as the usual Leray projector and introduce an operator $\mathcal{R}_{\alpha}=(1-$ $\left.\alpha^{2} \Delta\right)^{-1}$, which is defined by $\mathcal{R}_{\alpha} v=\left(1-\alpha^{2} \Delta\right)^{-1} v$. We also define a bilinear operator $B_{\alpha}$ on divergence free vector fields by

$$
B_{\alpha}(u, v)=P_{L}\left[\left(\mathcal{R}_{\alpha} u \cdot \nabla\right) v+v_{j} \nabla\left(\mathcal{R}_{\alpha} u\right)_{j}\right] .
$$

Then $\mathrm{Eq}(1.1)$ can be rewritten in the form

$$
\frac{\partial V}{\partial t}+f P_{L} J P_{L} \mathcal{R}_{\alpha} V+\nu A V+B_{\alpha}(V, V)=F
$$


where $A=-P_{L} \Delta$ is the Stokes operator and $J$ is a rotation matrix given by

$$
J=\left(\begin{array}{ccc}
0 & -1 & 0 \\
1 & 0 & 0 \\
0 & 0 & 0
\end{array}\right) .
$$

Lemma 2.1. $B_{\alpha}(V, V)=P_{L}\left[\left(\mathcal{R}_{\alpha} V \cdot \nabla\right) V+V_{j} \nabla\left(\mathcal{R}_{\alpha} V\right)_{j}\right]=-P_{L}\left[\mathcal{R}_{\alpha} V \times \operatorname{curl} V\right]$.

Proof. Let $U=\mathcal{R}_{\alpha} V$. Using the identity $(a \cdot \nabla) b=\nabla(a \cdot b)-(b \cdot \nabla) a-a \times \operatorname{curl} b-$ $b \times \operatorname{curl} a$ we have

$$
(U \cdot \nabla) V+V_{j} \nabla U_{j}=\nabla(U \cdot V)-(V \cdot \nabla) U-U \times \operatorname{curl} V-V \times \operatorname{curl} U+V_{j} \nabla U_{j} .
$$

Notice that

$$
\begin{aligned}
& \left.(V \cdot \nabla) U-V_{j} \nabla U^{j}=\left[\begin{array}{c}
V_{j} \\
\frac{\partial U^{1}}{\partial x_{j}}-\frac{\partial U^{j}}{\partial x_{1}} \\
V_{j} \\
\frac{\partial U^{2}}{\partial x_{j}}-\frac{\partial U^{j}}{\partial x_{2}} \\
V_{j} \\
\frac{\partial U^{3}}{\partial x_{j}}-\frac{\partial U^{j}}{\partial x_{3}}
\end{array}\right]\right] \\
& =\left[\begin{array}{l}
V_{2}\left[\frac{\partial U^{1}}{\partial x_{2}}-\frac{\partial U^{2}}{\partial x_{1}}\right]+V_{3}\left[\frac{\partial U^{1}}{\partial x_{3}}-\frac{\partial U^{3}}{\partial x_{1}}\right] \\
V_{1}\left[\frac{\partial U^{2}}{\partial x_{1}}-\frac{\partial U^{1}}{\partial x_{2}}\right]+V_{3}\left[\frac{\partial U^{2}}{\partial x_{3}}-\frac{\partial U^{3}}{\partial x_{2}}\right. \\
V_{1}\left[\frac{\partial U^{3}}{\partial x_{1}}-\frac{\partial U^{1}}{\partial x_{3}}\right]+V_{2}\left[\frac{\partial U^{3}}{\partial x_{2}}-\frac{\partial U^{2}}{\partial x_{3}}\right]
\end{array}\right] \\
& =\left[\begin{array}{l}
-\left\{\begin{array}{l}
V_{2}\left[\frac{\partial U^{2}}{\partial x_{1}}-\frac{\partial U^{1}}{\partial x_{2}}\right]-V_{3}\left[\frac{\partial U^{1}}{\partial x_{3}}-\frac{\partial U^{3}}{\partial x_{1}}\right] \\
-\left\{\begin{array}{l}
V_{3}\left[\frac{\partial U^{3}}{\partial x_{2}}-\frac{\partial U^{2}}{\partial x_{3}}\right]-V_{1}\left[\frac{\partial U^{2}}{\partial x_{1}}-\frac{\partial U^{1}}{\partial x_{2}}\right] \\
-\left\{V_{1}\left[\frac{\partial U^{1}}{\partial x_{3}}-\frac{\partial U^{3}}{\partial x_{1}}\right]-V_{2}\left[\frac{\partial U^{3}}{\partial x_{2}}-\frac{\partial U^{2}}{\partial x_{3}}\right]\right.
\end{array}\right\}
\end{array}\right\}
\end{array}\right\} \\
& =-(V \times \operatorname{curl} U) \text {. }
\end{aligned}
$$

Thus

$$
V \times \operatorname{curl} U+(V \cdot \nabla) U-V_{j} \nabla U^{j}=[V \times \operatorname{curl} U]-[V \times \operatorname{curl} U]=0 .
$$

Since $P_{L}[\nabla(U \cdot V)]=0$, the result follows with the substitution of (2.4) into (2.3).

For the strong convergence of solutions of the system (2.2) as $\alpha \rightarrow 0^{+}$it is crucial to get the uniform in $\alpha$ bounds of various $H^{s}$ norms of the real velocity $V$. Those, however, are not readily feasible. For the uniform in $\alpha$ estimate in $H$ for $V$ we may use the Galerkin procedure as in [22] by estimating the bounds of $H, H^{1}$, and $H^{2}$ norms of $U$, since

$$
|V|^{2}=|U|^{2}+2 \alpha^{2}\|U\|^{2}+\alpha^{4}|A U|^{2} .
$$

We can achieve the uniformness in $\alpha$ only in $H$ and $H^{1}$ for the regularized velocity $U$ but not in $H^{2}$ as we will see below. ${ }^{1}$ Only the $H^{-1}$ norm of $V$ is uniformly bounded in $\alpha$, which is at the root of the difficult problem of finding better uniform estimates.

\footnotetext{
${ }^{1}$ The uniformness of $U$ in $H$ and $H^{1}$ is used to prove the existence of solutions of barotropic limit $\alpha$-equations in Appendix A.
} 
Assume that $F \in H$ with $|F| \leq M_{0, F}$, and $U(0) \in H^{1}$. Let $\left\{w_{j}\right\}_{j=1}^{\infty}$ be an orthonormal basis of $H$ consisting of eigenfunctions of the Stokes operator $A$. Denote $H_{m}=\operatorname{Span}\left\{w_{1}, \ldots, w_{m}\right\}$ and let $P_{m}$ be the $L^{2}$-orthogonal projection from $H$ onto $H_{m} .{ }^{2}$ The Galerkin procedure for Eq. (2.2) (Ch8, [18]) is

$$
\frac{\partial V_{m}}{\partial t}+P_{m} B_{\alpha}\left(V_{m}, V_{m}\right)+\nu A V_{m}+f P_{L} J P_{L} U_{m}=P_{m} F
$$

where $U_{m}=P_{m} U$ and $V_{m}=U_{m}+\alpha^{2} A U_{m}$. Taking the inner product of $(2.5)$ with $U_{m}$ we obtain

$$
\frac{d}{d t}\left\{\left|U_{m}\right|^{2}+\alpha^{2}\left\|U_{m}\right\|^{2}\right\}+\nu\left\{\left\|U_{m}\right\|^{2}+\alpha^{2}\left|A U_{m}\right|^{2}\right\} \leq \frac{1}{\nu} M_{0 F}^{2} .
$$

Using Poincaré and Gronwall's inequalities gives

$$
\left|U_{m}(t)\right|^{2}+\alpha^{2}\left\|U_{m}(t)\right\|^{2} \leq|U(0)|^{2}+\alpha^{2}\|U(0)\|^{2}+\frac{M_{0 F}^{2}}{\nu^{2} \lambda_{1}} \equiv K_{1}, \quad \forall t \geq 0,
$$

where $\lambda_{1}$ is the smallest eigenvalue of Stokes operator $A$ (section 2.1, [37]). Note that $K_{1}$ is an absolute constant, and this estimation is uniform in $\alpha$. By (2.7) $U_{m}$ remains bounded in $L^{\infty}\left(0, T ; H^{1}\right)$, and, for each $m$ and $\alpha>0$, there exists a unique solution $U_{m}$ to the Galerkin system $(2.5)$ on $[0, T]$. In addition, (2.7) implies that

$$
\left|<V_{m}, U_{m}>\right|^{2} \leq K_{1} \quad \text { so that } \quad V_{m} \in L^{\infty}\left(0, T ; H^{-1}\right), \quad \forall m,
$$

where $V_{m}=\left(1+\alpha^{2} A\right) U_{m}$. Integrate (2.6) and use (2.7) to get

$$
\int_{0}^{T}\left\|U_{m}(s)\right\|^{2} d s \leq \frac{K_{1}}{\nu}+\frac{T}{\nu^{2}} M_{0 F}^{2} \equiv k_{1}(T) .
$$

Then $k_{1}(t)<\infty, \forall t \geq 0$, and

$$
U_{m} \in L^{2}\left(0, T ; H^{1}\right)
$$

Notice that the estimate is uniform in $\alpha$; i.e. $k_{1}(t)$ does not depend on $\alpha$. A similar estimate for $V_{m}$ cannot be obtained. Also, from (2.6) and (2.7), we can get

$$
\int_{0}^{t}\left|A U_{m}(s)\right| d s \leq \frac{1}{\alpha^{2}}\left[\frac{t M_{0 F}^{2}}{\nu^{2}}+\frac{K_{1}}{\nu}\right] \equiv \tilde{k}_{1}(t, \alpha) .
$$

This implies that $U_{m} \in L^{2}\left(0, T ; H^{2}\right)$ such that

$$
\left\|U_{m}\right\|_{L^{2}\left(0 . T ; H^{2}\right)}^{2} \leq \tilde{k}_{1}(T, \alpha)
$$

where $\tilde{k}_{1}(t, \alpha)$ satisfies

(i) $\tilde{k}_{1}(t, \alpha)<\infty$ for all $t \geq 0$ and $\alpha>0$,

(ii) $\lim _{t \rightarrow \infty} \tilde{k}_{1}(t, \alpha)=\infty$,

(iii) $\lim _{\alpha \rightarrow 0^{+}} \tilde{k}_{1}(t, \alpha)=\infty$

\footnotetext{
${ }^{2}$ Here $P_{m}$ is not the Leray projector $P_{L}$. This notation is used only in this section. In later sections, $P_{n}$ will denote the $n$-th mode of $P_{L}$ in Fourier space.
} 
We can proceed further to get the $H^{2}$ estimate for $U$ by taking the inner product of Eq. (2.2) with $A U_{m}$ :

$$
\left\|U_{m}(t)\right\|^{2}+\alpha^{2}\left|A U_{m}(t)\right|^{2} \leq k_{2}(t, \alpha) \quad \text { for all } t>0
$$

where $k_{2}(t, \alpha)$ has properties

(a) $k_{2}(t, \alpha)<\infty$ for all $t>0$,

(b) $k_{2}(t, \alpha)$ is independent of $m$,

(c) $k_{2}(t, \alpha)$ depends on $\nu, F$, and $\alpha$; moreover, $\lim _{t \rightarrow 0^{+}} k_{2}(t, \alpha)=\infty$,

(d) $\limsup _{t \rightarrow \infty} k_{2}(t, \alpha)<\infty$,

(e) $\lim _{\alpha \rightarrow 0^{+}} k_{2}(t, \alpha)=\infty$ for every $t>0$.

Part (e) implies that the $H^{2}$-estimate blows up as $\alpha \rightarrow 0^{+}$. A uniform estimate in $\alpha$ is far from trivial. Noticing that $\left|V_{m}\right|^{2}=\left|U_{m}\right|^{2}+2 \alpha^{2}\left\|U_{m}\right\|^{2}+\alpha^{4}\left|A U_{m}\right|^{2}$ where $V_{m}=$ $\left(1+\alpha^{2} A\right) U_{m}$, we obtain

$$
\int_{0}^{t}\left|V_{m}(s)\right|^{2} d s \leq k_{3}(t, \alpha) .
$$

Again, $k_{3}(t, \alpha)$ has similar properties as $k_{2}(t, \alpha)$ does; in particular, it depends on $\alpha$ and blows up as $\alpha \rightarrow 0^{+}$. So we have, $V_{m} \in L^{2}(0, T ; H)$ for finite $\alpha>0$, nonuniformly in $\alpha$. This is at the root of the difficulty in obtaining uniform in $\alpha$ estimates for the unfiltered velocity $V$ for the case of large Coriolis parameter $f>>1$.

The followings are some useful properties on the operator $\mathcal{R}_{\alpha}$ :

Lemma 2.2. In the Fourier space of periodic functions, for each wave number n,

$$
\left(\mathcal{R}_{\alpha}\right)_{n}=\frac{1}{1+\alpha^{2}|\check{n}|^{2}} .
$$

In addition, $\mathcal{R}_{\alpha}$ is commutative with curl.

Lemma 2.3. (Skew-symmetry) $<P_{L} J P_{L} \mathcal{R}_{\alpha} V, A^{\beta} V>=0, \quad \forall \beta \geq 0$.

Proof. By Fourier-Perceval's identity, for every divergence free field $V$,

$$
\begin{aligned}
<P_{L}\left(e_{3} \times \mathcal{R}_{\alpha} V\right), A^{\beta} V> & =\sum_{k \in \mathbb{Z}^{3}}\left(e_{3} \times \frac{V_{k}}{1+\alpha^{2}|\check{k}|^{2}}\right) \cdot|\check{k}|^{2 \beta} \bar{V}_{k} \\
& =\sum_{k>0} \frac{|\check{k}|^{2 \beta}}{1+\alpha^{2}|\check{k}|^{2}}\left[\left(e_{3} \times V_{k}\right) \cdot V_{-k}+\left(e_{3} \times V_{-k}\right) \cdot V_{k}\right] \\
& =0 .
\end{aligned}
$$

3. Resonant limit $\alpha$-equations and operator splitting

Recall the 3D RNS- $\alpha$ equations (2.2):

$$
\begin{gathered}
\frac{\partial V}{\partial t}+f P_{L} J P_{L} \mathcal{R}_{\alpha} V+\nu A V+B_{\alpha}(V, V)=F \\
\nabla \cdot V=0
\end{gathered}
$$

where $B_{\alpha}(V, V)=P_{L}\left[\left(\mathcal{R}_{\alpha} V \cdot \nabla\right) V+V_{j} \nabla\left(\mathcal{R}_{\alpha} V\right)_{j}\right]=-P_{L}\left[\mathcal{R}_{\alpha} V \times \operatorname{curl} V\right]$. In Fourier space, the action $P_{L}$ on $n$-th Fourier component of a vector field is given by 
$P_{L} v=\sum_{n}\left(P_{n} v_{n}\right) e^{i \check{n} \cdot x}$ and $P_{n} v_{n}=\left(v_{n}-\frac{\check{n} \cdot v_{n}}{|\check{n}|^{2}} \check{n}\right)$ with

$$
P_{n}=I-\frac{1}{|\check{n}|^{2}}\left(\begin{array}{ccc}
n_{1}^{2} & \frac{n_{1} n_{2}}{a_{2}} & \frac{n_{1} n_{3}}{a_{3}} \\
\frac{n_{1} n_{2}}{a_{2}} & \frac{n_{2}^{2}}{a_{2}^{2}} & \frac{n_{2} n_{3}}{a_{2} a_{3}} \\
\frac{n_{1} n_{3}}{a_{3}} & \frac{n_{2} n_{3}}{a_{2} a_{3}} & \frac{n_{3}^{2}}{a_{3}^{2}} .
\end{array}\right)
$$

Then, for each wave number $n \in \mathbb{Z}^{3}$, the 3D RNS- $\alpha$ equations have the form

$$
\frac{\partial V_{n}}{\partial t}+\frac{1}{1+\alpha^{2}|\check{n}|^{2}} f P_{n} J P_{n} V_{n}+\nu|\check{n}|^{2} V_{n}+B_{\alpha}(V, V)_{n}=F_{n}
$$

where

$$
B_{\alpha}(V, V)_{n}=-i P_{n} \sum_{k+m=n} \frac{1}{1+\alpha^{2}|\check{k}|^{2}}\left(V_{k} \times\left(\check{m} \times V_{m}\right)\right) .
$$

3.1. Nonlinear averaging. For $\alpha=0$, the linear version of (3.1)-(3.2) was extensively studied by Poincaré $([32])$ and Sobolev ([34]). The Poincaré propagator is the unitary group solution $E_{\alpha}(-f t) \Phi(0)=\Phi(t)\left(E_{\alpha}(0)=I d\right.$ is the identity $)$ to the linear Poincaré problem:

$$
\partial_{t} \Phi+f J \mathcal{R}_{\alpha} \Phi=-\nabla \pi, \quad \nabla \cdot \Phi=0
$$

or, equivalently,

$$
\partial_{t} \Phi+f P_{L} J P_{L} \mathcal{R}_{\alpha} \Phi=0, \quad \nabla \cdot \Phi=0
$$

Denote $M_{\alpha}=P_{L} J P_{L} \mathcal{R}_{\alpha}$ and $M_{\alpha n}=\left(M_{\alpha}\right)_{n}=\frac{1}{1+\alpha^{2}|\check{n}|^{2}} P_{n} J P_{n}$. The matrix $M_{\alpha n}$ has the eigenvalues, $\pm i \xi_{\alpha n}$, where

$$
\xi_{\alpha n}=\frac{\check{n}_{3}}{\left(1+\alpha^{2}|\check{n}|^{2}\right)|\check{n}|}, \quad|\check{n}|=\sqrt{\theta_{1} n_{1}^{2}+\theta_{2} n_{2}^{2}+\theta_{3} n_{3}^{2}}, \quad \theta_{j}=\frac{1}{a_{j}^{2}} .
$$

The Poincaré operator $E_{\alpha}(f t)=e^{f M_{\alpha} t}$ can be written in Fourier space as:

$$
\begin{aligned}
E_{\alpha}(f t)_{n} & =\cos \left(f \xi_{\alpha n} t\right) I+\frac{1}{|\check{n}|} \sin \left(f \xi_{\alpha n} t\right) R_{n}, \\
& =\frac{1}{2}\left[e^{i f \xi_{\alpha n} t}\left(I-i \frac{1}{|\check{n}|} R_{n}\right)+e^{-i f \xi_{\alpha n} t}\left(I+i \frac{1}{|\check{n}|} R_{n}\right)\right],
\end{aligned}
$$

where the matrix $i R_{n}$ is the Fourier transform of the curl vector; $(\operatorname{curl} v)_{n}=i R_{n} v_{n}=$ $i \check{n} \times v_{n}$ with

$$
R_{n}=\left(\begin{array}{ccc}
0 & -\check{n}_{3} & \check{n}_{2} \\
\check{n}_{3} & 0 & -n_{1} \\
-\check{n}_{2} & n_{1} & 0
\end{array}\right)
$$

We introduce the unitary (Van der Pol type) transformation by setting

$$
V=E_{\alpha}(-f t) v(t)
$$


where $v$ is the "slow envelope" variable (also known as a Poincaré variable). Notice that from (3.5) $\xi_{\alpha n}=0$ for $n_{3}=0$ and then, from (3.6), $E_{\alpha}(-f t)$ reduces to the identity operator on any barotropic (vertically averaged) field implying

$$
\begin{aligned}
\bar{V} & =\overline{E_{\alpha}(-f t) v}=\bar{v} \\
\left.V\right|_{t=0} & =\left.v\right|_{t=0} .
\end{aligned}
$$

Since $\frac{\partial V}{\partial t}=-f M_{\alpha} E_{\alpha}(-f t) v+E_{\alpha}(-f t) \frac{\partial v}{\partial t}=-f M_{\alpha} V+E_{\alpha}(-f t) \frac{\partial v}{\partial t}$, the rotational term in Eq. (3.1) is eliminated and Eq. (3.1), in the transformed Poincaré variables, takes the form

$$
\frac{\partial v}{\partial t}+\nu A v=B_{\alpha}(f t, v, v)+E_{\alpha}(f t) F
$$

where

$$
\begin{aligned}
B_{\alpha}(f t, v, v) & =E_{\alpha}(f t) P_{L}\left\{\left[\mathcal{R}_{\alpha} E_{\alpha}(-f t) v\right] \times\left[\operatorname{curl}\left(E_{\alpha}(-f t) v\right)\right]\right\} \\
& =-E_{\alpha}(f t) B_{\alpha}\left(E_{\alpha}(-f t) v, E_{\alpha}(-f t) v\right)
\end{aligned}
$$

The corresponding representation in Fourier space is, for each $n \in \mathbb{Z}^{3}$,

$$
\begin{aligned}
\partial_{t} v_{n} & =B_{\alpha}(f t, v, v)_{n}-\nu|\check{n}|^{2} v_{n}+E_{\alpha}(f t)_{n} F_{n} \\
& =\sum_{k+m=n} B_{\alpha}\left(f t, v_{k}, v_{m}\right)_{n}-\nu|\check{n}|^{2} v_{n}+E_{\alpha}(f t)_{n} F_{n}
\end{aligned}
$$

where

$$
B_{\alpha}\left(f t, v_{k}, v_{m}\right)_{n}=i \frac{1}{1+\alpha^{2}|\check{k}|^{2}} E_{\alpha}(f t)_{n} P_{n}\left[E_{\alpha}(-f t)_{k} v_{k} \times\left(\check{m} \times E_{\alpha}(-f t)_{m} v_{m}\right)\right] .
$$

Eq. (3.10) is explicitly time-dependent with rapidly varying coefficients. This suggests that, for $f>>1$, the dynamic mechanisms of (3.10) evolve over two different time scales; the first one being induced by the fast Poincaré waves and the second given by the evolution of the Poincaré "slow envelope" $v(t)$. The following equations describe reduced dynamics associated with Eq. (3.10) in averaging over fast time scale in the limit $f \rightarrow \infty$ (with $\nu=0$ and $F=0$ ):

$$
\partial_{t} w=\tilde{B}_{\alpha}(w, w), \tilde{B}_{\alpha}(w(t), w(t))=\lim _{f \rightarrow \infty} \frac{1}{2 \pi} \int_{0}^{2 \pi} B_{\alpha}(f s, w(t), w(t)) d s
$$

In Fourier space, the integrand on the right hand side of Eq. (3.12) has terms of the form of $e^{i\left( \pm \xi_{\alpha k} \pm \xi_{\alpha m} \pm \xi_{\alpha n}\right) f t}$, where

$$
\xi_{\alpha k}=\frac{\check{k}_{3}}{|\check{k}|\left(1+\alpha^{2}|\check{k}|^{2}\right)}, \xi_{\alpha m}=\frac{\check{m}_{3}}{|\check{m}|\left(1+\alpha^{2}|\check{m}|^{2}\right)}, \quad \xi_{\alpha n}=\frac{\check{n}_{3}}{|\check{n}|\left(1+\alpha^{2}|\check{n}|^{2}\right)},
$$

with $\check{n}=\check{k}+\check{m}$. Denote $D_{l}(k, m, n)= \pm \xi_{\alpha k} \pm \xi_{\alpha m} \pm \xi_{\alpha n}$, where $l=1,2, \ldots, 8$ is the combination of signs \pm . Then, for $D_{l}(k, m, n) \neq 0$,

$$
\left|\int_{0}^{2 \pi} e^{i\left( \pm \xi_{\alpha k} \pm \xi_{\alpha m} \pm \xi_{\alpha n}\right) f t} d t\right|=\left|\frac{1}{i D_{l}(k, m, n) f} e^{i D_{l}(k, m, n) f t}\right|_{0}^{2 \pi} \mid \leq \frac{2}{f\left|D_{l}(k, m, n)\right|} .
$$


This implies that, for each $n=k+m$, resonances arise as $D_{l}(k, m, n) \rightarrow 0$. That is, we obtain resonant condition for the interactions generated by the quadratic nonlinearity in the limit $f \rightarrow \infty$;

$$
\pm \xi_{\alpha k} \pm \xi_{\alpha m} \pm \xi_{\alpha n}=0 .
$$

Thus $B_{\alpha}(f t, v, v)$ contains resonant terms ( $f t$-independent terms) and nonresonant terms ( $f t$-dependent terms), and we decompose it as

$$
B_{\alpha}(f t, v, v)=\tilde{B}_{\alpha}(v, v)+B_{\alpha}^{o s c}(f t, v, v),
$$

where $B_{\alpha}^{o s c}(f t, v, v)$ contains all nonresonant terms and $\tilde{B}_{\alpha}(v, v)$, "the resonant bilinear operator", contains all resonant terms. Clearly,

$$
B_{\alpha}^{o s c}(f t, v, v)_{n}=\sum_{\substack{l=1,2, \ldots, 8 \\ k+m=n \\ D_{l}(k, m, n) \neq 0}} e^{i f t D_{l}(k, m, n)} Q_{k m n l}\left(v_{k}, v_{m}\right),
$$

where $Q_{k m n l}\left(v_{k}, v_{m}\right)$ is a bilinear form in $v_{k}, v_{m} \in \mathbb{C}^{3}$ with the estimate

$$
\left|Q_{k m n l}\left(v_{k}, v_{m}\right)\right| \leq|\check{m}|\left|v_{k}\right|\left|v_{m}\right| \text {. }
$$

Averaging the nonresonant operator (3.15), which includes oscillating exponential terms with $D_{l}(k, m, n) \neq 0$, yields no contribution to resonant terms in the limit $f \rightarrow \infty$. That is, for each $n$,

$$
\lim _{f \rightarrow \infty} \frac{1}{2 \pi} \int_{0}^{2 \pi} B_{\alpha}^{o s c}(f s, v, v)_{n} d s=0 .
$$

Hence, after averaging (3.8), we arrive at the resonant limit $\alpha$-equations;

$$
\begin{gathered}
\frac{\partial v}{\partial t}+\nu A v=\tilde{B}_{\alpha}(v, v)+\tilde{F} \\
v(0)=V(0)
\end{gathered}
$$

where

$$
\begin{aligned}
\tilde{B}_{\alpha}(v, v) & =\lim _{f \rightarrow \infty} \frac{1}{2 \pi} \int_{0}^{2 \pi} B_{\alpha}(f s, v, v) d s \\
\tilde{F} & =\lim _{f \rightarrow \infty} \frac{1}{2 \pi} \int_{0}^{2 \pi} E_{\alpha}(f s) F d s .
\end{aligned}
$$

We will call $\tilde{B}_{\alpha}(v, v)$ a (bilinear) resonant limit operator.

3.2. Structure of resonant sets. The resonant nonlinear interactions of Poincaré waves for $\tilde{B}(v, v)$ in (3.18) are present when the Poincaré frequencies satisfy the resonant relation $D_{l}(k, m, n)=0$, and we define the corresponding resonant set $K$ by

$$
\begin{aligned}
K & =\left\{(k, m, n) \in \mathbb{Z}^{3}: \pm \xi_{\alpha k} \pm \xi_{\alpha m} \pm \xi_{\alpha n}=0, \quad n=k+m\right\} \\
& =\bigcup_{l=1}^{8} K_{l}
\end{aligned}
$$


where $K_{l}=\left\{(k, m, n): D_{l}(k, m, n)=0, \quad n=k+m\right\}$ and $l$ describes the choice of signs \pm . Define

$$
\begin{array}{cl}
D_{1}(k, m, n)=\xi_{\alpha k}+\xi_{\alpha m}+\xi_{\alpha n}, & D_{2}(k, m, n)=\xi_{\alpha k}-\xi_{\alpha m}+\xi_{\alpha n}, \\
D_{3}(k, m, n)=-\xi_{\alpha k}+\xi_{\alpha m}+\xi_{\alpha n}, & D_{4}(k, m, n)=-\xi_{\alpha k}-\xi_{\alpha m}+\xi_{\alpha n} .
\end{array}
$$

Four other $D_{l}^{\prime} s$ are obtained by multiplication of (3.22) and (3.23) by -1 , and $K$ can be represented as $K=\cup_{l=1}^{4} K_{l}$.

We decompose the resonant set $K$ into three groups for further analysis; pure 2D interactions $\left(K_{2 D}\right)$, two wave interactions $(\tilde{K})$, and three wave interactions $\left(K^{*}\right){ }^{3}$

(i) $K_{2 D}=\left\{(k, m, n) \in K \mid k_{3}=m_{3}=n_{3}=0\right\}$ corresponds to pure two dimensional horizontal interactions (i.e., depends on $x_{1}, x_{2}$ and does not depend on $x_{3}$ in physical space.)

(ii) $\tilde{K}=\left\{(k, m, n) \in K \mid k_{3} m_{3} n_{3}=0, \quad k_{3}^{2}+m_{3}^{2}+n_{3}^{2} \neq 0\right\}$ is the set of two wave resonances. Here $k_{3} m_{3} n_{3}=0$ represents that one or two of $k_{3}, m_{3}$ and $n_{3}$ would be zero. But, if two of them are zero, we have 1-wave interaction which is excluded. It requires the second condition $k_{3}^{2}+m_{3}^{2}+n_{3}^{2} \neq 0$.

This is the case when one of the three frequencies $\xi$ equals zero and two remaining $\xi$ are nonzero;for example, $\left\{(k, m, n) \in K \mid \xi_{\alpha n}=0, \xi_{\alpha k}+\xi_{\alpha m}=0, \xi_{\alpha k} \neq\right.$ $\left.0 \neq \xi_{\alpha m}\right\}=K_{14}=\left(K_{1} \cap K_{4}\right) \backslash K_{2 D} . \tilde{K}$ can be expressed in the way of

$$
\tilde{K}=K_{14} \cup K_{24} \cup K_{34},
$$

where $K_{j 4}=\left(K_{j} \cap K_{4}\right) \backslash K_{2 D}$ for $j=1,2,3$ and

$$
\begin{aligned}
& K_{14}=\left\{(k, m, n) \in K\left|n_{3}=0, \check{k}_{3}=-\check{m}_{3} \neq 0,\right| \check{m}|=| \check{k} \mid\right\} \\
& K_{24}=\left\{k_{3}=0, \check{m}_{3}=\check{n}_{3} \neq 0,|\check{m}|=|\check{n}|\right\} \\
& K_{34}=\left\{m_{3}=0, \check{k}_{3}=\check{n}_{3} \neq 0,|\check{k}|=|\check{n}|\right\} .
\end{aligned}
$$

Formally there exist three more 2 -wave cones, but they are empty sets ([6]).

(iii) $K^{*}=\left\{(k, m, n) \in K \mid k_{3} m_{3} n_{3} \neq 0\right\}$ is the set of strict three wave resonances.

3.3. Explicit formula of the resonant limit operator. The bilinear form $B(f t, v, v)$ in Eq.(3.18) can be written as a sum of even and odd terms in $f t$ :

$$
B_{\alpha}(f t, v, v)=B_{\alpha}^{e v e n}(f t, v, v)+B_{\alpha}^{o d d}(f t, v, v) .
$$

In (3.12), integrating the bilinear operator over $[0,2 \pi]$, the odd terms vanish and make no contribution to resonant terms. Thus we only need to consider the even terms of the operator.

The operator $\exp \left(f M_{\alpha} t\right)$ restricted to divergence free vector fields has the form (3.6) in Fourier space, and acts on Fourier components of divergence free vector fields as

$$
[E(-f t)]_{n} v_{n}=\cos \left(f \xi_{\alpha n} t\right) v_{n}-\frac{1}{|\check{n}|} \sin \left(f \xi_{\alpha n} t\right) R_{n} v_{n}
$$

where $R_{n} v_{n}=\check{n} \times v_{n}$. Denote $C s(f t)$ and $S n(f t)$ by

$$
\begin{aligned}
& {[C s(f t)]_{n}=C s_{n}(f t)=\cos \left(f \xi_{\alpha n} t\right)} \\
& {[S n(f t)]_{n}=S n_{n}(f t)=i \sin \left(f \xi_{\alpha n} t\right) .}
\end{aligned}
$$

\footnotetext{
${ }^{3}$ Note that if two $\xi$ are zero, then using $n_{3}=m_{3}+k_{3}$ we see that the third $\xi$ becomes zero; so 1 -wave interactions are impossible.
} 
Using the fact that the Stokes operator $A=-\Delta$ can be written as $A_{n}=|\check{n}|^{2}$ in Fourier space the equation (3.27) can be expressed as

$$
E(-f t) v=C s(f t) v+A^{-\frac{1}{2}} S n(f t) \operatorname{curl} v
$$

Here we also used $\operatorname{curl}\left(e^{i \check{n} \cdot x} v_{n}\right)=e^{i \check{n} \cdot x} i\left(\check{n} \times v_{n}\right)$. Then, from (3.9), the even terms of $B_{\alpha}(f t, v, v)$ can be written as

$$
B_{\alpha}^{e v e n}(f t, v, v)=\sum_{j=1}^{4} B_{e}^{j}(f t, v, v)
$$

where

$$
\begin{aligned}
& B_{e}^{1}(f t, v, v)=P_{L} C s(f t)\left\{\left(1-\alpha^{2} \Delta\right)^{-1} C s(f t) v \times C s(f t) \operatorname{curl} v\right\} \\
& B_{e}^{2}(f t, v, v)=P_{L} C s(f t)\left\{\left(1-\alpha^{2} \Delta\right)^{-1} A^{-\frac{1}{2}} S n(f t) \operatorname{curl} v \times A^{\frac{1}{2}} S n(f t) v\right\} \\
& B_{e}^{3}(f t, v, v)=-P_{L} A^{-\frac{1}{2}} S n(f t) \operatorname{curl}\left[\left(1-\alpha^{2} \Delta\right)^{-1} C s(f t) v \times A^{\frac{1}{2}} S n(f t) v\right] \\
& B_{e}^{4}(f t, v, v)=-P_{L} A^{-\frac{1}{2}} S n(f t) \operatorname{curl}\left[\left(1-\alpha^{2} \Delta\right)^{-1} A^{-\frac{1}{2}} S n(f t) \operatorname{curl} v \times C s(f t) \operatorname{curl} v\right] .
\end{aligned}
$$

We make an important observation which will produce an operator splitting.

Lemma 3.1. $B_{\alpha}^{\text {even }}\left(f t, v_{k}, v_{m}\right)_{n}$ restricted to $K_{14}$ has no resonant terms.

Proof. Recall that $K_{14}=\left\{(k, m, n) \in K\left|\check{k}_{3}+\check{m}_{3}=0,\right| \check{m}|=| \check{k} \mid\right\}$. Since $\check{n}_{3}=\check{k}_{3}+$ $\check{m}_{3}=0, \sin \left(\xi_{\alpha n} f t\right)=0$ and $\cos \left(\xi_{\alpha n} f t\right)=1$ on $K_{14}$. Thus the bilinear operators $B_{e}^{3}$ and $B_{e}^{4}$ are identically zero on $K_{14}$. On the other hand, the bilinear operators $B_{e}^{1}$ and $B_{e}^{2}$ have the following Fourier coefficients on $K_{14}$ :

$$
\begin{aligned}
& B_{e}^{1}\left(f t, v_{k}, v_{m}\right)_{n}=i \frac{1}{1+\alpha^{2}|\check{k}|^{2}} \cos ^{2}\left(\xi_{\alpha k} f t\right) P_{n}\left[v_{k} \times\left(\check{m} \times v_{m}\right)\right] \\
& B_{e}^{2}\left(f t, v_{k}, v_{m}\right)_{n}=i \frac{1}{1+\alpha^{2}|\check{k}|^{2}} \sin ^{2}\left(\xi_{\alpha k} f t\right) P_{n}\left[\left(\check{k} \times v_{k}\right) \times v_{m}\right] .
\end{aligned}
$$

Then, for each fixed $n$, the operator $B_{\alpha}^{e v e n}(f t, v, v)_{n}$ with interactions restricted to $K_{14}$ takes the form

$$
\begin{aligned}
\left.B_{\alpha}^{e v e n}\left(f t, v_{k}, v_{m}\right)_{n}\right|_{K_{14}} & =i \sum_{(k, m, n) \in K_{14}} \frac{1}{1+\alpha^{2}|\check{k}|^{2}} \cos \left(2 \xi_{\alpha k} f t\right) P_{n}\left[v_{k} \times\left(\check{m} \times v_{m}\right)\right] \\
& =0
\end{aligned}
$$

since the domain of summation $K_{14}$ is symmetric in $k$ and $m, \frac{1}{1+\alpha^{2}|\check{k}|^{2}}=\frac{1}{1+\alpha^{2}|\check{m}|^{2}}$ on $K_{14}$, and $\left(\check{k} \times v_{k}\right) \times v_{m}=-v_{m} \times\left(\check{k} \times v_{k}\right)$. Hence, the lemma is proved.

Also, observe that

$$
\begin{aligned}
& B_{e}^{2}\left(f t, v_{k}, v_{m}\right)_{n}=0 \text { on } K_{24} \text { and } K_{34}, \\
& B_{e}^{3}\left(f t, v_{k}, v_{m}\right)_{n}=0 \text { on } K_{34}, \\
& B_{e}^{4}\left(f t, v_{k}, v_{m}\right)_{n}=0 \text { on } K_{24} .
\end{aligned}
$$


Collecting all the resonant terms gives the following explicit formula:

$$
\begin{aligned}
\left.\tilde{B}_{\alpha}(v, v)_{n}\right|_{K_{2 D} \cup \tilde{K}}= & {\left[\sum_{j=1}^{4} B_{e}^{j}\left(f t, v_{k}, v_{m}\right)_{n}\right]_{K_{2 D} \cup \tilde{K}} } \\
= & i \sum_{K_{2 D}} \frac{1}{1+\alpha^{2}|\check{k}|^{2}} P_{n}\left[v_{k} \times\left(\check{m} \times v_{m}\right)\right] \\
& +\frac{i}{2} \sum_{K_{24} \cup K_{34}} \frac{1}{1+\alpha^{2}|\check{k}|^{2}} P_{n}\left[v_{k} \times\left(\check{m} \times v_{m}\right)\right] \\
& +\frac{i}{2} \sum_{K_{24}} \frac{1}{1+\alpha^{2}|\check{k}|^{2}} P_{n}\left[\check{n} \times\left(v_{k} \times v_{m}\right)\right] \\
& -\frac{i}{2} \sum_{K_{34}} \frac{1}{1+\alpha^{2}|\check{k}|^{2}} \frac{1}{|\check{n}|^{2}} P_{n}\left[\check{n} \times\left\{\left(\check{k} \times v_{k}\right) \times\left(\check{m} \times v_{m}\right)\right\}\right] \\
= & -i \sum_{K_{2 D}} \frac{1}{1+\alpha^{2}|\check{k}|^{2}} P_{n}\left[\left(\check{m} \cdot v_{k}\right) v_{m}\right] \\
& -i \sum_{K_{24}} \frac{1}{1+\alpha^{2}|\check{k}|^{2}} P_{n}\left[\left(\check{m} \cdot v_{k}\right) v_{m}\right] \\
& +\frac{i}{2} \sum_{K_{24}} \frac{1}{1+\alpha^{2}|\check{k}|^{2}} P_{n}\left[\left(v_{k} \cdot v_{m}\right) \check{m}+\left(\check{k} \cdot v_{m}\right) v_{k}\right] \\
& +\frac{i}{2} \sum_{K_{34}} \frac{1}{1+\alpha^{2}|\check{n}|^{2}} P_{n}\left[\left(v_{k} \cdot v_{m}\right) \check{m}-\left(\check{m} \cdot v_{k}\right) v_{m}\right] \\
& -\frac{i}{2} \sum_{K_{34}} \frac{1}{1+\alpha^{2}|\check{n}|^{2}} \frac{1}{|\check{n}|^{2}} P_{n}\left[\check{n} \times\left\{\left(\check{k} \times v_{k}\right) \times\left(\check{m} \times v_{m}\right)\right\}\right] .
\end{aligned}
$$

For the last equality we used the identity $a \times(b \times c)=(a \cdot c) b-(a \cdot b) c$ as well as the fact that $|\breve{k}|^{2}=|\check{n}|^{2}$ on $K_{34}$. Now the bilinear resonant limit operator takes the form

$$
\tilde{B}_{\alpha}(v, v)_{n}=\tilde{B}_{I}^{\alpha}(v, v)_{n}+\tilde{B}_{I I}^{\alpha}(v, v)_{n}+\tilde{B}_{I I I}^{\alpha}(v, v)_{n},
$$

where

$\tilde{B}_{I}^{\alpha}(v, v)=2 \mathrm{D}$ bilinear operator corresponding to the kernel of $P J P S_{\alpha}$

for which $E_{\alpha}(-f t)=I$ ( see Eq. (3.32) for the notation $S_{\alpha}$ )

$=-i \sum_{K_{2 D}} \frac{1}{1+\alpha^{2}|\check{k}|^{2}} P_{n}\left[\left(\check{m} \cdot v_{k}\right) v_{m}\right]$

$\tilde{B}_{I I}^{\alpha}(v, v)=$ Catalytic bilinear operator corresponding to 2-wave resonances

$$
\begin{aligned}
= & -i \sum_{K_{24}} \frac{1}{1+\alpha^{2}|\check{k}|^{2}} P_{n}\left[\left(\check{m} \cdot v_{k}\right) v_{m}\right] \\
& +\frac{i}{2} \sum_{K_{24}} \frac{1}{1+\alpha^{2}|\check{k}|^{2}} P_{n}\left[\left(v_{k} \cdot v_{m}\right) \check{m}+\left(\check{k} \cdot v_{m}\right) v_{k}\right] \\
& +\frac{i}{2} \sum_{K_{34}} \frac{1}{1+\alpha^{2}|\check{n}|^{2}} P_{n}\left[\left(v_{k} \cdot v_{m}\right) \check{m}-\left(\check{m} \cdot v_{k}\right) v_{m}\right] \\
& -\frac{i}{2} \sum_{K_{34}} \frac{1}{1+\alpha^{2}|\check{n}|^{2}} \frac{1}{|\check{n}|^{2}} P_{n}\left[\check{n} \times\left\{\left(\check{k} \times v_{k}\right) \times\left(\check{m} \times v_{m}\right)\right\}\right]
\end{aligned}
$$

$\tilde{B}_{I I I}^{\alpha}(v, v)=$ Resonant bilinear operator corresponding to 3 -wave resonances

$$
=\sum_{(k, m, n) \in K^{*}} Q_{k m n}\left(v_{k}, v_{m}\right)
$$


3.4. Barotropic and Baroclinic projections: operator splitting. Let $\bar{V}$ be the $x_{3}$-averaging of $V \in H$ :

$$
\bar{V}\left(t, x_{1}, x_{2}\right)=\frac{1}{2 \pi a_{3}} \int_{0}^{2 \pi a_{3}} V\left(t, x_{1}, x_{2}, x_{3}\right) d x_{3} .
$$

Denote $\mathcal{M}=\left\{\bar{V}\left(t, x_{1}, x_{2}\right) \mid V \in H\right\}$. Then $\mathcal{M}$ is a closed subspace of $H$, and any $V \in$ $H$ has a unique representation $V=\bar{V}+V^{\perp}$ with $\overline{V^{\perp}}=0$. We define an orthogonal projection $P_{b}: H \rightarrow H$ by

$$
P_{b} V=\bar{V}=\frac{1}{2 \pi a_{3}} \int_{0}^{2 \pi a_{3}} V\left(t, x_{1}, x_{2}, x_{3}\right) d x_{3} .
$$

We call $P_{b}$ a barotropic projection. The corresponding projection $P_{b}^{\perp}: H \rightarrow H$ defined by

$$
P_{b}^{\perp} V=V^{\perp}
$$

is called a baroclinic projection. With $\bar{H}=P_{b} H$ and $\overline{H^{\perp}}=P_{b}^{\perp} H$ we obtain an orthogonal decomposition $H=\bar{H} \oplus H^{\perp}$.

The barotropic projection is the projection on the null space of $P_{L} J P_{L} S_{\alpha}$, where $S_{\alpha}$ is the matrix of filtering operator and, in Fourier space, it has the form

$$
S_{\alpha n}=\left[\begin{array}{ccc}
\frac{1}{1+\alpha^{2}|\check{n}|^{2}} & 0 & 0 \\
0 & \frac{1}{1+\alpha^{2}|\breve{n}|^{2}} & 0 \\
0 & 0 & \frac{1}{1+\alpha^{2}|\check{n}|^{2}}
\end{array}\right] .
$$

This is the infinite-dimensional space of divergence free fields which depends on only $x_{1}, x_{2}$. Hence $E_{\alpha}(-f t) P_{b} \equiv I$.

Lemma 3.2. In Fourier space, the averaging in $x_{3}$ is equivalent to $n_{3}=0$. That is, for each wave number $n, \bar{V}$ is the restriction of $V$ to $n_{3}=0$.

This Lemma together with resonant sets in section 3.3 induces the following representation of the resonant limit operator in (3.18):

$$
\begin{aligned}
\tilde{B}_{\alpha}(w, w) & =\tilde{B}_{\alpha}(\bar{w}, \bar{w})+\tilde{B}_{\alpha}\left(\bar{w}, w^{\perp}\right)+\tilde{B}_{\alpha}\left(w^{\perp}, \bar{w}\right)+\tilde{B}_{\alpha}\left(w^{\perp}, w^{\perp}\right) \\
& =\tilde{B}_{I}^{\alpha}(\bar{w}, \bar{w})+\tilde{B}_{I I}^{\alpha}\left(\bar{w}, w^{\perp}\right)+\tilde{B}_{I I I}^{\alpha}\left(w^{\perp}, w^{\perp}\right)
\end{aligned}
$$

where

$$
\begin{aligned}
\tilde{B}_{I}^{\alpha}(\bar{w}, \bar{w}) & =i \sum_{K_{2 D}} \frac{1}{1+\alpha^{2}|\check{k}|^{2}} P_{n}\left[\bar{w}_{k} \times\left(\check{m} \times \bar{w}_{m}\right)\right] \\
& =-i \sum_{K_{2 D}} \frac{1}{1+\alpha^{2}|\check{k}|^{2}} P_{n}\left[\left(\check{m} \cdot \bar{w}_{k}\right) \bar{w}_{m}\right]
\end{aligned}
$$




$$
\begin{aligned}
\tilde{B}_{I I}^{\alpha}\left(\bar{w}, w^{\perp}\right)= & -i \sum_{K_{24}} \frac{1}{1+\alpha^{2}|\check{k}|^{2}} P_{n}\left[\left(\check{m} \cdot \bar{w}_{k}\right) w_{m}^{\perp}\right] \\
& +\frac{i}{2} \sum_{K_{24}} \frac{1}{1+\alpha^{2}|\check{k}|^{2}} P_{n}\left[\left(\bar{w}_{k} \cdot w_{m}^{\perp}\right) \check{m}+\left(\check{k} \cdot w_{m}^{\perp}\right) \bar{w}_{k}\right] \\
& +\frac{i}{2} \sum_{K_{34}} \frac{1}{1+\alpha^{2}|\check{n}|^{2}} P_{n}\left[\left(w_{k}^{\perp} \cdot \bar{w}_{m}\right) \check{m}-\left(\check{m} \cdot w_{k}^{\perp}\right) \bar{w}_{m}\right] \\
& -\frac{i}{2} \sum_{K_{34}} \frac{1}{1+\alpha^{2}|\check{n}|^{2}} \frac{1}{|\check{n}|^{2}} P_{n}\left[\check{n} \times\left\{\left(\check{k} \times w_{k}^{\perp}\right) \times\left(\check{m} \times \bar{w}_{m}\right)\right\}\right] \\
\tilde{B}_{I I I}^{\alpha}\left(w^{\perp}, w^{\perp}\right)= & \sum_{(k, m, n) \in K^{*}} Q_{k m n}\left(w_{k}^{\perp}, w_{m}^{\perp}\right) .
\end{aligned}
$$

Now, we obtain the same important result as the one in ([6]):

LEMma 3.3. The barotropic projection commutes with the bilinear limit operator; that is,

$$
\overline{\tilde{B}_{\alpha}(w, w)}=\tilde{B}_{\alpha}(\bar{w}, \bar{w})=\tilde{B}_{I}^{\alpha}(\bar{w}, \bar{w}) .
$$

Proof. The result follows from Lemma 3.1 and Lemma 3.2 on the structure of resonant sets (3.24), (3.25), (3.26) and $K^{*}$ as well as (3.28), (3.28) and (3.28).

Lemma 3.3 immediately implies the following properties for $P_{b}$ acting on the two and three wave bilinear limit operators;

LEMMA 3.4. The barotropic projector annihilates the catalytic and three wave resonant limit operators:

(a) $P_{b} \tilde{B}_{I I}^{\alpha}\left(\bar{w}, w^{\perp}\right)=0$

(b) $P_{b} \tilde{B}_{I I I}^{\alpha}\left(w^{\perp}, w^{\perp}\right)=0$

Recalling (3.16) of the form

$$
\begin{aligned}
& \frac{\partial w}{\partial t}+\nu A w=\tilde{B}_{\alpha}(w, w)+\tilde{F} \\
& w(0)=v(0)=V(0), \quad \nabla \cdot w=0
\end{aligned}
$$

where $F, w(0) \in V$, we obtain the following:

Proposition 3.5. (Operator Splitting) Equation (3.43) and (3.44) split into the triangular systems;

(a)

$$
\begin{aligned}
& \frac{\partial \bar{w}}{\partial t}+\nu A \bar{w}=\tilde{B}_{I}^{\alpha}(\bar{w}, \bar{w})+\bar{F} \\
& \bar{w}(0)=\bar{v}(0)=\bar{V}(0), \quad \nabla \cdot \bar{w}=0 .
\end{aligned}
$$

This system is called the barotropic (resonant) limit $\alpha$-equations, which is identical to the two dimensional and three-component Navier-Stokes- $\alpha$ equations. 
(b)

$$
\begin{gathered}
\frac{\partial w^{\perp}}{\partial t}+\nu A w^{\perp}=\tilde{B}_{I I}^{\alpha}\left(\bar{w}, w^{\perp}\right)+\tilde{B}_{I I I}^{\alpha}\left(w^{\perp}, w^{\perp}\right)+\tilde{F}^{\perp} \\
w^{\perp}(0)=v^{\perp}(0)=V^{\perp}(0), \quad \nabla \cdot w^{\perp}=0 .
\end{gathered}
$$

This system is called the baroclinic (resonant) limit $\alpha$-equations.

Proof. Take $P_{b}$ on (3.43) and (3.44) and use the followings:

(a) $0=P_{b}(\nabla \cdot w)=\nabla \cdot P_{b} w=\nabla \cdot \bar{w}$.

(b) $0=\nabla \cdot w=\nabla \cdot \bar{w}+\nabla \cdot w^{\perp}$. Since $\nabla \cdot \bar{w}=0, \nabla \cdot w^{\perp}=0$.

\section{Existence and regularity of limit $\alpha$-equations}

In this section, our main theme is the existence and global regularity of solutions of resonant limit $\alpha$-equations with estimates independent of the parameter $\alpha$. Independence from $\alpha$ is at the core in this paper, which will establish the uniform convergence of RNS- $\alpha$ equations as $\alpha \rightarrow 0$.

4.1. Barotropic limit $\alpha$-equations. The barotropic limit $\alpha$-equations of RNS- $\alpha$ equations are

$$
\begin{aligned}
\frac{\partial \bar{w}}{\partial t}+\nu A \bar{w} & =\tilde{B}_{I}^{\alpha}(\bar{w}, \bar{w})+\bar{F} \\
\bar{w}\left(0, x_{1}, x_{2}\right) & =\bar{w}(0), \quad \nabla \cdot \bar{w}=0
\end{aligned}
$$

where $\tilde{B}_{I}^{\alpha}(\bar{w}, \bar{w})=P_{L}\left[\mathcal{R}_{\alpha} \bar{w} \times \operatorname{curl} \bar{w}\right]$. We denote the horizontal velocity vector field by $\bar{w}_{h}=\left(\bar{w}_{1}\left(t, x_{1}, x_{2}\right), \bar{w}_{2}\left(t, x_{1}, x_{2}\right), 0\right)$ and we understand $\bar{w}_{3}=\left(0,0, \bar{w}_{3}\left(t, x_{1}, x_{2}\right)\right)$ as the vertical velocity vector field component. Then the nonlinear term can be written as

$$
\begin{aligned}
\mathcal{R}_{\alpha} \bar{w} \times \operatorname{curl} \bar{w}= & \left(\mathcal{R}_{\alpha} \bar{w}_{h}+\mathcal{R}_{\alpha} \bar{w}_{3}\right) \times\left\{\operatorname{curl}\left(\bar{w}_{h}+\bar{w}_{3}\right)\right\} \\
= & {\left[\mathcal{R}_{\alpha} \bar{w}_{h} \times \operatorname{curl} \bar{w}_{h}\right]+\left[\mathcal{R}_{\alpha} \bar{w}_{h} \times \operatorname{curl} \bar{w}_{3}\right] } \\
& +\left[\mathcal{R}_{\alpha} \bar{w}_{3} \times \operatorname{curl} \bar{w}_{h}\right]+\left[\mathcal{R}_{\alpha} \bar{w}_{3} \times \operatorname{curl} \bar{w}_{3}\right] .
\end{aligned}
$$

The third term vanishes. The second term represents vertical modes, and the first and fourth consist of horizontal modes. Then the equation (4.1), in the component form, splits as:

$$
\begin{aligned}
& \frac{\partial \bar{w}_{h}}{\partial t}+\nu A \bar{w}_{h}=P_{L}\left[\left(\mathcal{R}_{\alpha} \bar{w}_{h} \times \operatorname{curl} \bar{w}_{h}\right)+\left(\mathcal{R}_{\alpha} \bar{w}_{3} \times \operatorname{curl} \bar{w}_{3}\right)\right]+\bar{F}_{h} \\
& \frac{\partial \bar{w}_{3}}{\partial t}+\nu A \bar{w}_{3}=P_{L}\left[\mathcal{R}_{\alpha} \bar{w}_{h} \times \operatorname{curl} \bar{w}_{3}\right]+\bar{F}_{3} .
\end{aligned}
$$

Note that the barotropic limit equations of rotating NSE are completely decoupled into horizontal and vertical components (see [6] and [7]) but rotating NS- $\alpha$ equations have a coupled 2D3C (two dimensional and three component: dependence on two variables $\left.x_{1}, x_{2}\right)$ system (4.3) and (4.4).

Following the standard way like for the classical 2D NSE we can obtain the unique existence of a solution of the barotropic limit $\alpha$-equations. There, however, is a difference between the $\alpha$-equations and the usual 2D NSE. The bilinear operator $\tilde{B}_{I}^{\alpha}$ doesn't have the usual orthogonal property as the 2D NSE does; $\left\langle\tilde{B}_{I}^{\alpha}(w, w), A^{\beta} w\right\rangle \neq$ 0 for any $w \in V$ and $\beta \geq 0$. But, it has a certain orthogonal property;

$$
<\tilde{B}_{I}^{\alpha}(w, w), A^{\beta} \mathcal{R}_{\alpha} w>=0 \text { for any } w \in V .
$$


TheOrem 4.1. Let $T>0$ be given. Assume that $\bar{F}$ is a time-independent force such that

$$
|\bar{F}|^{2} \leq M_{0 F}^{2},
$$

and let $\bar{w}(0) \in H^{1}$. Then the barotropic limit equations (4.1) and (4.2) have a unique regular solution $\bar{w}$ on $[0, T]$ that satisfies $\bar{w} \in L^{\infty}\left(0, T ; H^{1}\right) \cap L^{2}\left(0, T ; H^{2}\right)$. Moreover, the estimates are uniform in $\alpha$.

The proof of Theorem 4.1 is provided in Appendix A.

4.2. Catalytic limit $\alpha$-equations. In this section we consider the baroclinic limit $\alpha$-equations (3.47) NOT including the three-wave resonant operator $\tilde{B}_{I I I}^{\alpha}$. The latter is only present for $1 / a_{2}, 1 / a_{3}$ of Lebesque measure zero ([6], [7]). The resulting system is called the catalytic limit $\alpha$-equations:

$$
\begin{aligned}
& \frac{\partial w^{\perp}}{\partial t}+\nu A w^{\perp}=\tilde{B}_{I I}^{\alpha}\left(\bar{w}, w^{\perp}\right)+\tilde{F}^{\perp} \\
& w^{\perp}(0)=w_{0}^{\perp}, \quad \nabla \cdot w^{\perp}=0 .
\end{aligned}
$$

Recalling Lemma 3.2, the vector field $\bar{w}$ has the form of Fourier coefficients $\bar{w}_{k}(t)=$ $\bar{w}_{\left(k_{h}, 0\right)}(t)$, where $k_{h}=\left(k_{1}, k_{2}\right) \in \mathbb{Z}^{2}$. We, from [6], adopt the definition

$$
\|\bar{w}\|_{H_{\infty}^{0}}=\sup _{k_{h} \in \mathbb{Z}^{2}}\left|\bar{w}_{k}\right|^{2} .
$$

Since $\quad|\bar{w}|^{2}=\sum_{k_{h}}\left|\bar{w}_{k_{h}}\right|^{2} \geq \sup _{k_{h}}\left|\bar{w}_{k}\right|^{2}, \quad \|\left.\bar{w}\right|_{H_{\infty}^{0}} \leq|\bar{w}| . \quad$ In addition, $\quad \|\left.\bar{w}\right|_{H_{\infty}^{0}} \leq$ $\left(a_{1} a_{2}\right)^{-1}(2 \pi)^{-2}|| \bar{w} \|_{L^{1}}$. We follow the BMN's procedure (Lemma 5.1 and Theorem 5.3 in [6]) to establish the regularity, commutativity, and skew-symmetry properties of catalytic bilinear operator (the proof of Theorem 4.2 is given in Appendix B):

Theorem 4.2. Let $\bar{w} \in H$ and $w^{\perp} \in H^{2 s+1}, s \geq 0$. Then

1. $\tilde{B}_{I L}^{\alpha}\left(\bar{w}, A^{s} w^{\perp}\right)=A^{s} \tilde{B}_{I I}^{\alpha}\left(\bar{w}, w^{\perp}\right)$.

2. $<B_{I I}^{\alpha}\left(\bar{w}, w^{\perp}\right), A^{s} w^{\perp}>=0$

3. $\tilde{B}_{I L}^{\alpha}\left(\bar{w}, \partial_{3}^{l} w^{\perp}\right)=\partial_{3}^{l} \tilde{B}_{I I}^{\alpha}\left(\bar{w}, w^{\perp}\right)$.

4. $<B_{I I}^{\alpha}\left(\bar{w}, w^{\perp}\right), \partial_{3}^{l} w^{\perp}>=0$.

5. $\left\|\tilde{B}_{I I}^{\alpha}\left(\bar{w}, w^{\perp}\right)\right\|_{s} \leq 8 \sqrt{2}\|\bar{w}\|_{H_{\infty}^{0}}\left\|w^{\perp}\right\|_{s+1} \leq 8 \sqrt{2}|\bar{w}|\left\|w^{\perp}\right\|_{s+1}$.

The existence and uniqueness of regular solution of catalytic limit $\alpha$-equations can be easily obtained using Theorem 4.2 :

THEOREM 4.3. Let $F^{\perp}$ be a force with $\left|F^{\perp}\right|^{2} \leq M_{0 f}$, and let $w^{\perp}(0) \in H^{1}$. Then, for any time $T>0$, the catalytic limit equations (4.6) and (4.7) have a unique regular solution in $L^{\infty}\left(0, T ; H^{1}\right) \cap L^{2}\left(0, T ; H^{2}\right)$. Moreover, the estimates are uniform in $\alpha$.

Proof. By the property 2 of Theorem 4.2, we have

$$
<\tilde{B}_{I I}^{\alpha}\left(\bar{w}, w^{\perp}\right), A^{s} w^{\perp}>=0, \quad \forall s \geq 0 .
$$

- $L^{2}$-estimate of $w^{\perp}$ : Taking an inner product (4.6) with $w^{\perp}$ yields

$$
\frac{1}{2} \frac{d}{d t}\left|w^{\perp}\right|^{2}+\nu\left\|w^{\perp}\right\|^{2} \leq \frac{\nu}{2}\left|w^{\perp}\right|^{2}+\frac{1}{2 \nu}\left|F^{\perp}\right|^{2} .
$$

Let $y=\left|w^{\perp}\right|^{2}$. Then we obtain

$$
\frac{d y}{d t} \leq g y+h,
$$


where $g=\nu$ and $h=\frac{1}{\nu}\left|F^{\perp}\right|^{2}$. By the classical Gronwall's inequality, we obtain

$$
y(t) \leq\left[y(0)+\frac{M_{0 F}^{2}}{\nu^{2}}\right] e^{\nu T}, \quad \forall t \in[0, T] .
$$

Thus

$$
\left|w^{\perp}(t)\right|^{2} \leq\left[\left|w^{\perp}(0)\right|^{2}+\frac{M_{0 F}^{2}}{\nu}\right] e^{\nu T} \equiv\left(\rho_{H}^{\perp}(T)\right)^{2}, \quad \forall t \in[0, T],
$$

and

$$
w^{\perp} \in L^{\infty}(0, T ; H), \quad \forall T>0 .
$$

Furthermore, from (4.8) and (4.9),

$$
\int_{0}^{T}\left\|w^{\perp}\right\|^{2} d s \leq \frac{1}{2}\left(\rho_{H}^{\perp}(T)\right)^{2}+\frac{M_{0 F}^{2} T}{2 \nu^{2}} \equiv\left(M_{H^{1}}^{\perp}(T)\right)^{2},
$$

and hence,

$$
w^{\perp} \in L^{2}\left(0, T ; H^{1}\right), \quad \forall T>0 .
$$

- $H^{1}$-estimate of $w^{\perp}$ : Take an inner product (4.6) with $A w^{\perp}$ to get

$$
\frac{d}{d t}\left\|w^{\perp}\right\|^{2}+\nu\left|A w^{\perp}\right|^{2} \leq \frac{1}{\nu}\left|F^{\perp}\right|^{2} .
$$

Using Poincaré inequality again, we get

$$
\frac{d}{d t}\left\|w^{\perp}\right\|^{2}+\lambda_{1} \nu\left\|w^{\perp}\right\|^{2} \leq \frac{1}{\nu}\left|F^{\perp}\right|^{2} .
$$

Let $y=\left\|w^{\perp}\right\|^{2}$. Then

$$
\frac{d y}{d t} \leq g y+h,
$$

where $g=-\lambda_{1} \nu$ and $h=\frac{1}{\nu}\left|F^{\perp}\right|^{2}$. The Gronwall's inequality gives

$$
y(t) \leq y(0)+\frac{M_{0 F}^{2}}{\lambda_{1} \nu^{2}} \equiv\left(\rho_{H^{1}}^{\perp}\right)^{2} .
$$

This implies

$$
w^{\perp} \in L^{\infty}\left(0, T ; H^{1}\right), \quad \forall T>0 .
$$

From (4.11) and (4.12) we obtain

$$
\int_{0}^{T}\left|A w^{\perp}(s)\right|^{2} d s \leq \frac{M_{0 F}^{2} T}{\nu^{2}}+\frac{\left(\rho_{H^{1}}\right)^{2}}{\nu} \equiv\left(M_{A}^{\perp}(T)\right)^{2},
$$

and hence

$$
w^{\perp} \in L^{2}\left(0, T ; H^{2}\right), \quad \forall T>0 .
$$


4.3. Baroclinic limit $\alpha$-equations. In this section, we prove the existence of a unique regular solution of the baroclinic limit $\alpha$-equations (3.47) and (3.48) which include the 3 -wave resonant interactions. As usual, we need to establish the regularity result on the baroclinic bilinear operator $\tilde{B}_{I I I}^{\alpha}$. To do that we will follow the procedure developed in [7].

We start off by stating the following lemma whose proof is exactly the same as the one in Lemma 2.1, [7].

Lemma 4.4. Let $(u, v, w) \in H^{\frac{3}{4}} \times H^{\frac{3}{4}} \times H^{1}$. Then

$$
<\tilde{B}_{\alpha}(u, v), w>=\lim _{f \rightarrow \infty} \frac{1}{2 \pi} \int_{0}^{2 \pi}<B_{\alpha}(f s, u, v), w>d s .
$$

The bilinear operator B in the classical NSE enjoys the identity $\langle B(w, w), A w\rangle$ $=-<B(\operatorname{curl} w, w), \operatorname{curl} w>$ for any $w \in H^{2}$. We investigate whether the operator $B_{\alpha}$, $B_{\alpha}(u, v)=-P_{L}\left\{\mathcal{R}_{\alpha} u \times \operatorname{curl} v\right\}$, have the same property. It turns out that it has a similar identity (but not exactly the same).

Lemma 4.5. Let $w \in H^{2}$. The operator $B_{\alpha}$ satisfies the identity

$$
\begin{aligned}
<B_{\alpha}(w, w), A w> & =<(\operatorname{curl} w \cdot \nabla) \operatorname{curl} w, \mathcal{R}_{\alpha} w> \\
& =-<(\operatorname{curl} w \cdot \nabla) \mathcal{R}_{\alpha} w, \operatorname{curl} w>.
\end{aligned}
$$

Proof.

$$
\begin{aligned}
<B_{\alpha}(w, w), A w>= & -<\mathcal{R}_{\alpha} w \times \operatorname{curl} w, \operatorname{curl}^{2} w> \\
=-< & \operatorname{curl}\left[\mathcal{R}_{\alpha} w \times \operatorname{curl} w\right], \operatorname{curl} w> \\
=<-(\operatorname{curl} w \cdot \nabla) \mathcal{R}_{\alpha} w, \operatorname{curl} w> & \quad+<\left(\mathcal{R}_{\alpha} w \cdot \nabla\right) \operatorname{curl} w, \operatorname{curl} w>.
\end{aligned}
$$

For the third equality we used the identity $\operatorname{curl}(a \times b)=a \operatorname{div} b-b \operatorname{div} a+(b \cdot \nabla) a-(a$. $\nabla) b$ to get

$$
\begin{aligned}
\operatorname{curl}\left[\mathcal{R}_{\alpha} w \times \operatorname{curl} w\right]= & \mathcal{R}_{\alpha} w \operatorname{div}(\operatorname{curl} w)-\operatorname{curl} w \operatorname{div}\left(\mathcal{R}_{\alpha} w\right) \\
& +(\operatorname{curl} w \cdot \nabla) \mathcal{R}_{\alpha} w-\left(\mathcal{R}_{\alpha} w \cdot \nabla\right) \operatorname{curl} w .
\end{aligned}
$$

Notice that the first and second terms vanish.

Since $<\left(\mathcal{R}_{\alpha} w \cdot \nabla\right) \operatorname{curl} w, \operatorname{curl} w>=0$, we get

$$
<B_{\alpha}(w, w), A w>=<-(\operatorname{curl} w \cdot \nabla) \mathcal{R}_{\alpha} w, \operatorname{curl} w>.
$$

Notice that, for any $u, v \operatorname{such}$ that $\operatorname{div} u=\operatorname{div} v=0$,

$$
-(v \cdot \nabla) u=v \times \operatorname{curl} u-v_{j} \nabla u_{j} .
$$

By setting $v=\operatorname{curl} w$ and $u=\mathcal{R}_{\alpha} w$, we obtain

$$
-(\operatorname{curl} w \cdot \nabla) \mathcal{R}_{\alpha} w=\operatorname{curl} w \times \operatorname{curl}\left(\mathcal{R}_{\alpha} w\right)-(\operatorname{curl} w)_{j} \nabla\left(\mathcal{R}_{\alpha} w\right)_{j} .
$$

Substitution of this into Eq. (4.14) and the equality $<\operatorname{curl} w \times \operatorname{curl}\left(\mathcal{R}_{\alpha} w\right), \operatorname{curl} w>=0$ yield 


$$
\begin{aligned}
<B_{\alpha}(w, w), A w> & =-<(\operatorname{curl} w)_{j} \nabla\left(\mathcal{R}_{\alpha} w\right)_{j}, \operatorname{curl} w> \\
& =-\int(\operatorname{curl} w)_{j} \frac{\partial}{\partial x_{i}}\left(\mathcal{R}_{\alpha} w\right)_{j}(\operatorname{curl} w)_{i} d x \\
& =\int(\operatorname{curl} w)_{i} \frac{\partial}{\partial x_{i}}(\operatorname{curl} w)_{j}\left(\mathcal{R}_{\alpha} w\right)_{j} d x \\
& =\int(\operatorname{curl} w \cdot \nabla) \operatorname{curl} w \cdot \mathcal{R}_{\alpha} w d x .
\end{aligned}
$$

From Lemma 4.4 and Lemma 4.5 we can obtain the following identity by passing to the limit in averaging:

Corollary 4.6. Let $w \in H^{2}$. The resonant limit operator $\tilde{B}_{\alpha}$ satisfies the identity

$$
<\tilde{B}_{\alpha}(w, w), A w>=-<(\operatorname{curl} w \cdot \nabla) \mathcal{R}_{\alpha} w, \operatorname{curl} w>.
$$

The Corollary 4.6 induces the following inequality for the 3 -wave resonant operator:

$$
\begin{aligned}
\left|<\tilde{B}_{I I I}^{\alpha}\left(w^{\perp}, w^{\perp}\right), A w^{\perp}>\right|=\left|<\left(\operatorname{curl} w^{\perp} \cdot \nabla\right) \mathcal{R}_{\alpha} w^{\perp}, \operatorname{curl} w^{\perp}>\right| \\
\quad \leq c \sum_{k+m+n=0}|\check{k}|\left|w_{k}^{\perp}\right||\check{m}|\left|\left(\mathcal{R}_{\alpha} w^{\perp}\right)_{m}\right||\check{n}|\left|w_{n}^{\perp}\right| \mathcal{X}(k, m, n) \\
\leq c \sum_{k+m+n=0}|\check{k}|\left|w_{k}^{\perp}\right||\check{m}|\left|w_{m}^{\perp}\right||\check{n}|\left|w_{n}^{\perp}\right| \mathcal{X}(k, m, n) .
\end{aligned}
$$

For the last inequality we used $\left(\mathcal{R}_{\alpha} w^{\perp}\right)_{m}=\frac{1}{1+\alpha^{2}|\check{m}|^{2}} w_{m}^{\perp}$ so that $\left|\left(\mathcal{R}_{\alpha} w^{\perp}\right)_{m}\right| \leq\left|w_{m}^{\perp}\right|$. Here, $\mathcal{X}(k, m,-n)$ is the characteristic function of the resonant set $K^{*}$ of strict threewave resonances:

$$
\pm \xi_{\alpha k} \pm \xi_{\alpha m} \pm \xi_{\alpha n}=0, \quad n+k+m=0, \quad k_{3} m_{3} n_{3} \neq 0,
$$

where $\xi_{\alpha k}=\frac{\check{k}_{3}}{\left(1+\alpha^{2}|\vec{k}|^{2}\right)|\grave{k}|}$ and similarly for $\xi_{\alpha m}, \xi_{\alpha n}$. This set lies in the set of solutions of the equation

$$
\begin{array}{r}
P(k, m, n)=|\check{k}|^{4}|\check{m}|^{4}|\check{n}|^{4}\left(1+\alpha^{2}|\check{k}|^{2}\right)^{4}\left(1+\alpha^{2}|\check{m}|^{2}\right)^{4}\left(1+\alpha^{2}|\check{n}|^{2}\right)^{4} \\
{\left[\left(\xi_{\alpha k}^{2}+\xi_{\alpha m}^{2}+\xi_{\alpha n}^{2}\right)^{2}-4 \xi_{\alpha k}^{2} \xi_{\alpha m}^{2}\right]=0 .}
\end{array}
$$

Recall that $|\check{k}|^{2}=\theta_{1} k_{1}^{2}+\theta_{2} k_{2}^{2}+\theta_{3} k_{3}^{2}, \theta_{j}=\frac{1}{a_{j}}(j=1,2,3)$, and similarly for $\check{m}, \check{n}$. This equation can be obtained from considering the product of all $D_{l}(k, m, n)$ in $(3.22)$ and (3.23) (this product is zero on the resonant set $K$ );

$$
\begin{aligned}
\left(\xi_{\alpha k}+\xi_{\alpha m}+\xi_{\alpha n}\right)\left(\xi_{\alpha k}-\xi_{\alpha m}+\xi_{\alpha n}\right)\left(\xi_{\alpha k}-\xi_{\alpha m}-\xi_{\alpha n}\right)\left(\xi_{\alpha k}+\xi_{\alpha m}-\xi_{\alpha n}\right) & \\
= & {\left[\left(\xi_{\alpha k}^{2}+\xi_{\alpha m}^{2}+\xi_{\alpha n}^{2}\right)^{2}-4 \xi_{\alpha k}^{2} \xi_{\alpha m}^{2}\right] }
\end{aligned}
$$

Without loss of generality we put $\theta_{1}=\theta_{2}=\theta_{3}=1$.

The equation $P$ is a polynomial in $k, m, n$, and it has the symmetric property: 
Lemma 4.7. The polynomial $P$ is symmetric with respect to $k, m$, and $n$;

$$
P(k, m, n)=P(m, k, n)=P(k, n, m)=P(m, n, k) .
$$

Proof. It is sufficient to consider the factorization (4.16). Changing $k$ to $m, m$ to $n$, and $n$ to $k,(4.16)$ becomes

$$
\begin{aligned}
&\left(\xi_{\alpha k}+\xi_{\alpha m}+\xi_{\alpha n}\right)\left(\xi_{\alpha k}-\xi_{\alpha m}+\xi_{\alpha n}\right)\left(\xi_{\alpha k}-\xi_{\alpha m}-\xi_{\alpha n}\right)\left(\xi_{\alpha k}+\xi_{\alpha m}-\xi_{\alpha n}\right) \\
&=\left(\xi_{\alpha k}+\xi_{\alpha m}+\xi_{\alpha n}\right)\left(\xi_{\alpha k}+\xi_{\alpha m}-\xi_{\alpha n}\right) \\
&\left\{(-1)\left(\xi_{\alpha k}-\xi_{\alpha m}+\xi_{\alpha n}\right)\right\}\left\{(-1)\left(\xi_{\alpha k}-\xi_{\alpha m}-\xi_{\alpha n}\right)\right\} .
\end{aligned}
$$

Compared to (4.16) this contains exactly the same components as those in (4.16). $\mathrm{a}$

By this lemma $\mathcal{X}(k, m, n)$ is equipped with symmetry, and we can take advantage of Lemma 3.1 of [7]. For the detailed proof, see [7]:

Lemma 4.8. (Restricted Convolution Lemma) Let $\mathcal{X}(k, m, n)$ be the characteristic function of some resonant set $K^{*}$ in $\left(\mathbb{Z}^{3}\right)^{3}$ such that $\mathcal{X}(k, m, n)=\mathcal{X}(m, k, n)=$ $\mathcal{X}(k, n, m)$ is symmetric. Let $\gamma \geq 0, \beta$ fixed and

$$
\sup _{n} \sum_{k: k+m+n=0, k \in \sum_{i}} \mathcal{X}(k, m, n)|k|^{-\gamma} \leq C_{0} 2^{i \beta}
$$

for every $i=0,1,2, \ldots$ where

$$
\sum_{i}=\left\{k=\left(k_{1}, k_{2}, k_{3}\right)\left|2^{i} \leq\right| k\left|<2^{i+1},\right| k \mid=\sqrt{k_{1}^{2}+k_{2}^{2}+k_{3}^{2}}\right\} .
$$

Then for any sequence $u_{n}$ with $u_{(0,0,0)}=0$

$$
\sum_{k+m+n=0}\left|u_{k}\right|\left|u_{m}\right|\left|u_{n}\right| \mathcal{X}(k, m, n) \leq C\left(\sum_{n}|n|^{\beta}\left|u_{n}\right|^{2}\right)^{1 / 2}\left(\sum_{k}|k|^{\gamma}\left|u_{k}\right|^{2}\right)^{1 / 2}\left(\sum_{m}\left|u_{m}\right|^{2}\right)^{1 / 2}
$$

where $C=6 \sqrt{2 C_{0}}$.

Consider the polynomial $P(k,-n-k, n)$,

$$
\begin{aligned}
& P(k,-n-k, n)=k_{3}^{4}|-n-k|^{4}|n|^{4}\left(1+\alpha^{2}|-n-k|^{2}\right)^{4}\left(1+\alpha^{2}|n|^{2}\right)^{4} \\
& +(-n-k)^{4}|k|^{4}|n|^{4}\left(1+\alpha^{2}|k|^{2}\right)^{4}\left(1+\alpha^{2}|n|^{2}\right)^{4} \\
& +n_{3}^{4}|k|^{4}|-n-k|^{4}\left(1+\alpha^{2}|k|^{2}\right)^{4}\left(1+\alpha^{2}|-n-k|^{2}\right)^{4} \\
& -2 k_{3}^{2}\left(-n_{3}-k_{3}\right)^{2}|k|^{2}|-n-k|^{2}|n|^{4}\left(1+\alpha^{2}|k|^{2}\right)^{2} \\
& \left(1+\alpha^{2}|-n-k|^{2}\right)^{2}\left(1+\alpha^{2}|n|^{2}\right)^{4} \\
& -2\left(-n_{3}-k_{3}\right)^{2} n_{3}^{2}|k|^{4}|-n-k|^{2}|n|^{2}\left(1+\alpha^{2}|k|^{2}\right)^{4} \\
& \left(1+\alpha^{2}|-n-k|^{2}\right)^{2}\left(1+\alpha^{2}|n|^{2}\right)^{2} \\
& -2 k_{3}^{2} n_{3}^{2}|k|^{2}|-n-k|^{4}|n|^{2}\left(1+\alpha^{2}|k|^{2}\right)^{2}\left(1+\alpha^{2}|-n-k|^{2}\right)^{4} \\
& \left(1+\alpha^{2}|n|^{2}\right)^{2} \text {. }
\end{aligned}
$$

The highest power of $k_{3}$ is 24 , and $P(k,-n-k, n)$ is a polynomial of degree 24 in $k_{3}$. The coefficient at $k_{3}^{24}$ is $\alpha^{16} n_{3}^{4}$ which can be obtained from the term

$$
n_{3}^{4}|k|^{4}|-n-k|^{4}\left(1+\alpha^{2}|k|^{2}\right)^{4}\left(1+\alpha^{2}|-n-k|^{2}\right)^{4},
$$


which does not vanish because $k_{3} \neq 0, m_{3} \neq 0$ and $n_{3} \neq 0$. Thus, for fixed $k_{1}, k_{2}, n$, there are at most $24 k_{3}$ satisfying $P(k,-n-k, n)=0$. So, if we estimate the sum (4.17) with $\alpha=1$,

$$
\begin{aligned}
\sum_{2^{i} \leq|k|<2^{i+1}}\left(k_{1}^{2}+k_{2}^{2}+k_{3}^{2}\right)^{-\frac{1}{2}} \mathcal{X}(k,-k-n, n) & \leq 24+24 \sum_{0<\left|k_{h}\right|<2^{i+1}}\left(k_{1}^{2}+k_{2}^{2}\right)^{-\frac{1}{2}} \\
& =24+24 \sum_{0<\left|k_{h}\right|<2^{i+1}}\left|k_{h}\right|^{-1} \\
& \leq C_{0} 2^{i},
\end{aligned}
$$

where $C_{0}$ is an absolute constant independent from $\alpha$. Note that the only difference with BMN's [7] is the coefficient 24, which replaces the coefficient 8 in [7]. Thus, the inequality (4.18) holds with $\gamma=\beta=1$.

Let $v_{k}=|k|\left|w_{k}^{\perp}\right|$ and similarly for $m, n$ in the inequality (4.15). Then using Corollary 4.6 we obtain the following estimation, uniformly in $\alpha$ :

$$
\begin{aligned}
\left|<\tilde{B}_{I I I}^{\alpha}\left(w^{\perp}, w^{\perp}\right), A w^{\perp}>\right| & \leq c \sum_{k+m+n=0}\left|v_{k}\right|\left|v_{m}\right|\left|v_{n}\right| \mathcal{X}(k, m, n) \\
& \leq \tilde{c}\left(\sum_{n}|n|\left|v_{n}\right|^{2}\right)^{1 / 2}\left(\sum_{k}|k|\left|v_{k}\right|^{2}\right)^{1 / 2}\left(\sum_{m}\left|v_{m}\right|^{2}\right)^{1 / 2} \\
& =\tilde{c}\|v\|_{\frac{1}{2}}^{2}|v| \\
& \leq \tilde{c}\left\|w^{\perp}\right\|_{\frac{3}{2}}^{2}\left\|w^{\perp}\right\| \\
& \leq C\left\|w^{\perp}\right\|^{2}\left\|w^{\perp}\right\|_{2} .
\end{aligned}
$$

Here, we used $\|v\|_{\frac{1}{2}}=\left\|w^{\perp}\right\|_{\frac{3}{2}}$ and $|v|=\left\|w^{\perp}\right\|$. Also, for the last inequality, we applied the interpolation inequality, $\left\|w^{\perp}\right\|_{\frac{3}{2}}^{2} \leq c\left\|w^{\perp}\right\|\left\|w^{\perp}\right\|_{2}$. Therefore, we have proved the following:

Theorem 4.9. Let $w^{\perp} \in H^{2}$. Then

$$
\left|<\tilde{B}_{I I I}^{\alpha}\left(w^{\perp}, w^{\perp}\right), A w^{\perp}>\right| \leq C_{I I I}\left\|w^{\perp}\right\|_{2}\left\|w^{\perp}\right\|^{2} .
$$

The estimates are uniform in $\alpha$.

From the estimate (4.19) we easily obtain the existence of a unique regular solution of baroclinic equations, uniformly in $\alpha$. Estimate (4.19) is similar to that of 2D Navier-Stokes equations with Dirichlet boundary conditions. The procedure of proof is exactly the same as the one in the proof of Theorem 3.2 of [7].

Theorem 4.10. Let $w^{\perp}(0) \in H^{1}$ and let $F^{\perp}$ be a force with $\left|F^{\perp}\right|^{2} \leq M_{0 F}$. Then, for any $T \geq 0$, there exists a unique global regular solution $w^{\perp} \in L^{\infty}\left(0, T ; H^{1}\right) \cap$ $L^{2}\left(0, T ; H^{2}\right)$ of baroclinic limit equations (3.47) and (3.48), with the norm estimates uniform in $\alpha$.

\footnotetext{
5. Local existence of solutions of 3D rotating Navier-Stokes- $\alpha$ equations, independent of $\alpha$

In this section we prove the existence of a solution of the problem (1.1)-(1.2) on a small (but f-independent) time interval. We assess the estimates on nonlinear terms of RNS- $\alpha$ equations to get the same estimates as the one of RNSE in Ch4 of [6]. That makes it possible to follow the exact same procedure developed in Ch4 of [6]. Now
} 
we start off by recalling RNS- $\alpha$ equations in a Fourier space: for each wave number $n$, Eq. (1.1) takes the form

$$
\frac{\partial V_{n}}{\partial t}+\frac{1}{1+\alpha^{2}|\check{n}|^{2}} f\left(P_{L} J P_{L}\right)_{n} V_{n}+\nu|\check{n}|^{2} V_{n}+i P_{n} \sum_{k+m=n}\left(\left(U_{k} \cdot \check{m}\right) V_{m}+V_{k}^{(j)} \check{m} U_{m}^{(j)}\right)=F_{n} .
$$

Recall that the action of $A^{s}$ in a Fourier space is multiplication by $|\check{n}|^{2 s}$. Multiplying Eq. (5.1) by $V_{n}^{*}|\check{n}|^{2 s}\left(V_{n}^{*}=V_{-n}\right)$ and using the skew-symmetry of $\left(P_{L} J P_{L}\right)_{n}$ (Lemma $2.3)$, we obtain

$$
\begin{aligned}
\frac{1}{2} \frac{\partial}{\partial t} \sum_{n}\left|V_{n}\right|^{2}|\check{n}|^{2 s}+\nu\|V\|_{s+1}^{2}= & -i \sum_{k+m+n=0}\left(U_{k} \cdot \check{m}\right)\left(V_{m} \cdot V_{n}|\check{n}|^{2 s}\right) \\
& -i \sum_{k+m+n=0} V_{k}^{(j)} U_{m}^{(j)}\left(\check{m} \cdot V_{n}|\check{n}|^{2 s}\right) \\
& +\sum_{n} F_{n} \cdot V_{-n}|\check{n}|^{2 s} .
\end{aligned}
$$

Here we assume the summation is over $|\check{k}| \leq R,|\check{m}| \leq R$ and $|\check{n}| \leq R$ for Galerkin approximations and then will take $R \rightarrow \infty$. The following property holds:

LEMMA 5.1. $\left(U_{k} \cdot \check{m}\right) V_{m} \cdot V_{n}$ is skew-symmetric in $n, m$ :

$$
\sum_{k+m+n=0}\left(U_{k} \cdot \check{m}\right)\left(V_{m} \cdot V_{n}\right)|\check{m}|^{s}|\check{n}|^{s}=0 .
$$

Proof. Interchanging $n$ and $m$ in the summation and then using the divergence free condition $\check{k} \cdot U_{k}=0$, we obtain

$$
\begin{aligned}
\sum_{k+m+n=0}\left(U_{k} \cdot \check{m}\right)\left(V_{m} \cdot V_{n}\right)|\check{m}|^{s}|\check{n}|^{s} & =\sum_{k+m+n=0}\left(U_{k} \cdot \check{n}\right)\left(V_{n} \cdot V_{m}\right)|\check{n}|^{s}|\check{m}|^{s} \\
& =-\sum_{k+m+n=0}\left(U_{k} \cdot(\check{k}+\check{m})\right)\left(V_{n} \cdot V_{m}\right)|\check{n}|^{s}|\check{m}|^{s} \\
& =-\sum_{k+m+n=0}\left(U_{k} \cdot \check{m}\right)\left(V_{m} \cdot V_{n}\right)|\check{m}|^{s}|\check{n}|^{s} .
\end{aligned}
$$

Therefore, $\sum_{k+m+n=0}\left(U_{k} \cdot \check{m}\right)\left(V_{m} \cdot V_{n}\right)|\check{m}|^{s}|\check{n}|^{s}=0$.

The second term in Eq. (5.2) doesn't have the skew-symmetry in $n$ and $m$, and we have to get the uniform estimate in $\alpha$ of it. We introduce the space of periodic functions $H_{p}^{s}$ (when $p=2$ it coincides with the Sobolev space $H^{s}=H_{2}^{s}$ ) with the norm defined on Fourier coefficients $u_{k}$ as follows:

$$
\|u\|_{H_{p}^{s}}^{p}=\sum_{k \in \mathbb{Z}^{3}}|\hat{k}|^{p s}\left|u_{k}\right|^{p}
$$

Note that $H_{p}^{s} \subset H_{q}^{s}$ if $p \leq q$ because the wave numbers lattice is discrete. Then,

LEMMA 5.2.

$$
\left.\left|\sum_{k+m+n=0} V_{k}^{(j)} U_{m}^{(j)}\left(\check{m} \cdot V_{n}\right)\right| \check{m}\right|^{s}|\check{n}|^{s} \mid \leq C_{2}(\beta)\|V\|_{\beta}\|V\|_{s}^{2}
$$


where $C_{2}(\beta)=\left(\sum_{k}|\breve{k}|^{2-2 \beta}\right)^{1 / 2} \leq\left(\sum_{k}|k|^{2-2 \beta}\right)^{1 / 2}<\infty$ if $\beta>\frac{5}{2}$

Proof. We use $\check{n} \cdot V_{n}=(\check{k}+\check{m}) \cdot V_{n}=0$ to get

$$
\begin{aligned}
\left.\left|\sum_{k+m+n=0} V_{k}^{(j)} U_{m}^{(j)}\left(\check{m} \cdot V_{n}\right)\right| \check{m}\right|^{s}|\check{n}|^{s} \mid & =\left.\left|\sum_{k+m+n=0} V_{k}^{(j)} U_{m}^{(j)}\left((-\check{k}) \cdot V_{n}\right)\right| \check{m}\right|^{s}|\check{n}|^{s} \mid \\
& \leq \sum_{k+m+n=0}|\check{k}|\left|V_{k}^{(j)}\right||\check{m}|^{s}\left|U_{m}^{(j)}\right||\check{n}|^{s}\left|V_{n}\right| \\
& \leq \sum_{k+m+n=0}|\check{k}|\left|V_{k}^{(j)}\right||\check{m}|^{s}\left|V_{m}^{(j)}\right||\check{n}|^{s}\left|V_{n}\right| \\
& \leq\|V\|_{H_{1}^{1}}\|V\|_{s}^{2} \\
& \leq C_{2}(\beta)\|V\|_{\beta}\|V\|_{s}^{2} .
\end{aligned}
$$

Now, we formulate the estimate on a nonlinear term.

LEMMA 5.3.

$$
\left|<B_{\alpha}(V, V), A^{s} V>\right| \leq C(s)\|V\|_{\beta}\|V\|_{s}^{2}
$$

with $s \geq \beta>\frac{5}{2}$ and $\beta$ is independent of $s$. This estimate is independent from $\alpha$.

Proof. The estimation relies on the inequality ||$\check{k}+\left.\check{m}\right|^{s}-|\check{m}|^{s} \mid \leq C_{1}(s)\left(|\check{m}|^{s-1}|\check{k}|+\right.$ $\left.|\check{k}|^{s}\right)$ and the identity $|\check{k}+\check{m}|^{s}=|\check{m}|^{s}+\left(|\check{k}+\check{m}|^{s}-|\check{m}|^{s}\right)$.

(i)

$$
\begin{aligned}
\sum_{k+m+n=0}\left(U_{k} \cdot \check{m}\right)\left(V_{m} \cdot V_{n}|\check{n}|^{2 s}\right)=\sum_{k+m+n=0}\left(U_{k} \cdot \check{m}\right)\left(V_{m} \cdot V_{n}\right)|\check{k}+\check{m}|^{s}|\check{n}|^{s} \\
=\sum_{k+m+n=0}\left(U_{k} \cdot \check{m}\right)\left(V_{m} \cdot V_{n}\right)\left[|\check{m}|^{s}+\left(|\check{k}+\check{m}|^{s}-|\check{m}|^{s}\right)\right]|\check{n}|^{s} \\
=\sum_{k+m+n=0}\left(U_{k} \cdot \check{m}\right)\left(V_{m} \cdot V_{n}\right)|\check{m}|^{s}|\check{n}|^{s} \\
\quad+\sum_{k+m+n=0}\left(U_{k} \cdot \check{m}\right)\left(V_{m} \cdot V_{n}\right)\left(|\check{k}+\check{m}|^{s}-|\check{m}|^{s}\right)|\check{n}|^{s} \\
=\sum_{k+m+n=0}\left(U_{k} \cdot \check{m}\right)\left(V_{m} \cdot V_{n}\right)\left(|\check{k}+\check{m}|^{s}-|\check{m}|^{s}\right)|\check{n}|^{s}
\end{aligned}
$$

We used Lemma 5.1 for the last equality. The right hand side of the above equation can be estimated as:

$$
\begin{aligned}
& \left.\left|\sum_{k+m+n=0}\left(U_{k} \cdot \check{m}\right)\left(V_{m} \cdot V_{n}\right)\left(|\check{k}+\check{m}|^{s}-|\check{m}|^{s}\right)\right| \check{n}\right|^{s} \mid \\
& \leq C_{1}(s) \sum_{k+m+n=0}|\breve{k}|\left|U_{k}\right||\check{m}|^{s}\left|V_{m}\right||\check{n}|^{s}\left|V_{n}\right| \\
& +C_{1}(s) \sum_{k+m+n=0}|\check{k}|^{s}\left|U_{k}\right||\check{m}|\left|V_{m}\right||\check{n}|^{s}\left|V_{n}\right| \\
& \leq C_{1}(s) \sum_{k+m+n=0}|\check{k}|\left|V_{k}\right||\check{m}|^{s}\left|V_{m}\right||\check{n}|^{s}\left|V_{n}\right| \\
& +C_{1}(s) \sum_{k+m+n=0}|\check{k}|^{s}\left|V_{k}\right||\check{m}|\left|V_{m}\right||\check{n}|^{s}\left|V_{n}\right| .
\end{aligned}
$$


By Young's inequality for convolutions $\|f * g\|_{L^{r}} \leq\|f\|_{L^{p}}\|g\|_{L^{q}}$, where $\frac{1}{p}+$ $\frac{1}{q}=1+\frac{1}{r}$, we get

$$
\begin{aligned}
\sum_{k+m+n=0}|\check{k}|\left|V_{k}\right||\check{m}|^{s}\left|V_{m}\right||\check{n}|^{s}\left|V_{n}\right| & \leq\left[\sum_{n}\left\{\sum_{k+m=-n}|\check{k}|\left|V_{k}\right||\check{m}|^{s}\left|V_{m}\right|\right\}^{2}\right]^{\frac{1}{2}}\|V\|_{s} \\
& \leq\|V\|_{H_{1}^{1}}\|V\|_{s}^{2} \\
& \leq C_{2}(\beta)\|V\|_{\beta}\|V\|_{s}^{2}
\end{aligned}
$$

where $C_{2}(\beta)=\left(\sum_{k}|\breve{k}|^{2-2 \beta}\right)^{1 / 2} \leq\left(\sum_{k}|k|^{2-2 \beta}\right)^{1 / 2}<\infty$ if $\beta>\frac{5}{2}$ (for the first inequality we used $\left.a_{1}, a_{2}, a_{3} \leq 1\right)$. The other sum has the same estimate. Thus

$$
\left.\left|\sum_{k+m+n=0}\left(U_{k} \cdot \check{m}\right)\left(V_{m} \cdot V_{n}\right)\left(|\check{k}+\check{m}|^{s}-|\check{m}|^{s}\right)\right| \check{n}\right|^{s} \mid \leq 2 C_{1}(s) C_{2}(\beta)\|V\|_{\beta}\|V\|_{s}^{2} .
$$

(ii) Note that

$$
\begin{aligned}
\sum_{k+m+n=0} V_{k}^{(j)} U_{m}^{(j)}\left(\check{m} \cdot V_{n}|\check{n}|^{2 s}\right) & \\
= & \sum_{k+m+n=0} V_{k}^{(j)} U_{m}^{(j)}\left(\check{m} \cdot V_{n}\right)\left[|\check{k}+\check{m}|^{s}-|\check{m}|^{s}\right]|\check{n}|^{s} \\
& +\sum_{k+m+n=0} V_{k}^{(j)} U_{m}^{(j)}\left(\check{m} \cdot V_{n}\right)|\check{m}|^{s}|\check{n}|^{s}
\end{aligned}
$$

Then

$$
\begin{aligned}
\mid \sum_{k+m+n=0} & V_{k}^{(j)} U_{m}^{(j)}\left(\check{m} \cdot V_{n}\right)\left[|\check{k}+\check{m}|^{s}-|\check{m}|^{s}\right]|\check{n}|^{s} \mid \\
& \leq C_{1}(s)\left(|\check{m}|^{s-1}|\check{k}|+|\check{k}|^{s}\right)\left|V_{k}^{(j)}\right|\left|V_{m}^{(j)}\right||\check{m}|\left|V_{n}\right||\check{n}|^{s} \\
& =C_{1}(s)|\check{m}|^{s}\left|V_{m}^{(j)}\right||\check{k}|\left|V_{k}^{(j)}\right||\check{n}|^{s}\left|V_{n}\right| \\
& \quad+C_{1}(s)|\check{k}|^{s}\left|V_{k}^{(j)}\right||\check{m}|\left|V_{m}^{(j)}\right||\check{n}|^{s}\left|V_{n}\right| \\
& \leq 2 C_{1}(s)\|V\|_{H_{1}^{1}}\|V\|_{s}^{2} \\
\leq & 2 C_{1}(s) C_{2}(\beta)\|V\|_{\beta}\|V\|_{s}^{2} .
\end{aligned}
$$

Thus, the inequality (5.3) and Lemma 5.2 give the estimate

$$
\left|\sum_{k+m+n=0} V_{k}^{(j)} U_{m}^{(j)}\left(\check{m} \cdot V_{n}|\check{n}|^{2 s}\right)\right| \leq \tilde{C}(s)\|V\|_{\beta}\|V\|_{s}^{2} .
$$

Combined (i) and (ii) complete the proof.

Now we establish the local existence of solutions of the equations.

Theorem 5.4. Let $0 \leq \nu \leq 1, s>5 / 2, V(0) \in H^{s}$. Assume that $F \in H^{s}$ and that

$$
\|V(0)\|_{s} \leq M_{0 s} ; \quad\|F\|_{s} \leq M_{0 s F} .
$$

Then there exists $T_{s}>0$ such that there exists a unique local solution of Eq. (1.1)-(1.3) which is bounded in $L^{\infty}\left(0, T_{s} ; H^{s}\right)$ and satisfies

$$
\|V(t)\|_{s}^{2} \leq M_{s}^{2}, 0 \leq t \leq T_{s} ; \quad \nu \int_{0}^{T_{s}}\|V(t)\|_{s+1}^{2} d t \leq M_{s}^{2}
$$


where $M_{s}, T_{s}$ do not depend on $\nu, \Omega, a_{2}, a_{3}$ but depend on $M_{0 s}, M_{0 s F}$. Here we assume that $M_{s} \geq M_{0 s} \geq M_{0 s F} \geq 1, T_{s} \leq 1$. These estimates are uniform in $\alpha$.

Proof. Existence is proved using Galerkin approximations. The approximations are equivalent to considering Eq. (5.1) with $|\check{k}| \leq R,|\check{m}| \leq R$. Later we take $R \rightarrow \infty$. obtain

By multiplying (5.1) by $V_{n}^{*}|\check{n}|^{2 s}$ we could get (5.2). Together with Lemma 5.3 we

$$
\frac{\partial}{\partial t}\|V\|_{s}^{2}+2 \nu\|V\|_{s+1}^{2} \leq C(s, \beta)\|V\|_{\beta}\|V\|_{s}^{2}+\|F\|_{s}^{2}+\|V\|_{s}^{2}
$$

where a constant $C(s, \beta)=\max \left\{4 C_{1}(s) C_{2}(\beta), 4 C_{2}(\beta)\right\}$. By Gronwall's inequality

$$
\|V(t)\|_{s}^{2} \leq\left[\|V(0)\|_{s}^{2}+M_{0 s F}^{2}\right] \exp \left(\int_{0}^{T_{s}}\left[C(s, \beta)\|V(s)\|_{\beta}+1\right] d s\right),
$$

where $s \geq \beta>5 / 2$. Note that $\beta$ does not depend on $s$ so that $\beta$ can be fixed independently of $s$. We put $\beta=s$. Then Eq. (5.5) becomes

$$
\frac{\partial}{\partial t}\|V\|_{s}^{2}+2 \nu\|V\|_{s+1}^{2} \leq C(s)\|V\|_{s}^{3}+M_{0 s F}^{2}+\|V\|_{s}^{2} .
$$

Let $y=\|V\|_{s}^{2}+M_{0 s}^{2}$ and then $y \geq 1$. The inequality (5.6) provides

$$
\frac{d y}{d t}+2 \nu\|V\|_{s+1}^{2} \leq[C(s)+1] y^{3 / 2}
$$

Solving the ODE gives the inequality

$$
y(t) \leq\left[\frac{1}{2 M_{0 s}^{2}}-(C(s)+1) t\right]^{-2}
$$

on the interval $\left[0, t^{*}\right]$, where $t^{*}=1 /\left[4 M_{0 s}(C(s)+1)\right]$. Taking $T_{s}=t^{*}$ yields

$$
\|V\|_{s}^{2} \leq 16 M_{0 s}^{2}, \quad 0 \leq\left[4 M_{0 s}(C(s)+1)\right]^{-1} .
$$

Integrating (5.7) we obtain on the same interval

$$
2 \nu \int_{0}^{T_{s}}\|V\|_{s+1}^{2} d t \leq 66(C(s)+1)\left[M_{0 s}\right]^{3}, \quad T_{s} \leq\left[4 M_{0 s}(C(s)+1)\right]^{-1} .
$$

The inequalities (5.7) and (5.8) imply (5.4) with the appropriate choice of $M_{s}, T_{s}$.

Using these estimates, we can prove the existence of a solution of the problem in a standard way with $R \rightarrow \infty$. The solution satisfies the same estimates and the theorem is proved.

REMARK 5.5. Theorem 5.4 is true for the usual rotating Navier-Stokes equations (that is, when $\alpha=0$ ). See Ch4 in [6] for details.

\section{Global regularity of $3 \mathrm{D}$ rotating Navier-Stokes- $\alpha$ equations for large} $f$ and finite $\alpha$

In this section, we establish the existence and global regularity of solutions of 3D rotating Navier-Stokes- $\alpha$ equations (1.1)-(1.3) for $f$ large enough which is uniform in $\alpha$. Our regularity estimates are uniform in $\alpha$, which is at the very heart of our results and make them distinctive from the previous literature. Typically, only weak 
convergence of the solution of the $3 D$ NS- $\alpha$ equations to some weak Leray solution had been obtained in the limit $\alpha \rightarrow 0$ ([22]). We use techniques developed by A. Babin, A. Mahalov and B. Nicolaenko in [6] - [12] to establish the existence and global regularity for the real velocity field $V$, which exist uniformly in $\alpha$. The uniformity in $\alpha$ make it possible to establish the existence of absorbing balls, uniformly in $\alpha$, and the strong convergence of solutions of 3D rotating Navier-Stokes- $\alpha$ equations with large fixed rotation $f=2 \Omega$ as $\alpha$ vanishes.

The proof of global regularity relies on the global regularity of the limit $\alpha$ equations and techniques for convergence theorems as $f \rightarrow \infty$. From now on we assume $0 \leq \alpha<\alpha_{M}$, where $\alpha_{M}$ is some maximum of $\alpha$ since we are interested in the limit $\alpha \rightarrow 0^{+}$. Let $V_{s}(0) \in H^{\sigma}$ be smooth initial data and let $F_{s}$ be a smooth force such that $F_{s}(t) \in H^{\sigma}, \sigma>\beta+4, \beta>5 / 2$, which are close to $V(0)$ and $F$. We approximate initial data in $H^{\beta}$ by smoother initial data in $H^{\sigma}$ :

$$
\left\|V(0)-V_{s}(0)\right\|_{\beta} \leq \epsilon
$$

Further, we assume that $F$ is approximated by $F_{s}$. We denote $F^{\prime}=F-F_{s}$ and assume

$$
\left\|F^{\prime}\right\|_{\beta-1}^{2} \leq \epsilon^{2}
$$

with $\beta>5 / 2$.

Using the previous results on equations we will find a solution $V_{s}(t)$ of (1.1)-(1.3) in $H^{\sigma}, \sigma>\beta+4, \beta>5 / 2$ with mollified data which satisfies $\epsilon$-independent estimates in $H^{\beta}$ for large $f$ and finite $\alpha$. The solutions $V_{s}, V$ satisfy equations of the form

$$
\begin{gathered}
\partial_{t} V(t)=B_{\alpha}(V, V)-\nu A V-f P_{L} J P_{L} \mathcal{R}_{\alpha} V+F \\
\partial_{t} V_{s}(t)=B_{\alpha}\left(V_{s}, V_{s}\right)-\nu A V_{s}-f P_{L} J P_{L} \mathcal{R}_{\alpha} V_{s}+F
\end{gathered}
$$

with the same Stokes operator $A$ and bilinear operator $B_{\alpha}$ and different (but close) initial data. The difference $\Xi(t)=V(t)-V_{s}(t)$ satisfies the equation

$$
\begin{gathered}
\partial_{t} \Xi(t)=B_{\alpha}\left(\Xi, V_{s}\right)+B_{\alpha}\left(V_{s}, \Xi\right)+B_{\alpha}(\Xi, \Xi)-\nu A \Xi-f P_{L} J P_{L} \Xi+F^{\prime}, \\
\Xi(0)=V(0)-V_{s}(0)
\end{gathered}
$$

with a small forcing term $F^{\prime}=F-F_{s}$ and small initial data $\Xi(0)$.

We start out by generalizing Lemma 5.1 in [7]. Once it is constructed, theorems in Ch5 of [7] can be adapted, and hence the global existence and regularity follow. Recall that, for each fixed wave number $n$,

$$
B_{\alpha}^{o s c}(f t, v, v)_{n}=\sum_{\substack{l, k+m=n \\ D_{l}(k, m, n) \neq 0}} e^{i f t D_{l}(k, m, n)} Q_{k m n l}\left(v_{k}, v_{m}\right)
$$

where $l=1, \ldots, 8$, and $Q_{k m n l}\left(v_{k}, v_{m}\right)$ is a bilinear form in $v_{k}, v_{m} \in \mathbb{C}^{3} . \quad B_{\alpha}^{o s c}(f t, v, v)$ contains all nonresonant terms ( $f t$-dependent terms). Here, the bilinear function $Q_{k m n l}\left(v_{k}, v_{m}\right)$ is expressed by

$$
Q_{k m n l}\left(v_{k}, v_{m}\right)=i P_{n} \sum_{k+m=n}\left[\left(\left(\mathcal{R}_{\alpha} v\right)_{k} \cdot \check{m}\right) v_{m}+v_{k}^{(j)} \check{m}\left(\mathcal{R}_{\alpha} v\right)_{k}^{(j)}\right]
$$


Then

$$
\begin{aligned}
\left|\left(\mathcal{R}_{\alpha} v\right)_{k} \cdot \check{m} v_{m}\right| & \leq\left|\left(\mathcal{R}_{\alpha} v\right)_{k}\right||\check{m}|\left|v_{m}\right| \\
& \leq \frac{1}{1+\alpha^{2}|\check{k}|^{2}}\left|v_{k}\right||\check{m}|\left|v_{m}\right| \\
& \leq|\check{m}|\left|v_{k}\right|\left|v_{m}\right| \\
\left|v_{k}^{(j)} \check{m}\left(\mathcal{R}_{\alpha} v\right)_{k}^{(j)}\right| & \leq\left|v_{k}^{(j)}\right||\check{m}|\left|\left(\mathcal{R}_{\alpha} v\right)_{m}^{(j)}\right| \\
& \leq\left|v_{k}^{(j)}\right||\check{m}| \frac{1}{1+\alpha^{2}|\check{m}|^{2}}\left|v_{m}^{(j)}\right| \\
& \leq|\check{m}|\left|v_{k}\right|\left|v_{m}\right| .
\end{aligned}
$$

Since the summation has finitely many terms for each fixed $n$, the bilinear function has the following inequality,

$$
\left|Q_{k m n l}\left(v_{k}, v_{m}\right)\right| \leq C_{Q}|\check{m}|\left|v_{k}\right|\left|v_{m}\right| .
$$

This estimate satisfies the condition of Lemma 5.1 in [7], and the corresponding lemma follows uniformly in $\alpha$ :

LEMma 6.1. Let $\mathbb{X} \subset\left(\mathbb{Z}^{9}\{0\}\right) \times\{1, \ldots, 8\}=\left(\left(\mathbb{Z}_{k}^{3} \times \mathbb{Z}_{m}^{3} \times \mathbb{Z}_{n}^{3}\right)\{0\}\right) \times\{1, \ldots, 8\} . \quad$ Let $Q_{k m n l}$ be a bilinear function from $\mathbb{C}^{3} \times \mathbb{C}^{3}$ to $\mathbb{C}^{3}$ which depends on $(k, m, n) \in \mathbb{Z}^{9}$ and on $l=1, \ldots, 8$ and satisfies for $n=k+m$ :

$$
\left|Q_{k m n l}\left(u_{k}, v_{m}\right)\right| \leq C_{Q}|\check{m}|\left|u_{k}\right|\left|v_{m}\right| .
$$

Let

$$
\left(B_{Q}(u, v)\right)_{n}=\sum_{\substack{k+m=n \\(k, m, n, l) \in \mathbb{X}}} Q_{k m n l}\left(u_{k}, v_{m}\right) .
$$

Let $\beta \geq 0$. Then with $C_{8}=C_{8}(\beta)$ we have

$$
\begin{aligned}
\left|<B_{Q}(u, v), A^{\beta} w>\right| & \leq C_{Q} C_{8}\|w\|_{\beta+1}\left[\|u\|_{1 / 2}\|v\|_{\beta+1}+\|u\|_{\beta+1 / 2}\|v\|\right] \\
& \leq \nu\|w\|_{\beta+1}^{2} / 8+C\left[\|u\|_{\sigma}^{2}\|v\|_{\sigma}^{2}\right] / \nu
\end{aligned}
$$

for arbitrary $\nu>0$ when $\sigma \geq \max \{\beta+1,1\}$. Moreover, if $0 \leq \beta<3 / 2$

$$
\begin{aligned}
\left|<B_{Q}(u, v), A^{\beta} w>\right| & \leq C_{Q} C_{8}\|w\|_{\beta+1}\|u\|_{3 / 4+\beta / 2}\|v\|_{3 / 4+\beta / 2} \\
& \leq \nu\|w\|_{\beta+1}^{2} / 8+C(1 / \nu)\|u\|_{3 / 4+\beta / 2}^{2}\|v\|_{3 / 4+\beta / 2}^{2} .
\end{aligned}
$$

If $0 \leq \beta<3 / 2$ and $\epsilon<<1$, we have

$$
\begin{aligned}
\left|<B_{Q}(v, w), A^{\beta} w>\right| & \leq C_{Q} C_{7}\|w\|_{\beta+1}\|w\|_{\beta+1-\epsilon}\|v\|_{1 / 2+\epsilon} \\
& \leq \nu\|w\|_{\beta+1}^{2} / 8+C \nu^{1-2 / \epsilon}\|v\|_{1 / 2+\epsilon}^{2 / \epsilon}\|w\|_{\beta}^{2} .
\end{aligned}
$$

All these estimates are uniform in $\alpha$.

Now we recapture theorems from Ch5 of [7]. The proofs can be carried over from Theorem 5.1 and Theorem 5.2 in [7].

First, the following theorem establishes the existence of regular solutions on large time intervals of 3D Navier-Stokes- $\alpha$ type systems with very small initial data and forcing, uniformly in $\alpha$ with the help of Lemma 6.1. 
TheOREm 6.2. Let $\beta>5 / 2, \nu>0, T_{0}>0$. Let the following hold:

$$
\left\|V(0)-V_{s}(0)\right\|_{\beta} \leq \epsilon
$$

and

$$
\left\|F^{\prime}\right\|_{\beta-1}^{2} \leq \epsilon^{2}
$$

Assume that

$$
\left\|V_{s}(t)\right\|_{\beta} \leq M_{s \beta}, 0 \leq t \leq T_{0}, \nu \int_{0}^{T_{0}}\left\|V_{s}(t)\right\|_{\beta+1}^{2} \leq M_{s \beta}^{2} ;
$$

and $\epsilon \leq \epsilon_{0}$, where $\epsilon_{0}$ depends on $M_{s \beta}, \beta, \nu, T_{0}$. Then a regular solution $\Xi$ of (6.4) exists in $H^{\beta}$ and

$$
\|\Xi(t)\|_{\beta} \leq C_{0} \epsilon, \nu \int_{0}^{T_{0}}\|\Xi(t)\|_{\beta+1}^{2} \leq C_{0}^{2} \epsilon^{2}, 0 \leq t \leq T_{0},
$$

where $C_{0}$ depends on $M_{s \beta}, \beta, \nu$. In particular, the estimates are uniform in $\alpha$.

The proof of the theorem is exactly as the one of Theorem 5.1 in [7], albeit with the uniform in $\alpha$ Lemma 6.1 .

Now we derive the existence theorem for Eq. (6.3) with smooth initial data in $H^{\sigma}, \sigma>\beta+4$, which includes a 3 -wave resonance operator.

THEOREM 6.3. Let $a_{1}, a_{2}, a_{3}$ be arbitrary and fixed; let $\beta>5 / 2, \nu>0, \sigma>\beta+4$ and let $V_{s}(t)$ be a solution of Eq. (1.1)-(1.3) with smooth initial data and forcing term such that

$$
\begin{array}{r}
\left\|V_{s}(0)\right\|_{\beta} \leq M_{\beta}, \quad\left\|F_{s}\right\|_{\beta-1}^{2} \leq\left(M_{\beta F}\right)^{2} ; \\
\left\|V_{s}(0)\right\|_{\sigma} \leq M_{\sigma F}, \quad\left\|F_{s}\right\|_{\sigma-1}^{2} \leq\left(M_{\sigma F}\right)^{2} ;
\end{array}
$$

Then, for every $f \geq f_{0}\left(M_{\beta}, M_{\beta F}, M_{\sigma F}, \nu, a_{1}, a_{2}, a_{3}\right)$, there exists a unique solution $V_{s}(t)$ for $0 \leq t<\infty$ such that

$$
\begin{array}{r}
\left\|V_{s}(t)\right\|_{\beta} \leq M_{\beta}^{\prime}, \quad 0 \leq t<\infty, \\
\nu \int_{0}^{T_{0}}\left\|V_{s}(t)\right\|_{\beta+1}^{2} d t \leq\left(M_{\beta}^{\prime}\right)^{2}
\end{array}
$$

where $M_{\beta}^{\prime}$ depends only on $M_{\beta}, M_{\beta F}, \nu, a_{1}, a_{2}, a_{3}$ but not on $M_{\sigma F}$. Moreover, these estimates are uniform in $\alpha$.

For the proof of Theorem 6.3 we need the lemma below, which is uniform in $\alpha$ thanks to the local existence Theorem 5.4 and the uniform in $\alpha$ Theorems 4.1, 4.3 , and 4.10 for the limit resonant $\alpha$-equations. Consider (Poincaré variable) $v_{s}(t)=$ $\exp \left(f P J P \mathcal{R}_{\alpha} t\right) V_{s}(t)$, where $V_{s}(t)$ is a solution of (6.3) with smooth initial data $V_{s}(0)$ and smooth force $F_{s}(t)$; consider $w(t)=\bar{w}(t)+w^{\perp}(t)$ solution of the limit equations (3.45) for $\bar{w}$ and (3.47) for $w^{\perp}$, with:

$$
\bar{w}(0)=\bar{V}_{s}(0), w^{\perp}(0)=V_{s}^{\perp}(0) .
$$

Let $r(t)=v_{s}(t)-w(t)$. Then we have: 
LEMma 6.4. Let $T_{\sigma}\left(M_{\sigma F}, \nu, a_{1}, a_{2}, a_{3}\right)$ be the local (small) time existence for a solution $V_{s}(t)$ of (6.3). Under conditions $\beta>5 / 2, \sigma-\beta>4$ we have

$$
\begin{array}{r}
\|r(t)\|_{\beta} \leq \delta(f), \quad \forall t \in\left[0, T_{\sigma}\right], \\
\nu \int_{0}^{T}\|r(t)\|_{\beta+1}^{2} d t \leq(\delta(f))^{2}, \quad \forall T \in\left[0, T_{\sigma}\right],
\end{array}
$$

where $\delta(f) \rightarrow 0$ as $f \rightarrow \infty ; T_{\sigma}$ does not depend on $f ; \delta(f)$ depends on $M_{\sigma F}, T_{\sigma}, \nu, \beta, \sigma$, $a_{1}, a_{2}, a_{3}$, but uniformly in $\alpha$.

We then obtain the proof of Theorem 6.3, exactly as in [7] but extending the existence of $V_{s}(t)$ on $0 \leq t<\infty$. For this extension, the "uniformly in $\alpha$ " of Theorem 5.4 (local existence) and above all Theorems 4.1, 4.3, and 4.10 (regularity of limit equations for $0 \leq t<\infty)$ is crucial.

We now proceed with the existence and regularity theorem for less smooth $V(0)$ and $F$.

THEOREM 6.5. Let the domain parameters $a=\left(a_{1}, a_{2}, a_{3}\right)$ be fixed but arbitrary, and let $\nu>0,0 \leq \alpha<\alpha_{M}, \beta>5 / 2$. Let $\|F\|_{\beta-1}^{2} \leq M_{\beta F}^{2},\|V(0)\|_{\beta} \leq \tilde{M}_{\beta}$, and $f$ large: $f \geq$ $f_{1}\left(\tilde{M}_{\beta}, M_{\beta F}, \nu, a_{1}, a_{2}, a_{3}\right)$. Then, for each such $f$, the solutions of Eq. (1.1)-(1.3) are regular for all $t$ such that $0 \leq t \leq T$ and $T$ arbitrary large, and

$$
\|V(t)\|_{\beta} \leq \tilde{M}_{\beta}^{\prime} \quad \forall t \in[0, T]
$$

where $\tilde{M}_{\beta}^{\prime}=\tilde{M}_{\beta}^{\prime}\left(\tilde{M}_{\beta}, M_{\beta F}, \nu, a_{1}, a_{2}, a_{3}, T\right)$. Moreover, the estimates are uniform in $\alpha$.

Proof. We sketch the proof following the line of Theorem 5.3 in [7] albeit for $\beta>5 / 2$ instead of $1 / 2$ and without using the $L^{2}$ absorbing ball and $t^{*}$ entry time in $H^{1}$. The theorem is restricted to $\beta>5 / 2$ since we do not have any uniform in $\alpha$ estimates for $V$ in $L^{2}$, nor in $H^{1}$ ( and a fortiori in $H^{\beta}, 0<\beta<5 / 2$.) The root of the problem lies in the lack of classical energy conservation of $V$ for the inviscid, unforced $3 \mathrm{D}$ Euler- $\alpha$ equations. Only, $H^{-1}$ norm is conserved in $H^{1}$ (see p5-6 in Section 2). Otherwise, the theorem is proved with the help of the uniform in $\alpha$ Lemma 6.1, Theorem 6.2, Lemma 6.4 and Theorem 6.3.

We approximate the force $F$ and initial data $V(0)$ by smooth functions $F_{s}$ and $V_{s}(0)$ in $H^{\sigma}, \sigma>\beta+4$ so that:

$$
\|\Xi(0)\|_{\beta}=\left\|V(0)-V_{s}(0)\right\|_{\beta} \leq \eta,\left\|V_{s}(0)\right\|_{\sigma} \leq M_{\eta} .
$$

Moreover, the inequality (6.1) holds and, with $F^{\prime}=F-F_{s}$ :

$$
\left\|F^{\prime}(t)\right\|_{\sigma} \leq M_{\eta}
$$

where $M_{\eta}$ depends on $\tilde{M}_{\beta}, M_{\beta F}, \eta$ and $T$ only. We then consider the RNS- $\alpha$ equations with smooth initial data $V_{s}(0)$ and force $F_{s}$ in $H^{\sigma}$, which satisfy (6.8) and (6.9) in Theorem 6.3 with $M_{\beta} \leq \tilde{M}_{\beta}+M_{\eta},\left\|V_{s}(0)\right\|_{\sigma} \leq M_{\eta}$, and $M_{\sigma F} \leq M_{\beta F}+M_{\eta}$. We then apply Theorem 6.3 on $[0, T]$. The $H^{\sigma}$ norms of these smooth functions are bounded by ( a possibly large) constant $M_{\eta}$ depending on this fixed $\eta$ and $\tilde{M}_{\beta}, M_{\beta F}$. After that, we choose $f>f_{1}\left(\tilde{M}_{\beta}, M_{\beta F}, \eta, \nu, a_{1}, a_{2}, a_{3}\right)$ such that we have (6.10) and (6.10) in Theorem 6.3 for solutions $V_{s}(t)$ on $[0, T]$ of equations with smooth data. We then apply Theorem 6.2 with $\epsilon$ replaced by $\eta, M_{s \beta}$ by $\tilde{M}_{\beta}+\eta$ and $T_{0}$ by $T$; we obtain a regular solution $\Xi$ of (6.4) such that

$$
\|\Xi(t)\|_{\beta} \leq C_{0} \eta, \nu \int_{0}^{T}\|\Xi(\tau)\|_{\beta+1}^{2} d \tau \leq C_{0}^{2} \eta^{2}, 0 \leq t \leq T,
$$


where $C_{0}$ depends on $\tilde{M}_{\beta}, \beta$ and $\nu$. We now choose $\eta$ so small that we have $C_{0} \eta \leq \epsilon$ in Theorem 6.2, and we obtain

$$
\|V(t)\|_{\beta} \leq\left\|V_{s}(t)\right\|_{\beta}+\|\Xi(t)\|_{\beta} \leq M_{\beta}^{\prime}+\epsilon,
$$

where $M_{\beta}^{\prime}$ depends only on $\tilde{M}_{\beta}, M_{\beta F}, \nu, a_{1}, a_{2}, a_{3}, T$, but not on $M_{\eta}$. Finally, we obtain Theorem 6.5 with $\tilde{M}_{\beta}^{\prime}=M_{\beta}^{\prime}+\epsilon$.

Corollary 6.6. Let the conditions of Theorem 6.5 be satisfied. Let $\tilde{B}_{\beta}$ be a ball in $H^{\beta}, \beta>5 / 2$ with radius of $\tilde{B}_{\beta} \leq \tilde{M}_{\beta}$. Then, for $T$ given arbitrary large, we have

$$
\left\|S(t) \tilde{B}_{\beta}\right\|_{\beta} \leq \tilde{M}_{\beta}^{\prime}, \forall t \in[0, T]
$$

where $\tilde{M}_{\beta}^{\prime}=\tilde{M}_{\beta}^{\prime}\left(\tilde{M}_{\beta}, M_{\beta F}, \nu, a_{1}, a_{2}, a_{3}, T\right)$. Moreover, the estimates are uniform in $\alpha$.

Proof. As $\beta>5 / 2$, we can easily show that the semiflow $S(t)$ is compact on $H^{\beta}$ and take $\tilde{B}_{\beta}$ compact in $H^{\beta}$, modulo a small translation in time (smoothing property of the 3 D NSE, cf. [6] , [18]). We then cover $\tilde{B}_{\beta}$ with a finite set $\left\{V_{i}(0)\right\}, i=1,2, \ldots, N(\eta)$, such that, $\forall V(0) \in \tilde{B}_{\beta}$,

$$
\left\|V(0)-V_{i}(0)\right\|_{\beta} \leq \eta / 2 .
$$

For each $V_{i}(0)$, we apply Theorem 6.5 with $\left\|V_{s, i}(0)-V_{i}(0)\right\|_{\beta} \leq \eta / 2$ and $M_{\eta}=$ $\max _{1 \leq i \leq N} M_{\eta, i}$, where $\left\|V_{s, i}(0)\right\| \leq M_{\eta, i}$. Clearly, $\forall V(0)$, there is a $V_{s, i}(0)$ such that $\left\|V(0)-V_{s, i}(0)\right\|_{\beta} \leq \eta$, and we can apply Theorem 6.5 uniformly in $\tilde{B}_{\beta}$ on $[0, T]$.

Bootstrapping the previous corollary on a sequence of times $0, T, 2 T, \ldots, N T$ is possible, but yields the following exponential estimate for $\tilde{M}_{\beta}^{\prime}$ :

$$
\tilde{M}_{\beta}^{\prime}(N T) \leq \tilde{M}_{\beta}^{\prime}(T) \exp \left(N \log \left(C_{0}\right)\right),
$$

where $C_{0}$ is defined in (6.7). This prevents any straightforward construction of an absorbing ball in $H^{\beta}$, uniformly in $\alpha$. However, a weaker absorbing set can be constructed as the following:

Theorem 6.7. Let $0 \leq \alpha \leq \alpha_{M}, \nu>0$; let $a_{1}, a_{2}$, and $a_{3}$ be arbitrary and fixed. Let $\beta>5 / 2, \gamma>\beta+4$, and $F$ a time-independent force such that

$$
\|F\|_{\beta-1}^{2} \leq M_{\beta F}^{2} \text { and }\|F\|_{\gamma-1}^{2} \leq M_{\gamma F}^{2} .
$$

Let $V(0) \in B_{\gamma I}$ be initial data in a ball in $H^{\gamma}$. Let diam $\left(B_{\gamma I}\right)<2 \rho_{\gamma I}$ in $H^{\gamma}$ norm and $\operatorname{diam}\left(B_{\gamma I}\right)<2 \rho_{\beta I}$ in $H^{\beta}$ norm. Then, for each $f \geq f^{*}\left(M_{\beta F}, M_{\gamma F}, \rho_{\gamma I}, \rho_{\beta I}, \nu, a_{1}, a_{2}, a_{3}\right)$, the $3 D$ rotating Navier-Stokes- $\alpha$ equations possess an absorbing set $B_{\beta}$ in $H^{\beta}$; that is, there exists $t_{\beta}=t_{\beta}\left(\rho_{\beta I}\right)$, such that $f \geq f^{*}$ and $V(0) \in B_{\gamma I}$ imply

$$
\|V(t)\|_{\beta} \leq \rho_{\beta} \quad \text { and } \quad \nu \int_{t}^{t+1}\|V(\tau)\|_{\beta+1}^{2} d \tau \leq M_{\beta+1}^{2},
$$

for all $t \geq t_{\beta}$. This absorbing set is uniform in $\alpha$ and $\rho_{\beta}=\rho_{\beta}\left(M_{\beta F}, \nu, a_{1}, a_{2}, a_{3}\right)$, $M_{\beta+1}=M_{\beta+1}\left(M_{\beta F}, \nu, a_{1}, a_{2}, a_{3}\right)$ (with no dependence on $M_{\gamma F}$, nor on $\left.\rho_{\gamma I}\right)$.

Proof. We now choose $F$ smooth in $H^{\gamma}, \gamma>\beta+4, \beta>5 / 2$ and initial data in the smooth ball $\beta_{\gamma I}$ of radius $\rho_{\gamma I}$ in the $H^{\gamma}$ norm, and radius $\rho_{\beta I}$ in the $H^{\beta}$ norm. Of 
course, $H^{\gamma} \subset H^{\beta}$. We apply Theorem 6.3 with $\gamma=\sigma, M_{\sigma F}$ replaced by $\rho_{\gamma I}$ and $M_{\beta}$ replaced by $\rho_{\beta I}$. Then, for any $V(0) \in B_{\gamma I}$, we obtain regular solutions in the weaker topology of $H^{\beta}$, for $f \geq f^{*}\left(M_{\beta F}, M_{\gamma F}, \rho_{\beta I}, \rho_{\gamma I}, \nu, a_{1}, a_{2}, a_{3}\right)$.

$$
\|V(t)\|_{\beta} \leq M_{\beta}^{\prime}, 0 \leq t<\infty
$$

where $M_{\beta}^{\prime}=M_{\beta}^{\prime}\left(M_{\beta F}, \rho_{\beta I}, \nu, a_{1}, a_{2}, a_{3}\right)$ but does not depend on $M_{\gamma F}$ or $\rho_{\gamma I}$. We are using two deep (and subtle) features of Theorem 5.2 in [7]: the dependence of the threshold $f^{*}$ upon bounds in the highest norm $H^{\sigma}$ but the independence of $M_{\beta}^{\prime}$ from the latter; and that Theorem 5.2 in [7] can be extended to $T=\infty$. Then the proof of an absorbing ball for $B_{\gamma I}$ in the $H^{\beta}$ topology follows along classical lines, using the generalized (uniform) Gronwall lemma with norms in $H^{\beta}$ and $H^{\beta+1}$ (time average), together with finite regularization time in $H^{\beta+1}$, and we obtain $\rho_{\beta}, M_{\beta+1}$ independent from $\rho_{\gamma I}$ and $M_{\gamma F}$.

REMARK 6.8. Absorbing balls in a weaker topology than that of the set of initial data are a typical feature of infinite dimensional dynamical systems methods applied to damped hyperbolic PDE's (Ch6, [19]). This notion enables the construction of a global attractor as well as exponential attractors in the " $H^{\beta}-H^{\gamma}$ " sense ([28], Ch9). Here, of course, it is the lack of conservation of classical energy for the unfiltered $V_{\alpha}$ that creates the difficulty.

\section{Existence of global and exponential attractors, uniformly in $\alpha$}

In this section we follow the theory along the line of [19] to construct an exponential attractor for the 3D rotating Navier-Stokes- $\alpha$ equations for large $f$. We start out by recalling the procedure with which exponential attractors are constructed in Hilbert spaces.

Let $E$ be a Hilbert space with norm $\|\cdot\|_{E}$ induced by the inner product $(\cdot, \cdot)_{E}$. Let $X$ be a compact subset of $E$ and $S: X \rightarrow X$ a Lipschitz continuous map with Lipschitz constant $L$. Then $S$ possesses a global attractor $\mathcal{A}$ which is a compact, connected set given by

$$
\mathcal{A}=\bigcap_{n=1}^{\infty} S^{n}(X) .
$$

(Theorem 2.4.2, [23]). Exponential attractors for a map $S$ are defined as:

Definition 7.1. (Discrete Exponential Attractor) A compact set $\mathcal{M}$ is called an exponential attractor for $(S, X)$ if $\mathcal{A} \subset \mathcal{M} \subset X$ and

(i) (positively invariant) $S(\mathcal{M}) \subset \mathcal{M}$.

(ii) $\mathcal{M}$ has finite fractal dimension, $\operatorname{dim}_{F}(\mathcal{M})<\infty$.

(iii) There exist positive constants $c_{0}$ and $c_{1}$ such that

$$
d_{h}\left(S^{n} X, \mathcal{M}\right) \leq c_{0} e^{-c_{1} n}, \quad \forall n \geq 1
$$

where $d_{h}$ is the standard Hausdorff semi-distance between two sets defined by $d_{h}(A, B)=\sup _{a \in A} \inf _{b \in B}\|a-b\|_{E}$.

In establishing the existence of discrete exponential attractors key techniques are those based on examining the difference of two solutions and verifying the squeezing property on the underlying mapping $S$. The squeezing property guarantees the existence of discrete exponential attractors (Ch. 2, [19]): 
Definition 7.2. Let $E$ be a Hilbert space and $X$ a subset of $E$. A map $S$ has the squeezing property in $X$ if, for some $\delta \in\left(0, \frac{1}{4}\right)$, there exists an orthogonal projection $P=P(\delta)$ of finite rank $N_{0}=N_{0}(\delta)$ such that, $\forall u, v \in X$, if $\left\|\left(I-P_{N_{0}}\right)(S u-S v)\right\|_{E} \geq$ $\left\|P_{N_{0}}(S u-S v)\right\|_{E}$ then $\|S u-S v\|_{E} \leq \delta\|u-v\|_{E}$.

THEOREM 7.3. If $S$ has the squeezing property in $X$, then there exists an exponential attractor $\mathcal{M}$ for $(S, X)$ and, moreover,

$$
d_{B}(\mathcal{M}) \leq N_{0} \max \left\{1, \log \left(\frac{2 L}{\delta}+1\right) / \log \left(\frac{1}{\theta}\right)\right\}
$$

where $\theta \in(4 \delta, 1)$ arbitrary and $d_{B}$ is the fractal box dimension for the metric $E$.

We now turn to the continuous case. Let $\{S(t) \mid t \geq 0\}$ be the semigroup on $X$. Assume that $S(t)$ possesses a global attractor $\mathcal{A}$. The exponential attractors for $(S(t), X)$ are defined as:

Definition 7.4. (Continuous Exponential Attractor) A compact set $\mathcal{M}$ is called an exponential attractor for a semigroup $(S(t), X)$ if

(i) $\mathcal{A} \subseteq \mathcal{M} \subseteq X$

(ii) $S(t) \mathcal{M} \subseteq \mathcal{M}$ for $t \geq 0$,

(iii) the fractal dimension of $\mathcal{M}$ is finite, $\operatorname{dim}_{F}(\mathcal{M})<\infty$,

(iv) there exists positive constants $c_{0}$ and $c_{1}$ such that

$$
d_{h}(S(t) X, \mathcal{M}) \leq c_{0} e^{-c_{1} t}, \forall t \geq 0,
$$

where $d_{h}$ is the Hausdorff semi-distance for the metric E.

Given the semigroup $\{S(t) \mid t \geq 0\}$ of solution operators, we will choose a positive $t_{*}$ small enough such that $S_{*}=S\left(t_{*}\right)$ possesses the squeezing property in $X$. If $S_{*}$ is Lipschitz continuous, then the existence of a discrete exponential attractor $\mathcal{M}_{*}$ for $\left(S_{*}, X\right)$ is guaranteed by Theorem 7.3. Next we define

$$
\mathcal{M}=\bigcup_{0 \leq t \leq t_{*}} S(t) \mathcal{M}_{*}
$$

and $G:[0, T] \times \mathcal{M}_{*} \rightarrow \mathcal{M}$ as $G(t, x)=S(t) x$. If $G$ is Lipschitz, then it can be shown that $\mathcal{M}$ is a compact set with a finite fractal box dimension, and $\mathcal{M}$ will be an exponential attractor for $(S(t), X)$ (Theorem 3.1, [19]). The exponential attractors for the continuous dynamical systems generated by a semigroup $\{S(t)\}_{t \geq 0}$ are unions of exponential attractors restricted by squeezing time $t_{*}$. In addition, given an estimate for $\mathcal{M}_{*}$, it is not difficult to get an estimate for the fractal box dimension of $\mathcal{M}$ (Theorem 3.1, [19]):

THEOREM 7.5. Let $S\left(t_{*}\right)$ have the squeezing property in $X$ for some time $t_{*}>0$ and let $\mathcal{M}$ be an exponential attractor for $(S(t), X)$ and $G\left(t_{*}, x\right)=S\left(t_{*}\right) x$ for $x \in X, t \geq 0$. If $G\left(t_{*}, \cdot\right)$ is Lipschitz in $X$ with Lipschitz constant $L_{*}$, then

$$
d_{B}(\mathcal{M}) \leq d_{B}\left(\mathcal{M}_{*}\right)+1
$$

Furthermore,

$$
d_{h}(S(t) X, \mathcal{M}) \leq c L_{*} \exp \left(\frac{-(\ln 8) t}{t_{*}}\right)
$$


for all $t \geq 0$, where $c$ is a positive constant.

Now we follow the above procedure to establish the existence of an exponential attractor in $L^{2}$ for the 3D RNS- $\alpha$ equations. We do this for all $f$ that allow the existence of a global attractor. Assume that $F$ is time-independent and smooth and that $f \geq f^{*}$ as in Th 6.7. Let $S_{\alpha}(t)$ be the semiflow for solutions of the 3D RNS $\alpha$-equations and let $B_{\beta}, \beta>5 / 2$, be the absorbing ball obtained in Theorem 6.7 . Set

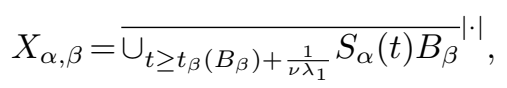

where the closure is taken in $L^{2}$-topology and $\lambda_{1}$ denotes the first eigenvalue of the Stokes operator. Then $X_{\alpha, \beta}$ is a bounded subset of $B_{\beta}$, compact in $H^{s}, 0 \leq s<\beta$, and positively invariant under $S_{\alpha}(t)$ such that, for all $V_{\alpha}(0) \in X_{\alpha, \beta}$,

$$
\left\|S_{\alpha}(t) V_{\alpha}(0)\right\|_{H^{\beta}} \leq \rho_{\alpha, \beta}, \quad \forall t \geq 0
$$

where $\rho_{\alpha, \beta}$ is the uniform bound obtained in Th 6.7. In particular, there exist absolute bounds $\rho_{\alpha, s}=\rho_{\alpha, s}\left(M_{\beta F}, \nu, a_{1}, a_{2}, a_{3}\right)$ such that $\left\|S_{\alpha}(t) V_{\alpha}(0)\right\|_{H^{s}} \leq \rho_{\alpha, s} \leq \rho_{\alpha, \beta}$ for $0 \leq$ $s<\beta$. We will denote $\rho_{H}$ for $\rho_{\alpha, 0}$ and $\rho_{V}$ for $\rho_{\alpha, 1}$. Since $X_{\alpha, \beta}$ is compact in $H^{s}$ for $0 \leq s<\beta$, we can deduce that the underlying semigroup $S(t)$ is uniformly compact so that it possesses a unique global attractor $\mathcal{A}$ in $H^{s}$ for $0 \leq s<\beta$, (Theorem 1.1, [37]). Moreover, it can be proved that $\mathcal{A}$ lies in $H^{\beta}$ for $\beta>5 / 2$, (Corollary 9.7, [28]).

We consider the solution operator $S_{\alpha}(t)$ as a map from $X_{\alpha, \beta}$ into $X_{\alpha, \beta}$. We only need to show that there exists a squeezing time $t_{*}$ such that the discrete operator $S_{\alpha *}=S_{\alpha}\left(t_{*}\right)$ has the squeezing property in $L^{2}$-topology. To achieve it we first examine the difference between two solutions, $V_{a}$ and $V_{b}$, of 3D RNS- $\alpha$ equations in $X_{\alpha, \beta}$. Let $W=V_{a}-V_{b}$ and $W^{\prime}=\frac{V_{a}+V_{b}}{2}$. Then $W$ satisfies the equation

$$
\begin{gathered}
\frac{\partial W}{\partial t}+\nu A W+f M \mathcal{R}_{\alpha} W=-\left[B_{\alpha}\left(W^{\prime}, W\right)+B_{\alpha}\left(W, W^{\prime}\right)\right] \\
W(0)=V_{a}(0)-V_{b}(0) .
\end{gathered}
$$

Taking the inner product with $2 W$ and using Lemma 2.3 we obtain

$$
\frac{d}{d t}|W|^{2}+2 \nu\|W\|^{2} \leq 2\left\{\left|<B_{\alpha}\left(W^{\prime}, W\right), W>\right|+\left|<B_{\alpha}\left(W, W^{\prime}\right), W>\right|\right\},
$$

where $B_{\alpha}(u, v)=\left(\mathcal{R}_{\alpha} u \cdot \nabla\right) v+v_{j} \nabla\left(\mathcal{R}_{\alpha} u\right)_{j}$. Estimating the right hand side of (7.3) and using Young's inequality yields

$$
\frac{d}{d t}|W|^{2}+\nu\|W\|^{2} \leq \frac{K_{1}}{\nu^{3}}|W|^{2}
$$

where $K_{1}=c_{1}^{4} \rho_{V}^{4}$ with $c_{1}$ a constant. Letting $\lambda(t)=\frac{\|W(t)\|^{2}}{|W(t)|^{2}},(7.4)$ becomes

$$
\frac{d}{d t}\left[\ln |W(t)|^{2}\right] \leq-\nu \lambda(t)+\frac{K_{1}}{\nu^{3}}
$$

so that

$$
|W(t)|^{2} \leq \delta(t)|W(0)|^{2}
$$

with

$$
\delta(t)=\exp \left(-\nu \int_{0}^{t} \lambda(s) d s+\frac{K_{1}}{\nu^{3}} t\right) .
$$


Next, we need to find a time $t_{*}$ such that the estimate for $\delta\left(t_{*}\right)$ allows squeezing. Thus it is essential to bound $\int_{0}^{t_{*}} \lambda(s) d s$, and following the exact line of section 6.1 in [38] we obtain

$$
t_{*}=\frac{c_{3}^{2}}{c_{2}} \frac{\nu^{3 / 2}}{K_{2} K_{3}}
$$

where $K_{2}=c_{2} \rho_{V}$ and $K_{3}^{2}=\frac{27 c_{3}^{4}}{2 \nu^{3}} \rho_{V}^{6}+\frac{2}{\nu \lambda_{1}} \rho_{H}$ with $c_{2}$ and $c_{3}$ constants. Furthermore,

$$
\int_{0}^{t_{*}} \lambda(t) d t \geq c_{4} \lambda_{N_{0}+1} \frac{\nu^{3 / 2}}{K_{2} K_{3}}
$$

where $c_{4}=\frac{1}{2}\left[1-\exp \left(-c_{3}^{2} / c_{2}\right)\right]>0$, so that

$$
\delta\left(t_{*}\right) \leq \exp \left(-\frac{c_{4}}{c_{2}} \lambda_{N_{0}+1} \frac{\nu^{5 / 2}}{K_{3} \rho_{V}}+\frac{c_{5} \rho_{V}^{3}}{\nu^{3 / 2} K_{3}}\right),
$$

where $c_{5}=\frac{27}{16} c_{1}^{4} c_{3}^{2} c_{2}^{2}$. By the definition of $K_{3}$ there exists a constant $\tilde{c}>0$ such that

$$
K_{3} \leq \tilde{c}\left(\frac{\rho_{V}^{3}}{\nu^{3 / 2}}+\nu^{1 / 2} \lambda_{1}^{1 / 2} \rho_{H}\right)
$$

Choosing $N_{0}$ such that

$$
N_{0} \geq \tilde{c}^{3 / 2} \max \left\{\frac{1}{\lambda_{1}^{3 / 4}} \frac{\left(\rho_{H} \rho_{V}\right)^{3 / 2}}{\nu^{3}}, \frac{\rho_{V}^{6}}{\lambda_{1}^{3 / 2} \nu^{6}}\right\},
$$

gives $\delta\left(t_{*}\right)<\frac{1}{8}$. Under the above condition of $N_{0}$, the following Lemma assures the existence of an exponential attractor $\mathcal{M}_{\alpha}^{*}$ for $\left(S_{*}, X_{\alpha, \beta}\right)$ for $f \geq f_{*}$ (Ch 3, [19]; Proposition 2.2.7, [38]):

Lemma 7.6. Let $t_{*}>0$ be given and $u, v \in X$. Define

$$
\lambda_{*}=\frac{\left\|w_{*}\right\|^{2}}{\left|w_{*}\right|^{2}},
$$

where $w_{*}=S_{*} u-S_{*} v$. Then $S_{*}$ possesses the squeezing property in $X$, if there exists $\delta \in(0,1 / 4)$ and $N_{0}=N_{0}(\delta) \in \mathcal{N}$, such that $\lambda_{*}>\frac{1}{2} \lambda_{N_{0}+1}$ implies that $\left|S_{*} u-S_{*} v\right|<$ $\delta|u-v|$, for all $u, v \in X$.

Furthermore, the Lipschitz constant for $S_{*}$ on $X_{\alpha, \beta}$ is estimated as

$$
L_{*}=\delta\left(t_{*}\right) \leq \exp \left(\frac{c_{5} \rho_{V}^{3}}{\nu^{3 / 2} K_{3}}\right),
$$

and hence

$$
\begin{aligned}
d_{h}\left(S_{\alpha}(t) X_{\alpha, \beta}, \mathcal{M}_{\alpha}^{*}\right) & \leq c L_{*}\left(\left(\delta\left(t_{*}\right)\right)^{1 / t_{*}}\right)^{t} \\
& \leq c L_{*}\left(e^{-\ln 8}\right)^{t / t_{*}} \\
& =c_{\alpha F} e^{-\delta_{\alpha F} t}
\end{aligned}
$$


where $c_{\alpha F}=c L_{*}$ and $\delta_{\alpha F}=\frac{\ln 8}{t_{*}}$.

Now we summarize the results:

THEOREM 7.7. Let $F$ be a smooth, time-independent force and let $a=\left(a_{1}, a_{2}, a_{3}\right)$ be a domain size parameter. For $f \geq f_{*}$ as in Th 6.7, let $X_{\alpha, \beta}$ be the positively invariant set from (7.1). Then $\left\{S_{\alpha}(t) \mid t \geq 0\right\}$ restricted to $X_{\alpha, \beta}$ admits an exponential attractor $\mathcal{M}_{\alpha}$ in $L^{2}$. Moreover, the rate of convergence to the exponential attractor is given by

$$
d_{h}\left(S_{\alpha}(t) X_{\alpha, \beta}, \mathcal{M}_{\alpha}\right) \leq c_{\alpha F} e^{-\delta_{\alpha F} t},
$$

where $c_{\alpha F}, \delta_{\alpha F}$ are constants, which only depend on $\nu, a, \rho_{\alpha H}, \rho_{\alpha V}$ and are independent of the angular frequency $f \geq f_{0}$ and $\alpha>0$.

REMARK 7.8. $\mathcal{M}_{\alpha}$ is bounded in $H^{\beta}$ and attracts all orbits in the $L^{2}$-norm topology. It is compact in the space $H^{\gamma}, 0 \leq \gamma<\beta$.

\section{Continuity of exponential attractors}

In this section we investigate the convergence of the exponential attractor $\mathcal{M}_{\alpha, \beta}$ for the semiflow $S_{\alpha}(t)$ to the one for the semiflow $S_{0}(t)$ of the original RNSE as $\alpha$ vanishes by establishing the upper and lower semicontinuity of the exponential attractors with respect to the Hausdorff semi-distance, as $\alpha \rightarrow 0^{+}$. In the previous section, we constructed the exponential attractor $\mathcal{M}_{\alpha, \beta}$ for the 3D RNS- $\alpha$ equations when $\alpha>0$. Now we need the corresponding exponential attractor for the original RNSE (when $\alpha=0$ ). Trahe ([38]) constructed an exponential attractor $\mathcal{M}_{f}$ for the original RNSE. We extend his attractor to the space $H^{\beta}$.

8.1. Construction of exponential attractors in $H^{\beta}$ for 3D RNSE. Trahe ([38]) constructed an exponential attractor $\mathcal{M}_{f}$ for the 3D RNSE with a smooth, time-independent force $F$ for all $f$ large enough. His construction of the exponential attractor is based on the existence and global regularity theorems of solutions to the 3D RNSE ([7]):

TheOREM 8.1. Let the domain parameters $a=\left(a_{1}, a_{2}, a_{3}\right)$ be fixed but arbitrary. Let $\nu>0, s>1 / 2$, and $F$ a smooth time-independent force with $\|F\|_{s-1} \leq M_{s, F}$. Let $\|V(0)\|_{s} \leq \tilde{M}_{s}$. Then there exists $f_{s}=f_{s}\left(\tilde{M}_{s}, M_{s, F}, \nu, a\right)$ such that for each $f \geq f_{s}$, the corresponding solutions to the $3 D R N S E(\alpha=0)$ are regular for all $t \geq 0$ and

$$
\|V(t)\|_{s} \leq \tilde{M}_{s}^{\prime}, \quad \forall t \geq 0,
$$

where $\tilde{M}_{s}^{\prime}=\tilde{M}_{s}^{\prime}\left(\tilde{M}_{s}, M_{s, F}, \nu, a\right)$

THEOREM 8.2. Let the domain parameters $a=\left(a_{1}, a_{2}, a_{3}\right)$ be fixed but arbitrary. Let $\nu>0, s>1 / 2$, and $F$ a smooth time-independent force with $\|F\|_{s-1} \leq M_{s, F}$. Let $\|V(0)\|_{0} \leq \tilde{M}_{0}$. Then there exist a time $\hat{T}=\hat{T}\left(M_{0}, M_{s F}, \nu\right)$ and $f^{\prime}$ such that, for each $f \geq f^{\prime}$, the corresponding weak Leray solution $V(t)$ of the $3 D$ RNSE $(\alpha=0)$ defined on $[0, \hat{T}]$ satisfies the following: $V$ can be extended to $(0, \infty)$ and is regular for each $t \in[\hat{T}, \infty)$, i.e., $V$ belongs to $H^{s}$ and $\|V(t)\|_{s} \leq C\left(a, M_{s F}, \nu\right)$ for all $t \geq \hat{T}$.

This theorem describes the situation when $f$ is fixed and large enough depending only on the magnitude of $F$ and independent of nonsmooth initial data $V(0) \in L^{2}$. Then weak Leray solutions $V(t)$ are always regular for $t>\hat{T}$.

Using these two theorems Trahe established the existence of absorbing balls in $H^{1}$ as well as in $L^{2}$ (Section 6.1, [38]). 
Proposition 8.3. Let $F$ be a time-independent, smooth force. Then for each $f \geq 0$, there exists an absorbing set $B_{H}$ in $L^{2}$, independent of $f$, for every weak Leray solution of the $3 D$ RNSE. That is, given a bounded set $B$ in $L^{2}$, there exists $t_{H}=t_{H}(B)$, such that each $V(0) \in B$ implies

$$
\|V(t)\|_{0}=\left\|S_{0}(t) V(0)\right\|_{0} \leq \rho_{H}=\sqrt{2} \frac{\|F\|_{0}}{\nu \lambda_{1}}
$$

for all $t \geq t_{H}$.

Proposition 8.4. Let $F$ be a time independent, smooth force with $\|F\|_{0} \leq M_{1, F}$. Let $M_{0}=M_{0}\left(1 /\left(\nu \lambda_{1}\right)\right)$ and $\tilde{M}_{1}=\sqrt{2 M_{0}}$, where $\tilde{M}_{1}$ is from the hypothesis of Theorem 8.1 with $s=1$. Then, for each $f \geq f_{0}=\max \left\{f^{\prime}, f_{1}\right\}$, the $3 D$ RNSE possess an absorbing set $B_{V}$ in $H^{1}$; that is, given $B \subseteq H^{1}$ bounded, there exists $t_{V}=t_{V}(B)$ such that $f \geq f_{0}$ and $V(0) \in B$ imply

$$
\|V(t)\|_{1}=\left\|S_{0}(t) V(0)\right\|_{1} \leq \rho_{V}
$$

for all $t \geq t_{V}$. Here $\rho_{V}$ is a constant depending on $\rho_{H}, \nu, a_{1}, a_{2}, a_{3}$.

REMARK 8.5. Trahe also constructed the absorbing set $B_{W}$ in $H^{2}$, which has size of $\rho_{W}=\rho_{W}\left(\rho_{h}, \nu, a_{1}, a_{2}, a_{3},\|F\|_{1}\right)$. The absorbing set and the entry time are uniform for all $f \geq f_{0}$.

We extend this result to get an absorbing ball $B_{0, \beta}$ in space $H^{\beta}$ via the usual 3D NSE smoothing process:

Proposition 8.6. Under the same hypothesis as in Proposition 8.4, for each $f \geq f_{0}=$ $\max \left\{f^{\prime}, f_{1}\right\}$, the $3 D R N S E$ possess an absorbing set $B_{0, \beta}$ in $H^{\beta}$; i.e., given $B \subseteq H^{\beta}$ bounded, there exists $t_{\beta}=t_{0, \beta}(B) \geq t_{V}\left(B_{V}\right)+\frac{1}{\nu \lambda_{1}}$ such that $f \geq f_{0}$ and $V(0) \in B$ imply

$$
\|V(t)\|_{\beta}=\left\|S_{0}(t) V(0)\right\|_{\beta} \leq \rho_{0, \beta}
$$

for all $t \geq t_{0, \beta}$.

Define

$$
X_{0, \beta}=\bigcup_{t \geq t_{0, \beta}\left(B_{0, \beta}\right)+\frac{1}{\nu \lambda_{1}}} S_{0}(t) B_{0, \beta} \cdot \mid
$$

where the closure is taken in the $L^{2}$-norm topology. Then $X_{0, \beta}$ is a bounded subset of $B_{0, \beta}$ and is compact in the spaces $H^{\sigma}, 0 \leq \sigma<\beta$. In particular, if $V(0) \in X_{0, \beta}$,

$$
\left|S_{0}(t) V(0)\right| \leq \rho_{H},\left\|S_{0}(t) V(0)\right\| \leq \rho_{V} \text {, and }\left\|S_{0}(t) V(0)\right\|_{2} \leq \rho_{W}, \forall t \geq 0 .
$$

Let $\mathcal{M}_{f}$ be an exponential attractor in $H^{0}$ constructed in Ch. 6, [38]. We simply restrict it to the set $X_{0, \beta}$ to obtain an exponential attractor $\mathcal{M}_{0, \beta}=\mathcal{M}_{f} \cap X_{0, \beta}$.

Proposition 8.7. Let the domain parameters $a=\left(a_{1}, a_{2}, a_{3}\right)$ be fixed but arbitrary, $F$ a smooth time-independent force, and $X_{0, \beta}$ a positively invariant compact set from (8.1). Then, for each $f \geq f_{0},\left\{S_{0}(t)\right\}$ restricted to $X_{0, \beta}$ admits an exponential attractor $\mathcal{M}_{0, \beta}$. Moreover, the rate of convergence to the exponential attractor is given by

$$
d_{h}\left(S_{0}(t) X_{0, \beta}, \mathcal{M}_{0, \beta}\right) \leq c_{0 F} e^{-\delta_{0 F} t},
$$

where $c_{f}, \delta_{F}$ are constants, which only depend on $\nu, a, \rho_{H}, \rho_{V}$ and are independent of the angular frequency $f \geq f_{0}$.

Remark 8.8. In Proposition 8.7, the Hausdorff semi-distance is defined with $L^{2}$ norm. 
8.2. Convergence of exponential attractors. Now we express Eq. (1.1) in the equivalent form that is very useful in showing the continuity of exponential attractors. Rewrite Eq. (1.1) as

$$
\frac{\partial V}{\partial t}+\left(\mathcal{R}_{\alpha} V \cdot \nabla\right) V+V_{j} \nabla\left(\mathcal{R}_{\alpha} V\right)_{j}+f J \mathcal{R}_{\alpha} V+\nu A V=-\nabla \pi+F .
$$

Notice that

$$
\begin{aligned}
V_{j} \nabla\left(\mathcal{R}_{\alpha} V\right)_{j} & =V_{j} \nabla\left(\left[\mathcal{R}_{\alpha}-\mathrm{I}\right] V\right)_{j}+V_{j} \nabla V_{j} \\
& =V_{j} \nabla\left(\left[\mathcal{R}_{\alpha}-\mathrm{I}\right] V\right)_{j}+\frac{1}{2} \nabla\left|V_{j}\right|^{2},
\end{aligned}
$$

where $I$ is the identity matrix. Since $P_{L}\left[\frac{1}{2} \nabla\left|V_{j}\right|^{2}\right]=0$, Eq.(1.1) becomes, after Leray projection,

$$
\frac{\partial V}{\partial t}+\nu A V+P_{L}\left[\left(\mathcal{R}_{\alpha} V \cdot \nabla\right) V\right]+P_{L}\left[V_{j} \nabla\left(\left[\mathcal{R}_{\alpha}-\mathrm{I}\right] V\right)_{j}\right]+f P_{L} J P_{L} \mathcal{R}_{\alpha} V=F
$$

The operator $\mathcal{R}_{\alpha}-I$ in Eq.(8.2) is crucial in proving the convergence of solutions of the 3D RNS- $\alpha$ equations because its norm in Fourier-Sobolev spaces may be taken as small as needed:

Lemma 8.9. Let $0 \leq s<\infty$ and $\beta>0$ be given. For any arbitrary small number $\epsilon>0$, there exists a constant $\tilde{\alpha}(\epsilon)$ such that, $\forall 0<\alpha \leq \tilde{\alpha}(\epsilon)$,

$$
\left\|\left(\mathcal{R}_{\alpha}-I\right) v\right\|_{H^{s}}^{2} \leq \epsilon\|v\|_{H^{s+\beta}}^{2}, \quad \text { for any } v \in H^{s+\beta} .
$$

In particular, $\tilde{\alpha}(\epsilon) \rightarrow 0$ as $\epsilon \rightarrow 0$. $M>0$,

Proof. Note that, for any wave number $k,\left(\mathcal{R}_{\alpha}-I\right)_{k}=\frac{-\alpha^{2}|\check{k}|^{2}}{1+\alpha^{2} \mid \breve{k}^{2}}$. Then, for any

$$
\begin{aligned}
\left\|\left(\mathcal{R}_{\alpha}-I\right) v\right\|_{H^{s}}^{2} & =\sum_{k}\left[\frac{\alpha^{2}|\check{k}|^{2}}{1+\alpha^{2}|\check{k}|^{2}}\right]^{2}|\check{k}|^{2 s}\left|v_{k}\right|^{2} \\
& \leq \sum_{k} \frac{\alpha^{4}|\check{k}|^{4}}{1+\alpha^{4}|\check{k}|^{4}}|\check{k}|^{2 s}\left|v_{k}\right|^{2} \\
& \leq \sum_{|k|<M} \frac{\alpha^{4}|\check{k}|^{4}}{1+\alpha^{4}|\check{k}|^{4}}|\check{k}|^{2 s}\left|v_{k}\right|^{2}+\sum_{|k| \geq M}|\check{k}|^{2 s}\left|v_{k}\right|^{2} .
\end{aligned}
$$

Now:

(i)

$$
\begin{aligned}
\sum_{|k| \geq M}|\check{k}|^{2 s}\left|v_{k}\right|^{2} & =\sum_{|k| \geq M} \frac{1}{|\check{k}|^{2 \beta}}|\check{k}|^{2(s+\beta)}\left|v_{k}\right|^{2} \\
& \leq \frac{1}{M^{2 \beta}}\|v\|_{H^{s+\beta}}^{2} .
\end{aligned}
$$

(ii) Choose the closest integer $\check{M}$ to $\left(\frac{2}{\epsilon}\right)^{\frac{1}{2 \beta}}$ satisfying $\check{M}=\left\lceil\left(\frac{2}{\epsilon}\right)^{\frac{1}{2 \beta}}\right\rfloor+1$, where $\lceil\cdot 」$ denotes the integer part of "." ". Then $\frac{1}{\dot{M}^{2 \beta}} \leq \frac{\epsilon}{2}$, and, from (i), we obtain

$$
\sum_{|k| \geq \tilde{M}}|\check{k}|^{2 s}\left|v_{k}\right|^{2} \leq \frac{\epsilon}{2}\|v\|_{H^{s+\beta}}^{2} .
$$


(iii) Now choose $\tilde{\alpha}(\epsilon)=\left(\frac{\epsilon}{2}\right)^{\frac{1}{2 \beta}}\left(\frac{\epsilon / 2}{1-\epsilon / 2}\right)^{1 / 4}$. Then

$$
\alpha^{4}<\tilde{\alpha}^{4}(\epsilon) \approx \frac{1}{\check{M}^{4}} \frac{\epsilon / 2}{1-\epsilon / 2}
$$

It implies $\alpha^{4} \check{M}^{4} \leq \frac{\epsilon / 2}{1-\epsilon / 2}$ so that $\frac{\alpha^{4} \check{M}^{4}}{1+\alpha^{4} \check{M}^{4}} \leq \frac{\epsilon}{2}$. Thus

$$
\sum_{|k|<\check{M}} \frac{\alpha^{4} \check{M}^{4}}{1+\alpha^{4} \check{M}^{4}}|\check{k}|^{2 s}\left|v_{k}\right|^{2} \leq \frac{\epsilon}{2}\|v\|_{H^{s}}^{2} \leq \frac{\epsilon}{2}\|v\|_{H^{s+\beta}}^{2} .
$$

Combining (i)-(iii) with the inequality (8.4), we get, for any $0<\alpha \leq \tilde{\alpha}(\epsilon)$,

$$
\left\|\left(\mathcal{R}_{\alpha}-I\right) v\right\|_{H^{s}}^{2} \leq \epsilon\|v\|_{H^{s+\beta}}^{2}, \quad \text { for any } v \in H^{s+\beta} .
$$

This lemma can be generalized to the following lemma, using the Hölder's inequality and choosing the closest integer $\check{M}$ to $\frac{2}{\epsilon}$ in part (i) and (ii) in the proof of Lemma 8.9, respectively:

Lemma 8.10. Let $0 \leq s<\infty$ and $\beta>0$ be given. For any arbitrary small number $\epsilon>0$, there exists a constant $\tilde{\alpha}(\epsilon)$ such that, $\forall 0<\alpha \leq \tilde{\alpha}(\epsilon)$,

$$
\left\|\left(\mathcal{R}_{\alpha}-I\right) v\right\|_{H^{s}} \leq C(\beta) \epsilon^{2 \beta /(\beta+2)}\|v\|_{H^{s}}^{\beta /(\beta+2)}\|v\|_{H^{s+\beta}}^{2 /(\beta+2)}, \quad \text { for any } v \in H^{s+\beta} .
$$

In particular, $\tilde{\alpha}(\epsilon) \rightarrow 0$ as $\epsilon \rightarrow 0$.

Now we prove the continuity of exponential attractors:

THEOREM 8.11. Let $\nu>0, a=\left(a_{1}, a_{2}, a_{3}\right)$ fixed, and $F$ a smooth time-independent force. Let $f_{*}$ fixed, given in the existence theorem 6.7. Let $\eta>0$ given. Then there exist time $\tilde{t}=\tilde{t}\left(\eta, \nu, \rho_{0, \beta}, \rho_{\alpha, \beta}, a\right)>0$ and $\tilde{\alpha}\left(\eta, \nu, M_{\beta, F}, f_{*}\right)$ such that, for $0<\alpha \leq \tilde{\alpha}$,

(i) $d_{h}\left(S_{\alpha}(\tilde{t}) \mathcal{M}_{\alpha, \beta}, \mathcal{M}_{0, \beta}\right)<\eta$

(ii) $d_{h}\left(S_{0}(\tilde{t}) \mathcal{M}_{0, \beta}, \mathcal{M}_{\alpha, \beta}\right)<\eta$.

For a given $f_{*}$ this convergence is uniform in $\alpha, 0<\alpha<\tilde{\alpha}$. Moreover, $\tilde{\alpha} \rightarrow 0$ as $\eta \rightarrow 0$.

Proof. Proof of (i): Let $\eta>0$. We need to show that there exist $\tilde{t}=\tilde{t}(\eta)$ and $\tilde{\alpha}=\tilde{\alpha}(\eta)$ such that

$$
\forall x \in S_{\alpha}(\tilde{t}) \mathcal{M}_{\alpha, \beta} \quad \exists y \in \mathcal{M}_{\alpha, \beta} \text { such that }|x-y|<\eta .
$$

Since $S_{\alpha}(\tilde{t}) \mathcal{M}_{\alpha, \beta} \subseteq \mathcal{M}_{\alpha, \beta} \cap S_{\alpha}(\tilde{t}) \mathcal{M}_{\alpha, \beta}$, it suffices to show this for $x \in \mathcal{M}_{\alpha, \beta} \cap$ $S_{\alpha}(\tilde{t}) \mathcal{M}_{\alpha, \beta}$.

First we determine such $\tilde{t}$. Let $t_{b}=t_{b}\left(B_{\alpha, \beta}\right)$ be the entry time for the set $B_{\alpha, \beta}$ for the solutions to $3 \mathrm{D}$ RNS- $\alpha$ equations into the absorbing set $B_{0, \beta}$ for the solutions to the 3D RNSE under the semiflow $\left\{S_{0}(t)\right\}$. Recall that $B_{\alpha, \beta}$ is itself absorbing under $\left\{S_{\alpha}(t)\right\}$. It is clear that, $\forall t \geq t_{b}+t_{0, \beta}+\frac{1}{\nu \lambda_{1}}, S_{0}(t) B_{0, \beta} \subseteq X_{0, \beta}$. By Proposition 8.7, we can pick $\tilde{t} \geq t_{b}+t_{2}+\frac{1}{\nu \lambda_{1}}$ such that

$$
d_{h}\left(S_{0}(\tilde{t}) B_{0, \beta}, \mathcal{M}_{0, \beta}\right)<c_{0 F} e^{-\delta_{0 F}\left(\tilde{t}-\left(t_{b}+t_{\beta}+\frac{1}{\nu \lambda_{1}}\right)\right)}<\frac{\eta}{2} .
$$


Notice that $\tilde{t}$ depends on $\eta, t_{b}, t_{2}, \delta_{0 F}, c_{0 F}, \nu$. This implies that, given $\eta>0, \exists y \in \mathcal{M}_{0, \beta}$ such that

$$
\left|S_{0}(t) V(0)-y\right|<\frac{\eta}{2} \text { for every } V(0) \in B_{0, \beta} .
$$

Now suppose $x \in \mathcal{M}_{\alpha, \beta} \cap S_{\alpha}(\tilde{t}) \mathcal{M}_{\alpha, \beta}$. Then,

$$
\exists V(0) \in X_{\alpha, \beta} \text { such that } x=S_{\alpha}(\tilde{t}) V(0) .
$$

Thus we have

$$
|x-y| \leq\left|S_{\alpha}(\tilde{t}) V(0)-S_{0}(\tilde{t}) V(0)\right|+\left|S_{0}(\tilde{t}) V(0)-y\right| .
$$

From the tracking Lemma 8.12 below with $T=\tilde{t}(\eta), B_{0,1}=X_{0, \beta}, B_{\alpha, \beta}=X_{\alpha, \beta}, T_{*}=t_{b}+$ $t_{\beta}+\frac{1}{\nu \lambda_{1}}$, and $t_{1}=$ the initial time, we obtain, for $\alpha$ small enough,

$$
\left|S_{\alpha}(\tilde{t}) V(0)-S_{0}(\tilde{t}) V(0)\right|<\frac{\eta}{2}
$$

. This completes the proof. Proof of (ii) follows the same procedure.

Lemma 8.12. Let $[0, T]$ be a time interval on which the solutions of the $3 D$ RNSE and the $3 D R N S-\alpha$ equations are in respective absorbing sets in $B_{0,1}$ in $H^{1}$, with the radius of $M_{1}^{\prime}$, and $B_{\alpha, \beta}$ in $H^{\beta}, \beta>5 / 2$, with the radius of $M_{\beta}^{\prime}$. Assume $f \geq f^{*}$, where $f^{*}$ is defined in Theorem 6.7. Consider two solutions, $V_{0}$ and $V_{\alpha}$, of the $3 D$ rotating Navier-Stokes equations and the $3 D$ rotating Navier-Stokes- $\alpha$ equations, respectively, with the same initial data at some time $t_{1} \geq T_{*}$, where $T_{*}=\max \left\{T_{\alpha 0}, T_{0}\right\}, T_{\alpha 0}=$ the initial time when $V_{\alpha}(t)$ enters into an absorbing set, and $T_{0}=$ the initial time when $V_{0}(t)$ enters into an absorbing set. Then two solutions uniformly converge as $\alpha \rightarrow 0$ in $L^{2}$ for all $t \in\left[t_{1}, T\right]$ in the following sense: For any $\eta>0, \exists \tilde{\alpha}\left(\eta, \frac{1}{f}, T, M_{\beta}^{\prime}, M_{1}^{\prime}\right)$ such that, for any $\alpha<\tilde{\alpha}\left(\eta, \frac{1}{f}, T, M_{\beta}^{\prime}, M_{1}^{\prime}\right)$,

$$
\left\|V_{\alpha}(t)-V_{0}(t)\right\|_{0} \leq \eta, \quad \forall t \in\left[t_{1}, T\right], t_{1} \geq T_{*},
$$

and, for fixed $f \geq f^{*}, \eta \rightarrow 0$ as $\tilde{\alpha} \rightarrow 0$. The limit is uniform in $f$ for $f^{*} \leq f \leq f_{\max }$. Moreover, $\tilde{\alpha}\left(\eta, \frac{1}{f}, T, M_{\beta}^{\prime}, M_{1}^{\prime}\right) \rightarrow 0$ as $\eta \rightarrow 0$ and $\frac{1}{f} \rightarrow 0$.

Proof. Let $V_{0}$ and $V_{\alpha}$ be two solutions of the 3D rotating Navier-Stokes equations and the $3 \mathrm{D}$ rotating Navier-Stokes- $\alpha$ equations, respectively. Let $B_{0,1}$ be an absorbing set for $V_{0}$ in $H^{1}$ and let $B_{\alpha, \beta}, \beta>5 / 2$ be an absorbing set for $V_{\alpha}$ in $H^{\beta}$. We assume that $V_{0}$ is in $B_{0,1}$ and $V_{\alpha}$ is in $B_{\alpha, \beta}$ with:

$$
\left\|V_{0}\right\| \leq M_{1}^{\prime},\left\|V_{\alpha}\right\|_{\beta} \leq M_{\beta}^{\prime},
$$

where $M_{\beta}^{\prime}=\rho_{\beta}$ in Theorem 6.7. Let $T$ be an arbitrary, but fixed time. In addition, along with the existence results from [7], we will assume that $f$ is large and finite. The corresponding equations are

$$
\begin{gathered}
\frac{\partial V_{\alpha}}{\partial t}+\nu A V_{\alpha}+P_{L}\left[\left(\mathcal{R}_{\alpha} V_{\alpha} \cdot \nabla\right) V_{\alpha}\right]+P_{L}\left[V_{\alpha j} \nabla\left(\left\{R_{\alpha}-I\right\} V_{\alpha}\right)_{j}\right]+f P_{L} J P_{L} \mathcal{R}_{\alpha} V_{\alpha}=F \\
\frac{\partial V_{0}}{\partial t}+\nu A V_{0}+P_{L}\left[\left(V_{0} \cdot \nabla\right) V_{0}\right]+f P_{L} J P_{L} V_{0}=F .
\end{gathered}
$$


Writing $Z_{\alpha}=V_{\alpha}-V_{0}$ and taking the difference of the above two equations we obtain

$$
\begin{aligned}
\frac{\partial Z_{\alpha}}{\partial t}+\nu A Z_{\alpha}= & -P_{L}\left[\left(\mathcal{R}_{\alpha} V_{\alpha} \cdot \nabla\right) V_{\alpha}\right]+P_{L}\left[\left(V_{0} \cdot \nabla\right) V_{0}\right] \\
& -P_{L}\left[V_{\alpha j} \nabla\left(\left\{R_{\alpha}-I\right\} V_{\alpha}\right)_{j}\right]-f P_{L} J P_{L}\left[\mathcal{R}_{\alpha} V_{\alpha}-V_{0}\right] .
\end{aligned}
$$

Note that

$$
\begin{aligned}
P_{L}\left[\left(\mathcal{R}_{\alpha} V_{\alpha} \cdot \nabla\right) V_{\alpha}\right]-P_{L}\left[\left(V_{0} \cdot \nabla\right) V_{0}\right]= & P_{L}\left[\left(\mathcal{R}_{\alpha} V_{\alpha} \cdot \nabla\right) Z_{\alpha}\right]+P_{L}\left[\left(\mathcal{R}_{\alpha} V_{\alpha} \cdot \nabla\right) V_{0}\right] \\
& +P_{L}\left[\left(Z_{\alpha} \cdot \nabla\right) V_{0}-\left(V_{\alpha} \cdot \nabla\right) V_{0}\right] \\
= & P_{L}\left[\left(\mathcal{R}_{\alpha} V_{\alpha} \cdot \nabla\right) Z_{\alpha}\right]+P_{L}\left[\left(Z_{\alpha} \cdot \nabla\right) V_{0}\right] \\
& +P_{L}\left[\left(\left\{\mathcal{R}_{\alpha}-I\right\} V_{\alpha} \cdot \nabla\right) V_{0}\right] .
\end{aligned}
$$

Then (8.8) becomes

$$
\begin{aligned}
\frac{\partial Z_{\alpha}}{\partial t}+\nu A Z_{\alpha}= & -P_{L}\left[\left(\mathcal{R}_{\alpha} V_{\alpha} \cdot \nabla\right) Z_{\alpha}\right]-P_{L}\left[\left(Z_{\alpha} \cdot \nabla\right) V_{0}\right] \\
& -P_{L}\left[\left(\left\{\mathcal{R}_{\alpha}-I\right\} V_{\alpha} \cdot \nabla\right) V_{0}\right]-P_{L}\left[V_{\alpha j} \nabla\left(\left\{R_{\alpha}-I\right\} V_{\alpha}\right)_{j}\right] \\
& -f P_{L} J P_{L}\left[\mathcal{R}_{\alpha} V_{\alpha}-V_{0}\right] .
\end{aligned}
$$

Taking the inner product (8.9) with $Z_{\alpha}$ gives

$$
\begin{aligned}
\frac{1}{2} \frac{\partial}{\partial t}\left|Z_{\alpha}\right|^{2}+\nu\left\|Z_{\alpha}\right\|^{2}= & -\int\left[\left(Z_{\alpha} \cdot \nabla\right) V_{0}\right] \cdot Z_{\alpha} d x \\
& -\int\left[\left(\left\{\mathcal{R}_{\alpha}-I\right\} V_{\alpha} \cdot \nabla\right) V_{0}\right] \cdot Z_{\alpha} d x \\
& -\int\left[V_{\alpha j} \nabla\left(\left\{R_{\alpha}-I\right\} V_{\alpha}\right)_{j}\right] \cdot Z_{\alpha} d x \\
& -\int\left[f P_{L} J P_{L}\left(\mathcal{R}_{\alpha} V_{\alpha}-V_{0}\right)\right] \cdot Z_{\alpha} d x .
\end{aligned}
$$

Note that $<P_{L}\left[\left(\mathcal{R}_{\alpha} V_{\alpha} \cdot \nabla\right) Z_{\alpha}\right], Z_{\alpha}>=0$. We assess the estimation on the right hand side:

(i)

$$
\begin{aligned}
\left|\int\left[\left(Z_{\alpha} \cdot \nabla\right) V_{0}\right] \cdot Z_{\alpha} d x\right| & \leq\left\|Z_{\alpha}\right\|_{L^{6}}\left|\nabla V_{0}\right|\left\|Z_{\alpha}\right\|_{L^{3}} \\
& \leq c_{1}\left\|Z_{\alpha}\right\|\left\|V_{0}\right\|\left|Z_{\alpha}\right|^{1 / 2}\left\|Z_{\alpha}\right\|^{1 / 2} \\
& =c_{1}\left\|Z_{\alpha}\right\|^{3 / 2}\left\|V_{0}\right\|\left|Z_{\alpha}\right|^{1 / 2} \\
& \leq \frac{\nu}{8}\left\|Z_{\alpha}\right\|^{2}+\frac{54 c_{1}^{2}}{\nu^{3}}\left\|V_{0}\right\|^{4}\left|Z_{\alpha}\right|^{2} .
\end{aligned}
$$

In each of (ii), (iii), and (iv) below, it is Lemma 8.9 that is crucial to obtain third inequalities. 
(ii)

$$
\begin{aligned}
\left|\int\left[\left(\left\{\mathcal{R}_{\alpha}-I\right\} V_{\alpha} \cdot \nabla\right) V_{0}\right] \cdot Z_{\alpha} d x\right| & =\left|\int\left[\left(\left\{\mathcal{R}_{\alpha}-I\right\} V_{\alpha} \cdot \nabla\right) Z_{\alpha}\right] \cdot V_{0} d x\right| \\
& \leq\left\|\left\{\mathcal{R}_{\alpha}-I\right\} V_{\alpha}\right\|_{L^{4}}\left\|Z_{\alpha}\right\|\left\|V_{0}\right\|_{L^{4}} \\
& \leq c_{2}\left|\left\{\mathcal{R}_{\alpha}-I\right\} V_{\alpha}\right|^{1 / 4}\left\|\left\{\mathcal{R}_{\alpha}-I\right\} V_{\alpha}\right\|^{3 / 4} \\
& \quad\left|V_{0}\right|^{1 / 4}\left\|V_{0}\right\|^{3 / 4}\left\|Z_{\alpha}\right\| \\
& \leq c_{2} \epsilon\left\|V_{\alpha}\right\|^{1 / 4}\left\|V_{\alpha}\right\|_{2}^{3 / 4}\left|V_{0}\right|^{1 / 4}\left\|V_{0}\right\|^{3 / 4}\left\|Z_{\alpha}\right\| \\
& \leq c_{2} \epsilon \lambda_{1}^{-1 / 4}\left\|V_{\alpha}\right\|_{2}\left\|V_{0}\right\|\left\|Z_{\alpha}\right\| \\
& \leq \frac{\nu}{4}\left\|Z_{\alpha}\right\|^{2}+\frac{c_{2}^{2} \epsilon^{2}}{\nu \sqrt{\lambda}}\left\|V_{\alpha}\right\|_{2}^{2}\left\|V_{0}\right\|^{2} .
\end{aligned}
$$

(iii)

$$
\begin{aligned}
\left|\int\left[V_{\alpha j} \nabla\left(\left\{R_{\alpha}-I\right\} V_{\alpha}\right)_{j}\right] \cdot Z_{\alpha} d x\right| & =\left|\int\left[\left(Z_{\alpha} \cdot \nabla\right) V_{\alpha}\right] \cdot\left\{R_{\alpha}-I\right\} V_{\alpha} d x\right| \\
& \leq\left\|Z_{\alpha}\right\|_{L^{4}}\left\|V_{\alpha}\right\|\left\|\left(\left\{R_{\alpha}-I\right\} V_{\alpha}\right)\right\|_{L^{4}} \\
& \leq c_{3}\left|\left\{R_{\alpha}-I\right\} V_{\alpha}\right|^{1 / 4}\left\|\left\{R_{\alpha}-I\right\} V_{\alpha}\right\|^{3 / 4} \\
& \left\|V_{\alpha}\right\|\left|Z_{\alpha}\right|^{1 / 4}\left\|Z_{\alpha}\right\|^{3 / 4} \\
& \leq c_{3} \epsilon\left\|V_{\alpha}\right\|^{1 / 4}\left\|V_{\alpha}\right\|_{2}^{3 / 4}\left\|V_{\alpha}\right\|\left|Z_{\alpha}\right|^{1 / 4}\left\|Z_{\alpha}\right\|^{3 / 4} \\
& \leq c_{3} \epsilon \lambda_{1}^{-1 / 4}\left\|V_{\alpha}\right\|_{2}\left\|V_{\alpha}\right\|\left\|Z_{\alpha}\right\| \\
& \leq \frac{\nu}{4}\left\|Z_{\alpha}\right\|^{2}+\frac{c_{3}^{2} \epsilon^{2}}{\nu \sqrt{\lambda_{1}}}\left\|V_{\alpha}\right\|_{2}^{2}\left\|V_{\alpha}\right\|^{2} .
\end{aligned}
$$

(iv)

$$
\begin{aligned}
\left|\int\left[f P_{L} J P_{L}\left(\mathcal{R}_{\alpha} V_{\alpha}-V_{0}\right)\right] \cdot Z_{\alpha} d x\right| \leq & \left|\int\left[f P_{L} J P_{L}\left(\mathcal{R}_{\alpha}-I\right) V_{\alpha}\right] \cdot Z_{\alpha} d x\right| \\
& +\left|\int f P_{L} J P_{L} Z_{\alpha} \cdot Z_{\alpha} d x\right| \\
\leq & \left|f P_{L} J P_{L}\left(\mathcal{R}_{\alpha}-I\right) V_{\alpha}\right|\left|Z_{\alpha}\right| \\
\leq & \epsilon f\left\|V_{\alpha}\right\|\left|Z_{\alpha}\right| \\
\leq & \frac{\nu}{8}\left|Z_{\alpha}\right|^{2}+\frac{2 \epsilon^{2} f^{2}}{\nu}\left\|V_{\alpha}\right\|^{2} .
\end{aligned}
$$

Collecting all the estimates, we get

$$
\begin{aligned}
\frac{\partial}{\partial t}\left|Z_{\alpha}\right|^{2}+\frac{3 \nu}{4}\left\|Z_{\alpha}\right\|^{2} \leq & 2\left(\frac{c^{2}}{\nu^{3}}\left\|V_{0}\right\|^{4}+\frac{\nu}{8}\right)\left|Z_{\alpha}\right|^{2} \\
& +\frac{2 c^{2} \epsilon^{2}}{\nu \sqrt{\lambda_{1}}}\left\|V_{\alpha}\right\|_{2}^{2}\left(\left\|V_{0}\right\|^{2}+\left\|V_{\alpha}\right\|^{2}\right)+\frac{\epsilon^{2} f^{2}}{4},
\end{aligned}
$$

where $c=\max \left\{54 c_{1}, c_{2}, c_{3}, 1\right\}$ and $\epsilon$ arbitrary small number in Lemma 8.9. Write $y=\left|Z_{\alpha}\right|^{2}$. Then, from (8.10)

$$
\frac{d y}{d t} \leq g y+h, \quad \forall t \in[0, T],
$$


where

$$
\begin{aligned}
& y=\left|Z_{\alpha}\right|^{2}=\left|V_{\alpha}-V_{0}\right|^{2} \\
& g=2\left(\frac{c^{2}}{\nu^{3}}\left\|V_{0}\right\|^{4}+\frac{\nu}{8}\right) \leq K_{1}=2\left(\frac{c^{2}}{\nu^{3}}\left(M_{1}^{\prime}\right)^{4}+\frac{\nu}{8}\right) \\
& h=\frac{2 c^{2} \epsilon^{2}}{\nu \sqrt{\lambda_{1}}}\left\|V_{\alpha}\right\|_{2}^{2}\left(\left\|V_{0}\right\|^{2}+\left\|V_{\alpha}\right\|^{2}\right)+\frac{2 \epsilon^{2} f^{2}}{\nu}\left\|V_{\alpha}\right\|^{2} \leq \epsilon^{2} K_{2}+\epsilon^{2} f^{2} K_{3} .
\end{aligned}
$$

Note that $K_{1}, K_{2}=\frac{2 c^{2}}{\nu \sqrt{\lambda_{1}}}\left(M_{2 \beta}^{\prime}\right)^{2}\left(M_{1}^{\prime}\right)^{2}$, and $K_{3}=\frac{2}{\nu}\left(M_{1 \beta}^{\prime}\right)^{2}$, are absolute constants on $[0, T]$.

Choose $\epsilon$ and $\tilde{\epsilon}$ such that

$$
\epsilon^{2} \leq \frac{\hat{\eta}}{2 K_{2}} \text { and } \tilde{\epsilon}^{2} \leq \frac{\hat{\eta}}{2 K_{3}}\left(\frac{1}{f^{2}}\right)^{1+\theta} \quad \text { with } \theta>0
$$

Here $\hat{\eta}$ will be determined later. Let $\epsilon^{*}=\min \{\epsilon, \tilde{\epsilon}\}$ and let $\alpha<\tilde{\alpha}\left(\epsilon^{*}\right)$ to get the small error estimate in Lemma 8.9. Then

$$
h(t) \leq \hat{\eta}, \quad \forall t \in\left[T_{*}, T\right] .
$$

Applying the classical Gronwall's inequality over an interval $\left(t_{1}, t\right) \subset\left[T_{*}, T\right]$ we obtain

$$
\begin{aligned}
y(t) & \leq e^{K_{1}\left(t-t_{1}\right)} y\left(t_{1}\right)+\frac{\hat{\eta}}{K_{1}}\left[e^{K_{1}\left(t-t_{1}\right)}-1\right] \\
& \leq e^{K_{1}\left(t-t_{1}\right)} y\left(t_{1}\right)+\frac{\hat{\eta}}{K_{1}} e^{K_{1}\left(t-t_{1}\right)}, \quad \forall t, t_{1} \text { such that } T_{*} \leq t_{1} \leq t \leq T .
\end{aligned}
$$

Let us assume that the two solutions are identical at some time $t_{1} \geq T_{*}$. Then $y\left(t_{1}\right)=0$, and we get

$$
y(t) \leq \frac{\hat{\eta}}{K_{1}} e^{K_{1}\left(t-t_{1}\right)}, \quad \forall t \in\left[t_{1}, T\right], \forall t_{1} \geq T_{*} .
$$

This implies

$$
\sup _{\tau \in\left[t_{1}, T\right]} y(\tau) \leq \frac{\hat{\eta}}{K_{1}} e^{K_{1}\left(T-T_{*}\right)} .
$$

Choosing $\hat{\eta}$ such that $\left[\hat{\eta} \exp \left(K_{1}\left(T-T_{*}\right)\right)\right] / K_{1}<\eta$ yields

$$
\sup _{\tau \in\left[t_{1}, T\right]} y(\tau) \leq \eta \rightarrow 0 \quad \text { as } \quad \eta \rightarrow 0
$$

A similar lemma can be established for the $H^{1}$-norm distance. Proof is provided in the Appendix, Section C.

LEMMA 8.13. Let $[0, T]$ be a time interval on which the solutions of $3 D R N S E$ and $3 D R N S-\alpha$ equations are in respective absorbing sets in $B_{0,2}$ in $H^{2}$ and $B_{\alpha, \beta}$ in $H^{\beta}, \beta>5 / 2$, and $T$ arbitrary but fixed. Assume $f \geq f^{*}$, where $f^{*}$ is defined in Theorem 6.7. Consider two solutions, $V_{0}$ and $V_{\alpha}$, of the $3 D$ rotating Navier-Stokes equations and the $3 D$ rotating Navier-Stokes- $\alpha$ equations, respectively, with the same 
initial data at some time $t_{1}$. Then two solutions uniformly converge as $\alpha \rightarrow 0$ in $H^{1}$ for all $t \in\left[t_{1}, T\right]$ in the following sense: For any $\eta>0, \exists \tilde{\alpha}\left(\eta, \frac{1}{f}, T, M_{\beta}^{\prime}, M_{2}^{\prime}\right)$ such that, for any $\alpha<\tilde{\alpha}\left(\eta, \frac{1}{f}, T, M_{\beta}^{\prime}, M_{2}^{\prime}\right)$,

$$
\left\|V_{\alpha}(t)-V_{0}(t)\right\|_{1} \leq \eta, \quad \forall t \in\left[t_{1}, T\right], t_{1} \geq T_{*},
$$

and, for fixed $f \geq f^{*}, \eta \rightarrow 0$ as $\tilde{\alpha} \rightarrow 0$. The limit is uniform in $f$ for $f^{*} \leq f \leq f_{\max }$. Moreover, $\tilde{\alpha}\left(\eta, \frac{1}{f}, T, M_{\beta}^{\prime}, M_{2}^{\prime}\right) \rightarrow 0$ as $\eta \rightarrow 0$ and $\frac{1}{f} \rightarrow 0$. (Here, $T_{\alpha 0}=$ the initial time when $V_{\alpha}(t)$ enters into an absorbing set, $T_{0}=$ the initial time when $V_{0}(t)$ enters into an absorbing set, and $T_{*}=\max \left\{T_{\alpha 0}, T_{0}\right\}$ ).

\section{Physical implications: nonlinear scaling laws of parameter $\alpha$}

In conclusion, this paper establishes under what conditions solutions to the geophysical fast rotating Navier-Stokes- $\alpha$ equations uniformly converge to those of the fast rotating classical Navier-Stokes equations as $\alpha \rightarrow 0^{+}$and how respective exponential attractors converge in the Hausdorff semi-distance as $\alpha \rightarrow 0^{+}$. In particular, the proofs of Lemma 8.12 and 8.13 clearly show that $\alpha$ must follow a nonlinear scaling constraint with respect to $1 / f$, the natural fast scale of rotating problem, and the enstrophy in order to achieve uniform convergence:

(i) From (8.6) in the proof of Lemma 8.9, we have

$$
\alpha^{4}<\frac{1}{\check{M}^{4}} \frac{\epsilon / 2}{1-\epsilon / 2} \approx\left(\frac{\epsilon}{2}\right)^{2 / \beta} \frac{\epsilon / 2}{1-\epsilon / 2}, \text { where } \beta>0 \text {. }
$$

(ii) Let $\eta$ be the error in Lemma 8.12 (and Lemma 8.13). From Eq. (8.11), we obtain

$$
\epsilon^{2} \lesssim \frac{\eta^{2}}{2 K_{3}}\left(\frac{1}{f^{2}}\right)
$$

where $K_{3}=\left\|V_{\alpha}\right\|_{1}^{2}$ (enstrophy). Note that we have $\eta^{2}$ instead of $\hat{\eta}$ on the right-hand side.

(iii) When $\beta=1$ in (9.1), the scaling is nonlinear for the relative distance of RNS and RNS- $\alpha$ solution trajectories in the enstrophy norm, and with Constant of dimension $L^{2} / T^{3}$ :

$$
\begin{aligned}
\alpha^{4} & <\text { Constant }\left(\frac{\eta^{2}}{\left\|V_{\alpha}\right\|_{1}^{2} f^{2}}\right)^{3 / 2} \\
& =\text { Constant }\left(\frac{\left\|V_{\alpha}(t)-V_{0}(t)\right\|_{1}}{\left\|V_{\alpha}\right\|_{1}}\right)^{3} \frac{1}{f^{3}}
\end{aligned}
$$

to ensure the $\eta$-errors in Lemma 8.12 (and Lemma 8.13) on $(0, T)$. The constant, of course, depends on $T$.

Future extension of this work will consider the $\alpha$-Primitive equations of geophysical fluid dynamics, that is the Boussinesq equations with rotation (cf., [8]-[11], [13]).

\section{Appendix A. Proof of Theorem 4.1.}

Proof. $L^{2}$-estimate of $\bar{w}$ : Inner product (4.1) with $\bar{w}$ yields

$$
\begin{aligned}
\frac{1}{2} \frac{\partial}{\partial t}|\bar{w}|^{2}+\nu\|\bar{w}\|^{2} & =<\tilde{B}_{I}^{\alpha}(\bar{w}, \bar{w}), \bar{w}>+<\bar{F}, \bar{w}> \\
& \leq\left|<\mathcal{R}_{\alpha} \bar{w} \times \operatorname{curl} \bar{w}, \bar{w}>\right|+\|\bar{F}\|_{H_{1}^{*}}\|\bar{w}\| \\
& \leq \frac{\nu}{4}\|\bar{w}\|+\frac{1}{\nu}\|\bar{F}\|_{H_{1}^{*}}^{2}+|<\bar{u} \times \operatorname{curl} \bar{w}, \bar{w}>|
\end{aligned}
$$


where $\bar{u}=\mathcal{R}_{\alpha} \bar{w}=\left(1-\alpha^{2} \Delta\right)^{-1} \bar{w}$. Then we have

$$
\frac{1}{2} \frac{\partial}{\partial t}|\bar{w}|^{2}+\frac{3 \nu}{4}\|\bar{w}\|^{2} \leq \frac{1}{\nu}\|\bar{F}\|_{H_{1}^{*}}^{2}+|<\bar{u} \times \operatorname{curl} \bar{w}, \bar{w}>|
$$

Using Hölder's and Young's inequality, we get the following estimate on the nonlinear term:

$$
|<\bar{u} \times \operatorname{curl} \bar{w}, \bar{w}>| \leq\left. c|\bar{u}|^{\frac{1}{2}}\left\|\left.\bar{u}\right|^{\frac{1}{2}}|\bar{w}|^{\frac{1}{2}}\right\| \bar{w}\left\|^{\frac{3}{2}} \leq \frac{\nu}{4}\right\| \bar{w}\left\|^{2}+\frac{\tilde{c}}{\nu^{3}}|\bar{u}|^{2}\right\| \bar{u}\right|^{2}|\bar{w}|^{2}
$$

where $\tilde{c}=\frac{27}{4} c^{4}$. Substituting this into (A.1) yields

$$
\frac{\partial}{\partial t}|\bar{w}|^{2}+\left.\nu\left\|\left.\bar{w}\right|^{2} \leq \frac{2}{\nu}\right\| \bar{F}\right|_{H_{1}^{*}}+\frac{\tilde{c}}{\nu^{3}}|\bar{u}|^{2}\|\bar{u}\|^{2}|\bar{w}|^{2} .
$$

Using (2.7) we would get

$$
|\bar{u}|^{2} \leq|\bar{u}|^{2}+\left.\alpha^{2}|| \bar{u}\right|^{2} \leq\left|\bar{u}_{0}\right|^{2}+\alpha^{2}\left\|\bar{u}_{o}\right\|^{2}+\frac{M_{0 F}^{2}}{\nu^{2} \lambda_{1}} \equiv K_{1} .
$$

Note that $K_{1}$ is an absolute constant. Then, Eq. (A) yields

$$
\frac{\partial}{\partial t}|\bar{w}|^{2}+\nu\|\bar{w}\|^{2} \leq \frac{2}{\nu}\|\bar{F}\|_{H_{1}^{*}}^{2}+\frac{\tilde{c} K_{1}}{\nu^{3}}\|\bar{u}\|^{2}|\bar{w}|^{2}
$$

Let $y=|\bar{w}|^{2}$. Then Eq. (A.2) becomes

$$
\frac{d y}{d t} \leq g y+h, \quad \forall t \in[0, T],
$$

where $g=\frac{\tilde{c} K_{1}}{\nu^{3}}\|\bar{u}\|^{2}$ and $h=\frac{2}{\nu}\|\bar{F}\|_{H_{1}^{*}}^{2}$. The Gronwall's inequality gives

$$
y(t) \leq\left|\bar{w}_{0}\right|^{2} \exp \left[\frac{\tilde{c} K_{1} M_{0 F}^{2}}{\nu^{5}} T\right]+\frac{2 \nu^{4}}{\tilde{c} K_{1}}\left(1-\exp \left[\frac{\tilde{c} K_{1} M_{0 F}^{2}}{\nu^{5}} T\right]\right) \equiv \bar{\rho}_{H}^{2}(T), \forall \tilde{t} \in[0, T],
$$

and hence

$$
\bar{w} \in L^{\infty}(0, T ; H), \quad \forall T>0 .
$$

Furthermore, from (2.6), (4.5), (A.2), and (A.4), we obtain the time averaging of $\bar{w}$ in $H^{1}$ :

$$
\nu \int_{0}^{T}\|\bar{w}\|^{2} \leq\left[\frac{2 M_{0 F}^{2}}{\nu}+\frac{\tilde{c} K_{1} M_{0 F}^{2}}{\nu^{5}}|\bar{w}|_{L^{\infty}(0, T ; H)}\right] T \equiv K_{2}(T) .
$$

This implies

$$
\bar{w} \in L^{2}\left(0, T ; H^{1}\right), \quad \forall T>0 .
$$

$H^{1}$-estimate of $\bar{w}$ : By taking inner product (4.1) with $A \bar{w}$ we get

$$
\frac{1}{2} \frac{d}{d t}|| \bar{w} \|^{2}+\frac{3 \nu}{4}|A \bar{w}|^{2} \leq \frac{|\bar{F}|^{2}}{\nu}+|<\bar{u} \times \bar{w}, A \bar{w}>| .
$$


Note that $|<\bar{u} \times \bar{w}, A \bar{w}>|=|<\operatorname{curl}(\bar{u} \times \bar{w}), \operatorname{curl} \bar{w}>|$. Using the identity $\operatorname{curl}(A \times$ $B)=(B \cdot \nabla) A-(A \cdot \nabla) B$ we can estimate the nonlinear term:

$|<\operatorname{curl}(\bar{u} \times \bar{w}), \operatorname{curl} \bar{w}\rangle|\leq|\langle(\operatorname{curl} \bar{w} \cdot \nabla) \bar{u}, \operatorname{curl} \bar{w}\rangle|+|\langle(\bar{u} \cdot \nabla) \operatorname{curl} \bar{w}, \operatorname{curl} \bar{w}\rangle \mid$

(the second term is 0$) \rightarrow=|<(\operatorname{curl} \bar{w} \cdot \nabla) \bar{u}, \operatorname{curl} \bar{w}>|$

$$
\begin{aligned}
& \leq\|\operatorname{curl} \bar{w}\|_{L^{4}}^{2}|\nabla \bar{u}| \\
& \leq c|\operatorname{curl} \bar{w}|\|\operatorname{curl} \bar{w}\|\|\bar{u}\| \\
& \leq c|| \bar{w}\||A \bar{w}|\| \bar{u} \| \\
& \leq \frac{\nu}{4}|A \bar{w}|^{2}+\frac{c^{2}}{\nu}\|\bar{u}\|^{2}\|\bar{w}\|^{2} .
\end{aligned}
$$

This gives

$$
\frac{d}{d t}\|\bar{w}\|^{2}+\nu|A \bar{w}|^{2} \leq \frac{2}{\nu}|\bar{F}|^{2}+\frac{2 c^{2}}{\nu}\|\bar{u}\|^{2}\|\bar{w}\|^{2} .
$$

Let $y=\|\bar{w}\|^{2}$. Then, from (A.8), we obtain

$$
\frac{d y}{d t} \leq g y+h, \quad \forall t \in[0, T]
$$

where $g=\frac{2 c^{2}}{\nu}\|\bar{u}\|^{2}$ and $h=\frac{2}{\nu}|\bar{F}|^{2}$. Following the usual line of arguments with the Gronwall's inequality provides

$$
\bar{w} \in L^{\infty}\left(0, T ; H^{1}\right) \bigcap L^{2}\left(0, T ; H^{2}\right), \quad \forall T>0 .
$$

Now, thanks to (A.4), (A.6), and (A.9), the existence and uniqueness of regular solutions of barotropic limit equations follow in a standard way like for the classical 2D NSE (cf. [18], [29], [33], [35], [36]).

\section{Appendix B. Proof of theorem 4.2 .}

Recall that $\tilde{B}_{I I}^{\alpha}\left(\bar{w}, w^{\perp}\right)=B_{1}\left(\bar{w}, w^{\perp}\right)+B_{2}\left(\bar{w}, w^{\perp}\right)+B_{3}\left(w^{\perp}, \bar{w}\right)+B_{4}\left(w^{\perp}, \bar{w}\right) . \quad$ In Fourier space, the bilinear operator $\tilde{B}_{I I}^{\alpha}\left(\bar{w}, w^{\perp}\right)$ is expressed as following; for any wavenumber $n \in \mathbb{Z}^{3}$,

$$
\begin{aligned}
& B_{1}\left(\bar{w}, w^{\perp}\right)_{n}=-i \sum_{K_{24}} \frac{1}{1+\alpha^{2}|\check{k}|^{2}} P_{n}\left[\left(\check{m} \cdot \bar{w}_{k}\right) w_{m}^{\perp}\right] \\
& B_{2}\left(\bar{w}, w^{\perp}\right)_{n}=\frac{i}{2} \sum_{K_{24}} \frac{1}{1+\alpha^{2}|\check{k}|^{2}} P_{n}\left[\left(\bar{w}_{k} \cdot w_{m}^{\perp}\right) \check{m}+\left(\check{k} \cdot w_{m}^{\perp}\right) \bar{w}_{k}\right], \\
& B_{3}\left(w^{\perp}, \bar{w}\right)_{n}=\frac{i}{2} \sum_{K_{34}} \frac{1}{1+\alpha^{2}|\check{n}|^{2}} P_{n}\left[\left(w_{k}^{\perp} \cdot \bar{w}_{m}\right) \check{m}-\left(\check{m} \cdot w_{k}^{\perp}\right) \bar{w}_{m}\right], \\
& B_{4}\left(w^{\perp}, \bar{w}\right)_{n}=-\frac{i}{2} \sum_{K_{34}} \frac{1}{1+\alpha^{2}|\check{n}|^{2}} \frac{1}{|\check{n}|^{2}} P_{n}\left[\check{n} \times\left\{\left(\check{k} \times w_{k}^{\perp}\right) \times\left(\check{m} \times \bar{w}_{m}\right)\right\}\right] .
\end{aligned}
$$

Recall that

$$
K_{24}=\left\{(k, m, n) \in \mathbb{Z}^{9}\left|k_{3}=0, m_{3}=n_{3} \neq 0,\right| \check{m}|=| \check{n} \mid, k+m=n\right\},
$$

and

$$
K_{34}=\left\{(k, m, n) \in \mathbb{Z}^{9}\left|m_{3}=0, k_{3}=n_{3} \neq 0,\right| \check{k}|=| \check{n} \mid, k+m=n\right\} .
$$


For the regularity of catalytic limit $\alpha$-equations we use techniques developed in [6]. Let $r \geq 0$ and $s_{3} \in \mathbb{Z}$. We introduce the operator $\pi_{r, s_{3}}$ restriction of functions on $\mathbb{Z}^{3}$ with fixed $n_{3}$ to the points of the lattice on the ellipse $\left|\check{n}_{h}\right|^{2}=n_{1}^{2}+n_{2}^{2} / a_{2}^{2}=r^{2}$ defined as a projection, for each $n=\left(n_{h}, n_{3}\right) \in \mathbf{Z}^{3}$,

$$
\left(\pi_{r, s_{3}} w^{\perp}\right)_{\left(n_{h}, n_{3}\right)}= \begin{cases}w_{\left(n_{h}, n_{3}\right)}^{\perp} & \text { if }\left|n_{h}\right|^{2}=r^{2} \text { and } n_{3}=s_{3} \\ 0 & \text { otherwise }\end{cases}
$$

Recall that $\left|n_{h}\right|^{2}=n_{1}^{2}+n_{2}^{2} / a_{2}^{2}$. This projection with fixed $r$ and $s_{3}$ is finitedimensional. Denote $w_{\left(n_{h}, n_{3}\right)}^{r}=\left(\pi_{r, s_{3}} w^{\perp}\right)_{\left(n_{h}, n_{3}\right)}$ and let $r=r_{j}$ be the increasing sequence of numbers defined by $n_{1}^{2}+n_{2}^{2} / a_{2}^{2}=r^{2},\left(n_{1}, n_{2}\right) \in \mathbb{Z}^{2}$. Then we have the orthogonal decomposition

$$
w^{\perp}=\sum_{j, s_{3}} \pi_{r_{j}, s_{3}} w^{\perp}
$$

Since the sums in the bilinear operators $B_{i}\left(\bar{w}, w^{\perp}\right)$ are over $\left|\check{m}_{h}\right|^{2}=\left|\check{n}_{h}\right|^{2}, m_{3}=n_{3}, k_{3}=$ 0 on $K_{24}$ and $\left|\check{k}_{h}\right|^{2}=\left|\check{n}_{h}\right|^{2}, k_{3}=n_{3}, m_{3}=0$ on $K_{34}$, the functions $w_{\left(n_{h}, n_{3}\right)}^{r}$ are coupled with both of $w_{\left(m_{h}, n_{3}\right)}^{r}$ (on $\left.K_{24}\right)$ and $w_{\left(k_{h}, n_{3}\right)}^{r}$ (on $\left.K_{34}\right)$. In addition, $K_{24} \cap K_{34}=$ $\phi$. Therefore, the catalytic limit $\alpha$-equations decouple into a countable family of subsystems (each of subsystem is decoupled with respect to $n_{3}$.):

$$
\begin{aligned}
& \partial_{t} w_{\left(n_{h}, n_{3}\right)}^{r}=-\nu|\check{n}|^{2} w_{\left(n_{h}, n_{3}\right)}^{r} \\
& -i \sum_{\substack{m_{3}=n_{3} \\
\left|m_{h}\right|=\left|n_{h}\right|=r \\
k_{h}+m_{h}=n_{h}}} \frac{1}{1+\alpha^{2}|\check{k}|^{2}} P_{n}\left[\left(\check{m} \cdot \bar{w}_{k}\right) w_{\left(m_{h}, n_{3}\right)}^{r}\right] \\
& +\frac{i}{2} \sum_{\substack{m_{3}=n_{3} \\
\left|m_{h}\right|=\left|n_{h}\right|=r \\
k_{h}+m_{h}=n_{h}}} \frac{1}{1+\alpha^{2}|\check{k}|^{2}} P_{n}\left[\left(\bar{w}_{k} \cdot w_{\left(m_{h}, n_{3}\right)}^{r}\right) \check{m}+\left(\check{k} \cdot w_{\left(m_{h}, n_{3}\right)}^{r}\right) \bar{w}_{k}\right] \\
& +\frac{i}{2} \sum_{\substack{k_{3}=n_{3} \\
\left|k_{h}\right|=\left|n_{h}\right|=r \\
k_{h}+m_{h}=n_{h}}} \frac{1}{1+\alpha^{2}|\check{n}|^{2}} P_{n}\left[\left(w_{\left(k_{h}, n_{3}\right)}^{r} \cdot \bar{w}_{m}\right) \check{m}-\left(\check{m} \cdot w_{\left(k_{h}, n_{3}\right)}^{r}\right) \bar{w}_{m}\right] \\
& -\frac{i}{2} \sum_{\substack{k_{3}=n_{3} \\
\left|k_{h}\right|=\left|n_{h}\right|=r \\
k_{h}+m_{h}=n_{h}}} \frac{1}{1+\alpha^{2}|\check{n}|^{2}} \frac{1}{|\check{n}|^{2}} P_{n}\left[\check{n} \times\left\{\left(\check{k} \times w_{\left(k_{h}, n_{3}\right)}^{r}\right) \times\left(\check{m} \times \bar{w}_{m}\right)\right\}\right] \\
& +\bar{F}_{\left(n_{h}, n_{3}\right)}^{\perp} \text {. }
\end{aligned}
$$

Note that every sum contains all solutions of Diophantine equations: $k_{1}^{2}+k_{2}^{2} / a_{2}^{2}=n^{2}=$ $n_{1}^{2}+n_{2}^{2} / a_{2}^{2}$. Generically, there are 4 solutions corresponding to $k_{1}= \pm n_{1}, k_{2}= \pm n_{2}$, except for a rare set of special rational $a_{2}^{2}, a_{3}^{2}$, where there can be a larger, but finite, set of integer solutions. From the proof of existence and regularity of solutions of 2D3C NS$\alpha$ equations, the Fourier coefficients $\bar{w}_{k}(t)=\bar{w}_{\left(k_{h}, 0\right)}(t)$ and $\bar{v}_{m}(t)=\bar{w}_{\left(m_{h}, 0\right)}(t)$ in the catalytic subsystem are bounded uniformly in $t \in[0, T] ; \bar{w}_{k, h}(t), \bar{v}_{m, h}(t) \in L^{\infty}(0, T ; H)$. We implicitly assume $n_{3} \neq 0$ because $n_{3}=0$ corresponds to the barotropic case. We 
denote, for a fixed $n_{3}$,

$$
\begin{aligned}
& B_{1}^{r, n_{3}}\left(\bar{w}, w^{\perp}\right)_{n}=-i \sum_{\substack{m_{3}=n_{3}=\\
\left|m_{h}\right|=\left|n_{h}\right|=r \\
k_{h}+m_{h}=n_{h}}} \frac{1}{1+\alpha^{2}|\check{k}|^{2}} P_{n}\left[\left(\check{m} \cdot \bar{w}_{k}\right) w_{m}^{\perp}\right] \\
& =-i \sum_{\substack{m_{3}=n_{3} \\
\left|m_{h}\right|=\left|n_{h}\right|=r \\
k_{h}+m_{h}=n_{h}}} \frac{1}{1+\alpha^{2}|\check{k}|^{2}} P_{n}\left[\left(\check{m} \cdot \bar{w}_{k}\right) w_{\left(m_{h}, n_{3}\right)}^{r}\right] \\
& B_{2}^{r, n_{3}}\left(\bar{w}, w^{\perp}\right)_{n}=\frac{i}{2} \sum_{\substack{m_{3}=n_{3}=\\
\left|m_{h}\right|=\left|n_{h}\right|=r \\
k_{h}+m_{h}=n_{h}}} \frac{1}{1+\alpha^{2}|\check{k}|^{2}} P_{n}\left[\left(\bar{w}_{k} \cdot w_{m}^{\perp}\right) \check{m}+\left(\check{k} \cdot w_{m}^{\perp}\right) \bar{w}_{k}\right] \\
& =\frac{i}{2} \sum_{\substack{m_{3}=n_{3} \\
\left|m_{h}\right|=\left|n_{h}\right|=r \\
k_{h}+m_{h}=n_{h}}} \frac{1}{1+\alpha^{2}|\check{k}|^{2}} P_{n}\left[\left(\bar{w}_{k} \cdot w_{\left(m_{h}, n_{3}\right)}^{r}\right) \check{m}+\left(\check{k} \cdot w_{\left(m_{h}, n_{3}\right)}^{r}\right) \bar{w}_{k}\right] \\
& B_{3}^{r, n_{3}}\left(w^{\perp}, \bar{w}\right)_{n}=\frac{i}{2} \sum_{\substack{k_{3}=n_{3} \\
\left|k_{h}\right|=n_{h} \mid=r \\
k_{h}+m_{h}=n_{h}}} \frac{1}{1+\alpha^{2}|\check{n}|^{2}} P_{n}\left[\left(w_{m}^{\perp} \cdot \bar{w}_{m}\right) \check{m}-\left(\check{m} \cdot w_{k}^{\perp}\right) \bar{w}_{m}\right] \\
& =\frac{i}{2} \sum_{\substack{k_{3}=n_{3}=1 \\
\left|k_{h}=\right| n_{h} \mid=r \\
k_{h}+m_{h}=n_{h}}} \frac{1}{1+\alpha^{2}|\check{n}|^{2}} P_{n}\left[\left(w_{\left(k_{h}, n_{3}\right)}^{r} \cdot \bar{w}_{m}\right) \check{m}-\left(\check{m} \cdot w_{\left(k_{h}, n_{3}\right)}^{r}\right) \bar{w}_{m}\right] \\
& B_{4}^{r, n_{3}}\left(w^{\perp}, \bar{w}\right)_{n}=-\frac{i}{2} \sum_{\substack{k_{3}=n_{3} \\
\left|k_{h}\right|=\left|n_{h}\right|=r \\
k_{h}+m_{h}=n_{h}}} \frac{1}{1+\alpha^{2}|\check{n}|^{2}} \frac{1}{|\check{n}|^{2}} P_{n}\left[\check{n} \times\left\{\left(\check{k} \times w_{k}^{\perp}\right) \times\left(\check{m} \times \bar{w}_{m}\right)\right\}\right] \\
& =-\frac{i}{2} \sum_{\substack{k_{3}=n_{3} \\
\left|k_{h}\right|=\left|n_{h}\right|=r \\
k_{h}+m_{h}=n_{h}}} \frac{1}{1+\alpha^{2}|\check{n}|^{2}} \frac{1}{|\check{n}|^{2}} P_{n}\left[\check{n} \times\left\{\left(\check{k} \times w_{\left(k_{h}, n_{3}\right)}^{r}\right) \times\left(\check{m} \times \bar{w}_{m}\right)\right\}\right] .
\end{aligned}
$$

Now we introduce the following operators:

$$
\begin{aligned}
& B_{1}^{r, s_{3}}\left(\bar{w}, w^{\perp}\right)=\sum_{\substack{n_{3}=s_{3} \\
\left|n_{h}\right|=r}} B_{1}^{r, n_{3}}\left(\bar{w}, w^{\perp}\right)_{n} e^{i \check{n} \cdot x} \\
& B_{2}^{r, s_{3}}\left(\bar{w}, w^{\perp}\right)=\sum_{\substack{n_{3}=s_{3} \\
\left|n_{h}\right|=r}} B_{2}^{r, n_{3}}\left(\bar{w}, w^{\perp}\right)_{n} e^{i \check{n} \cdot x} \\
& B_{3}^{r, s_{3}}\left(w^{\perp}, \bar{w}\right)=\sum_{\substack{n_{3}=s_{3} \\
\left|n_{h}\right|=r}} B_{3}^{r, n_{3}}\left(w^{\perp}, \bar{w}\right)_{n} e^{i \check{n} \cdot x} \\
& B_{4}^{r, s_{3}}\left(w^{\perp}, \bar{w}\right)=\sum_{\substack{n_{3}=s_{3} \\
\left|n_{h}\right|=r}} B_{4}^{r, n_{3}}\left(w^{\perp}, \bar{w}\right)_{n} e^{i \check{n} \cdot x} .
\end{aligned}
$$

The operators $B_{j}^{r, s_{3}}(j=1,2,3,4)$ are linear in $w^{\perp}$ for any $\bar{w} \in H$ and they, by construction, act from $H^{s+1}$ into $\pi_{r, s_{3}} H^{s}$.

LEMmA B.1. The four operators $B_{j}^{r, s_{3}}$ for fixed $r$ and $s_{3}$ satisfy the following properties.

1. (a) $\left\|B_{1}^{r, s_{3}}\left(\bar{w}, w^{\perp}\right)\right\|_{s} \leq 2\|\bar{w}\|_{H_{\infty}^{0}}\left\|\pi_{r, s_{3}} w^{\perp}\right\|_{s+1} \leq 2\|\bar{w}\|_{H_{\infty}^{0}}\left\|w^{\perp}\right\|_{s+1}$

(b) $\left\|B_{2}^{r, s_{3}}\left(\bar{w}, w^{\perp}\right)\right\|_{s} \leq(1+2 \sqrt{2})\|\bar{w}\|_{H_{\infty}^{0}}\left\|\pi_{r, s_{3}} w^{\perp}\right\|_{s+1}$

$$
\leq(1+2 \sqrt{2})\|\bar{w}\|_{H_{\infty}^{0}}\left\|w^{\perp}\right\|_{s+1}
$$


(c) $\left\|B_{3}^{r, s_{3}}\left(w^{\perp}, \bar{w}\right)\right\|_{s} \leq\left. 4 \sqrt{2}|| \bar{w}\right|_{H_{\infty}^{0}}\left\|\pi_{r, s_{3}} w^{\perp}\right\|_{s+1} \leq 4 \sqrt{2}|| \bar{w}\left\|_{H_{\infty}^{0}}\right\| w^{\perp} \|_{s+1}$

(d) $\left\|B_{4}^{r, s_{3}}\left(w^{\perp}, \bar{w}\right)\right\|_{s} \leq 2 \sqrt{2}|| \bar{w}\left\|_{H_{\infty}^{0}}\right\| \pi_{r, s_{3}} w^{\perp}\left\|_{s+1} \leq 2 \sqrt{2}\right\| \bar{w}\left\|_{H_{\infty}^{0}}\right\| w^{\perp} \|_{s+1}$.

2. The operators commute with $A^{s}$ and $\partial_{3}^{l}$ for all $s, l$ :

$$
\begin{aligned}
& A^{s} \partial_{3}^{l} B_{1}^{r, s_{3}}\left(\bar{w}, w^{\perp}\right)=B_{1}^{r, s_{3}}\left(\bar{w}, A^{s} \partial_{3}^{l} w^{\perp}\right), \\
& A^{s} \partial_{3}^{l} B_{2}^{r, s_{3}}\left(\bar{w}, w^{\perp}\right)=B_{2}^{r, s_{3}}\left(\bar{w}, A^{s} \partial_{3}^{l} w^{\perp}\right), \\
& A^{s} \partial_{3}^{l} B_{3}^{r, s_{3}}\left(w^{\perp}, \bar{w}\right)=B_{3}^{r, s_{3}}\left(A^{s} \partial_{3}^{l} w^{\perp}, \bar{w}\right), \\
& A^{s} \partial_{3}^{l} B_{4}^{r, s_{3}}\left(w^{\perp}, \bar{w}\right)=B_{4}^{r, s_{3}}\left(A^{s} \partial_{3}^{l} w^{\perp}, \bar{w}\right) .
\end{aligned}
$$

3. The operators are skew-symmetric in $w^{\perp}$ :

$$
\begin{aligned}
& <B_{1}^{r, s_{3}}\left(\bar{w}, w^{\perp}\right), w^{\perp}>=0, \quad<B_{2}^{r, s_{3}}\left(\bar{w}, w^{\perp}\right), w^{\perp}>=0, \\
& <B_{3}^{r, s_{3}}\left(w^{\perp}, \bar{w}\right), w^{\perp}>=0, \quad<B_{4}^{r, s_{3}}\left(w^{\perp}, \bar{w}\right), w^{\perp}>=0 .
\end{aligned}
$$

4. The operator $\operatorname{curl}\left(B_{1}^{r, s_{3}}+B_{2}^{r, s_{3}}+B_{3}^{r, s_{3}}+B_{4}^{r, s_{3}}\right)$ is skew-symmetric in $w^{\perp}$ :

$$
<\operatorname{curl}\left(B_{1}^{r, s_{3}}+B_{2}^{r, s_{3}}+B_{3}^{r, s_{3}}+B_{4}^{r, s_{3}}\right)\left(\bar{w}, w^{\perp}\right), w^{\perp}>=0 .
$$

The catalytic bilinear operator $\tilde{B}_{I I}^{\alpha}\left(\bar{w}, w^{\perp}\right)$ can be expressed as

$$
\begin{aligned}
& B_{1}\left(\bar{w}, w^{\perp}\right)=\sum_{\substack{r \in \mathbb{R}^{+} \\
s_{3} \in \mathbb{Z}}} B_{1}^{r, s_{3}}\left(\bar{w}, w^{\perp}\right) \\
& B_{2}\left(\bar{w}, w^{\perp}\right)=\sum_{\substack{r \in \mathbb{R}^{+} \\
s_{3} \in \mathbb{Z}}} B_{2}^{r, s_{3}}\left(\bar{w}, w^{\perp}\right) \\
& B_{3}\left(w^{\perp}, \bar{w}\right)=\sum_{\substack{r \in \mathbb{R}^{+} \\
s_{3} \in \mathbb{Z}}} B_{3}^{r, s_{3}}\left(w^{\perp}, \bar{w}\right) \\
& B_{4}\left(w^{\perp}, \bar{w}\right)=\sum_{\substack{r \in \mathbb{R}^{+} \\
s_{3} \in \mathbb{Z}^{+}}} B_{4}^{r, s_{3}}\left(w^{\perp}, \bar{w}\right) .
\end{aligned}
$$

\section{Proof of Theorem 4.2:}

Proof. Note that the action of $A^{s}$ in Fourier representation is multiplication by $(-1)^{s}|\breve{n}|^{2 s}$ which is a constant in all of the catalytic subsystem. Analogously $\partial_{3}^{l}$ corresponds to multiplication by a constant $\left(i \check{n}_{3}\right)^{l}$. Then the properties can be obtained from the corresponding assertion of Lemma B.1 using summation in $r$ and $n_{3}$. Here we prove property 2 and 4 only.

Proof of 2: By the definition

$$
\begin{aligned}
<\tilde{B}_{I I}^{\alpha}\left(\bar{w}, w^{\perp}\right), A^{s} w^{\perp}>= & <B_{1}\left(\bar{w}, w^{\perp}\right), A^{s} w^{\perp}>+<B_{2}\left(\bar{w}, w^{\perp}\right), A^{s} w^{\perp}> \\
& +<B_{3}\left(w^{\perp}, \bar{w}\right), A^{s} w^{\perp}>+<B_{4}\left(w^{\perp}, \bar{w}\right), A^{s} w^{\perp}>
\end{aligned}
$$

consider $<B_{1}\left(\bar{w}, w^{\perp}\right), A^{s} w^{\perp}>$.

$$
\begin{aligned}
<B_{1}\left(\bar{w}, w^{\perp}\right), A^{s} w^{\perp}> & =\sum_{\substack{r \in \mathbb{R}^{+} \\
s_{3} \in \mathbb{Z}}}<B_{1}^{r, s_{3}}\left(\bar{w}, w^{\perp}\right), A^{s} w^{\perp}> \\
& =\sum_{\substack{r \in \mathbb{R}^{+} \\
s_{3} \in \mathbb{Z}}}<A^{s} B_{1}^{r, s_{3}}\left(\bar{w}, w^{\perp}\right), w^{\perp}>.
\end{aligned}
$$


Notice that

$$
\begin{aligned}
A^{s} B_{1}^{r, s_{3}}\left(\bar{w}, w^{\perp}\right) & =\sum_{\substack{n_{3}=s_{3} \\
\left|\check{n}_{h}\right|=r}} B_{1}^{r, n_{3}}\left(\bar{w}, w^{\perp}\right)_{n} A^{s} e^{i \check{n} \cdot x} \\
& =\sum_{\substack{n_{3}=s_{3} \\
\left|\check{n}_{h}\right|=r}}(-1)^{s}|\check{n}|^{2 s} B_{1}^{r, n_{3}}\left(\bar{w}, w^{\perp}\right)_{n} e^{i \check{n} \cdot x}
\end{aligned}
$$

Since $n_{3}$ and $\left|\check{n}_{h}\right|=r$ are fixed and $|\check{n}|^{2}=\left|\check{n}_{h}\right|^{2}+\check{n}_{3}^{2},(-1)^{s}|\check{n}|^{2 s}$ can be taken outside the summation. Then,

$$
\begin{aligned}
A^{s} B_{1}^{r, s_{3}}\left(\bar{w}, w^{\perp}\right) & =(-1)^{s}|\check{n}|^{2 s} \sum_{\substack{n_{3}=s_{3} \\
\left|\check{n}_{h}\right|=r}} B_{1}^{r, n_{3}}\left(\bar{w}, w^{\perp}\right)_{n} e^{i \check{n} \cdot x} \\
& =(-1)^{s}|\check{n}|^{2 s} B_{1}^{r, s_{3}}\left(\bar{w}, w^{\perp}\right) .
\end{aligned}
$$

Hence, by property 3 of Lemma B.1,

$$
<B_{1}\left(\bar{w}, w^{\perp}\right), A^{s} w^{\perp}>=\sum_{\substack{r \in \mathbb{R}^{+} \\ s_{3} \in \mathbb{Z}}}(-1)^{s}|\check{n}|^{2 s}<B_{1}^{r, s_{3}}\left(\bar{w}, w^{\perp}\right), w^{\perp}>=0 .
$$

Likewise, we can prove that

$$
<B_{2}\left(\bar{w}, w^{\perp}\right), A^{s} w^{\perp}>=<B_{3}\left(w^{\perp}, \bar{w}\right), A^{s} w^{\perp}>=<B_{4}\left(w^{\perp}, \bar{w}\right), A^{s} w^{\perp}>=0 .
$$

The property 2 follows.

Proof of 4: The method is similar to the above. We consider

$$
\begin{aligned}
<\tilde{B}_{I I}^{\alpha}\left(\bar{w}, w^{\perp}\right), \partial_{3}^{l} w^{\perp}> & =<B_{1}\left(\bar{w}, w^{\perp}\right), \partial_{3}^{l} w^{\perp}>+<B_{2}\left(\bar{w}, w^{\perp}\right), \partial_{3}^{l} w^{\perp}> \\
& +<B_{3}\left(w^{\perp}, \bar{w}\right), \partial_{3}^{l} w^{\perp}>+<B_{4}\left(w^{\perp}, \bar{w}\right), \partial_{3}^{l} w^{\perp}>.
\end{aligned}
$$

Again, it is sufficient to consider only $<B_{1}\left(\bar{w}, w^{\perp}\right), \partial_{3}^{l} w^{\perp}>$. Others follow by a similar procedure.

$$
<B_{1}\left(\bar{w}, w^{\perp}\right), \partial_{3}^{l} w^{\perp}>=\sum_{\substack{r \in \mathbb{R}^{+} \\ s_{3} \in \mathbb{Z}}}<B_{1}^{r, s_{3}}\left(\bar{w}, w^{\perp}\right), \partial_{3}^{l} w^{\perp}>
$$

Here,

$$
\begin{aligned}
<B_{1}^{r, s_{3}}\left(\bar{w}, w^{\perp}\right), \partial_{3}^{l} w^{\perp}> & =\sum_{\substack{n_{3}=s_{3} \\
\left|\check{n}_{h}\right|=r}} B_{1}^{r, n_{3}}\left(\bar{w}, w^{\perp}\right)_{n} \cdot\left(i \check{n}_{3}\right)^{l} w_{-n}^{\perp} \\
& =\left(i \check{n}_{3}\right)^{l} \sum_{\substack{n_{3}=s_{3} \\
\left|\check{n}_{h}\right|=r}} B_{1}^{r, n_{3}}\left(\bar{w}, w^{\perp}\right)_{n} \cdot w_{-n}^{\perp} \\
& =\left(i \check{n}_{3}\right)^{l}<B_{1}^{r, s_{3}}\left(\bar{w}, w^{\perp}\right), w^{\perp}> \\
& =0 \text { by property } 3 \text { of Lemma B.1. }
\end{aligned}
$$

Therefore, the property 4 follows. 


\section{Appendix C. Proof of lemma 8.13.}

Proof. Let $B_{0,2}$ be an absorbing set for $V_{0}$ in $H^{2}$ and let $B_{\alpha, \beta}, \beta>5 / 2$ be an absorbing set for $V_{\alpha}$ in $H^{\beta}$. We assume that $V_{0}$ is in $B_{0,2}$ and $V_{\alpha}$ is in $B_{\alpha, \beta}$ with:

$$
\left\|V_{0}\right\|_{2} \leq M_{2}^{\prime},\left\|V_{\alpha}\right\|_{\beta} \leq M_{\beta}^{\prime},
$$

where $M_{\beta}^{\prime}=\rho_{\beta}$. Let $T$ be an arbitrary, but fixed time.

Take inner product Eq. (8.9) with $A Z_{\alpha}$. Then we get

$$
\begin{aligned}
\frac{1}{2} \frac{\partial}{\partial t}\left\|Z_{\alpha}\right\|^{2}+\nu\left|A Z_{\alpha}\right|^{2}= & -\int\left[\left(\mathcal{R}_{\alpha} V_{\alpha} \cdot \nabla\right) Z_{\alpha}\right] \cdot A Z_{\alpha} d x \\
& -\int\left[\left(Z_{\alpha} \cdot \nabla\right) V_{0}\right] \cdot A Z_{\alpha} d x \\
& -\int\left[\left(\left\{\mathcal{R}_{\alpha}-I\right\} V_{\alpha} \cdot \nabla\right) V_{0}\right] \cdot A Z_{\alpha} d x \\
& -\int\left[V_{\alpha j} \nabla\left(\left\{R_{\alpha}-I\right\} V_{\alpha}\right)_{j}\right] \cdot A Z_{\alpha} d x \\
& -\int\left[f P_{L} J P_{L}\left(\mathcal{R}_{\alpha} V_{\alpha}-V_{0}\right)\right] \cdot A Z_{\alpha} d x .
\end{aligned}
$$

Again, we estimate the nonlinear terms on the right hand side.

(i)

$$
\begin{aligned}
\left|\int\left[\left(\mathcal{R}_{\alpha} V_{\alpha} \cdot \nabla\right) Z_{\alpha}\right] \cdot A Z_{\alpha} d x\right| & \leq\left\|\mathcal{R}_{\alpha} V_{\alpha}\right\|_{L^{6}}\left\|\nabla Z_{\alpha}\right\|_{L^{3}}\left|A Z_{\alpha}\right| \\
& \leq c_{1}\left\|\mathcal{R}_{\alpha} V_{\alpha}\right\|\left\|Z_{\alpha}\right\|^{\frac{1}{2}}\left\|Z_{\alpha}\right\|_{2}^{\frac{1}{2}}\left|A Z_{\alpha}\right| \\
& \leq c_{1}\left\|V_{\alpha}\right\|\left\|Z_{\alpha}\right\|^{\frac{1}{2}}\left|A Z_{\alpha}\right|^{\frac{3}{2}} \\
& \leq \frac{\nu}{8}\left|A Z_{\alpha}\right|^{2}+\frac{54 c_{1}^{4}}{\nu^{3}}\left\|V_{\alpha}\right\|^{4}\left\|Z_{\alpha}\right\|^{2}
\end{aligned}
$$

(ii)

$$
\begin{aligned}
\left|\int\left[\left(Z_{\alpha} \cdot \nabla\right) V_{0}\right] \cdot A Z_{\alpha} d x\right| & \leq\left\|Z_{\alpha}\right\|_{L^{6}}\left\|\nabla V_{0}\right\|_{L^{3}}\left|A Z_{\alpha}\right| \\
& \leq c_{2}\left\|Z_{\alpha}\right\|\left\|V_{0}\right\|^{\frac{1}{2}}\left\|V_{0}\right\|_{2}^{\frac{1}{2}}\left|A Z_{\alpha}\right| \\
& \leq c_{2}\left\|Z_{\alpha}\right\| \lambda^{-\frac{1}{4}}\left\|V_{0}\right\|_{2}\left|A Z_{\alpha}\right| \\
& \leq \frac{\nu}{8}\left|A Z_{\alpha}\right|^{2}+\frac{c_{2}^{2}}{\nu \sqrt{\lambda_{1}}}\left\|V_{0}\right\|_{2}^{2}\left\|Z_{\alpha}\right\|^{2}
\end{aligned}
$$

In each of (iii), (iv), and (v) below, it is Lemma 8.9 that is very crucial to obtain third inequalities.

(iii)

$$
\begin{aligned}
\left|\int\left[\left(\left\{\mathcal{R}_{\alpha}-I\right\} V_{\alpha} \cdot \nabla\right) V_{0}\right] \cdot A Z_{\alpha} d x\right| & \leq\left\|\left\{\mathcal{R}_{\alpha}-I\right\} V_{\alpha}\right\|_{L^{3}}\left\|\nabla V_{0}\right\|_{L^{6}}\left|A Z_{\alpha}\right| \\
& \leq c_{3}\left\|\left\{\mathcal{R}_{\alpha}-I\right\} V_{\alpha}\right\|_{\frac{1}{2}}\left\|V_{0}\right\|_{2}\left|A Z_{\alpha}\right| \\
& \leq c_{3} \epsilon\left\|V_{\alpha}\right\|\left\|V_{0}\right\|_{2}\left|A Z_{\alpha}\right| \\
& \leq \frac{\nu}{8}\left|A Z_{\alpha}\right|^{2}+\frac{2 \epsilon^{2} c_{3}^{2}}{\nu}\left\|V_{\alpha}\right\|^{2}\left\|V_{0}\right\|_{2}^{2} .
\end{aligned}
$$


(iv)

$$
\begin{aligned}
\left|\int\left[V_{\alpha j} \nabla\left(\left\{R_{\alpha}-I\right\} V_{\alpha}\right)_{j}\right] \cdot A Z_{\alpha} d x\right| & =\left|\int\left[\left(A Z_{\alpha} \cdot \nabla\right) V_{\alpha}\right] \cdot\left\{R_{\alpha}-I\right\} V_{\alpha} d x\right| \\
& \leq\left\|\left\{R_{\alpha}-I\right\} V_{\alpha}\right\|_{L^{3}}\left\|\nabla V_{\alpha}\right\|_{L^{6}}\left|A Z_{\alpha}\right| \\
& \leq c_{4}\left\|\left\{R_{\alpha}-I\right\} V_{\alpha}\right\|_{\frac{1}{2}}\left\|V_{\alpha}\right\|_{2}\left|A Z_{\alpha}\right| \\
& \leq c_{4} \epsilon\left\|V_{\alpha}\right\|\left\|V_{\alpha}\right\|_{2}\left|A Z_{\alpha}\right| \\
& \leq \frac{\nu}{8}\left|A Z_{\alpha}\right|^{2}+\frac{2 \epsilon^{2} c_{4}^{2}}{\nu}\left\|V_{\alpha}\right\|^{2}\left\|V_{\alpha}\right\|_{2}^{2}
\end{aligned}
$$

(v)

$$
\begin{aligned}
\left|\int\left[f P_{L} J P_{L}\left(\mathcal{R}_{\alpha} V_{\alpha}-V_{0}\right)\right] \cdot A Z_{\alpha} d x\right| \leq & \left|\int\left[f P_{L} J P_{L}\left(\mathcal{R}_{\alpha}-I\right) V_{\alpha}\right] \cdot A Z_{\alpha} d x\right| \\
& +\left|\int f P_{L} J P_{L} Z_{\alpha} \cdot A Z_{\alpha} d x\right| \\
= & \left|\int\left[f P_{L} J P_{L}\left(\mathcal{R}_{\alpha}-I\right) V_{\alpha}\right] \cdot A Z_{\alpha} d x\right| \\
\leq & f\left|\left(\mathcal{R}_{\alpha}-I\right) V_{\alpha}\right|\left|A Z_{\alpha}\right| \\
\leq & \epsilon f\left\|V_{\alpha}\right\|\left|A Z_{\alpha}\right| \\
\leq & \frac{\nu}{8}\left|A Z_{\alpha}\right|^{2}+\frac{2 \epsilon^{2} f^{2}}{\nu}\left\|V_{\alpha}\right\|^{2} .
\end{aligned}
$$

Collecting all the estimates, we obtain

$$
\begin{aligned}
\frac{\partial}{\partial t}\left\|Z_{\alpha}\right\|^{2}+\frac{3 \nu}{4}\left|A Z_{\alpha}\right|^{2} \leq & \frac{c}{\nu^{3}}\left[\left\|V_{\alpha}\right\|^{4}+\frac{\nu^{2}}{\sqrt{\lambda_{1}}}\left\|V_{0}\right\|_{2}^{2}\right]\left\|Z_{\alpha}\right\|^{2} \\
& +\frac{\epsilon^{2} c}{\nu}\left\|V_{\alpha}\right\|^{2}\left[\left\|V_{0}\right\|_{2}^{2}+\left\|V_{\alpha}\right\|_{2}^{2}\right]+\frac{4 \epsilon^{2} f^{2}}{\nu}\left\|V_{\alpha}\right\|^{2}
\end{aligned}
$$

where $c^{2}=\max \left\{108 c_{1}^{4}, 4 c_{2}^{2}, 4 c_{3}^{2}, 4 c_{4}^{2}\right\}$ and $\epsilon$ arbitrary small number in Lemma 8.9. Write $y=\left\|Z_{\alpha}\right\|^{2}$. Then, from Eq. (C.1), we obtain,

$$
\frac{d y}{d t} \leq g y+h, \quad \forall t \in[0, T],
$$

where

$$
\begin{aligned}
& y=\left\|Z_{\alpha}\right\|^{2}=\left\|V_{\alpha}-V_{0}\right\| \\
& g=\frac{c}{\nu^{3}}\left[\left\|V_{\alpha}\right\|^{4}+\frac{\nu^{2}}{\sqrt{\lambda_{1}}}\left\|V_{0}\right\|_{2}^{2}\right] \equiv K_{4} \\
& h=\frac{\epsilon^{2} c}{\nu}\left\|V_{\alpha}\right\|^{2}\left[\left\|V_{0}\right\|_{2}^{2}+\left\|V_{\alpha}\right\|_{2}^{2}\right]+\frac{4 \epsilon^{2} f^{2}}{\nu}\left\|V_{\alpha}\right\|^{2} \equiv \epsilon^{2}\left(K_{5}+K_{3}\right) .
\end{aligned}
$$

Note that $K_{3}, K_{4}$ and $K_{5}$ are absolute constants on $[0, T]$. Let $T_{0}$ and $T_{\alpha 0}$ be entry times of the solutions of 3D rotating Navier-Stokes equations and 3D rotating NS$\alpha$ equations into absorbing balls, respectively. The rest of proof follows the line of Lemma 8.12. 


\section{REFERENCES}

[1] A. Babin, A. Mahalov and B. Nicolaenko, Long-time averaged Euler and Navier-Stokes equations for rotating fluids, in Structure and Dynamics of Nonlinear Waves in Fluids, Edited by K. Kirchgässner and A. Mielke, World Scientific, 145-157, 1995.

[2] A. Babin, A. Mahalov and B. Nicolaenko, Global splitting, integrability and regularity of $3 D$ Euler and Navier-Stokes equations for uniformly rotating fluids, European J. of Mechanics B/Fluids, 15, 291-300, 1996.

[3] A. Babin, A. Mahalov and B. Nicolaenko, Resonances and regularity for Boussinesq equations, Russian J. Math. Physics, 4, 417-428, 1996.

[4] A. Babin, A. Mahalov and B. Nicolaenko, Global splitting and regularity of rotating shallow-water equations, European J. of Mechanics B/Fluids, 16, 725-754, 1997.

[5] A. Babin, A. Mahalov and B. Nicolaenko, Regularity and integrability of rotating shallow-water equations, C. R. Acad. Sci. Paris: Series1, 324, 593-598, 1997.

[6] A. Babin, A. Mahalov and B. Nicolaenko, Regularity and integrability of 3D Euler and NavierStokes equations for rotating fluids, J. Asympt. Anal., 15, 103-150, 1997.

[7] A. Babin, A. Mahalov and B. Nicolaenko, Global regularity of 3D rotating Navier-Stokes equations for resonant domains, Indiana Univ. Math. J., 48, 1133-1176, 1999.

[8] A. Babin, A. Mahalov and B. Nicolaenko, On the regularity of three-dimensional rotating EulerBoussinesq equations, Math. Mod. and Appl. Sci., 9, 1089-1121, 1999.

[9] A. Babin, A. Mahalov and B. Nicolaenko, Fast singular oscillating limits and global regularity for the $3 D$ primitive equations of geophysics, Mathematical Modelling and Numerical Analysis, 34, 201-202, 2000.

[10] A. Babin, A. Mahalov and B. Nicolaenko, 3D Navier-Stokes equations with initial data characterized by uniformly large vorticity, Indiana Univ. Math. J., 50, 2001.

[11] A. Babin, A. Mahalov and B. Nicolaenko, Fast singular oscillating limits of stably-stratified $3 D$ euler and navier-stokes equations and ageostrophic wave fronts, Large-Scale AtmosphereOcean Dynamics 2, Newton Institute, Cambridge University Press, 2002.

[12] A. Babin, A. Mahalov and B. Nicolaenko, Global regularity of the 3D Navier-Stokes equations with uniformly large initial vorticity, Russian Math. Surveys (translation of Uspekhi Mat. Nauk), 58, 232-264, 2003.

[13] A. Babin, A. Mahalov, B. Nicolaenko and Y. Zhou, On the asymptotic regimes and the strongly stratified limit of rotating Boussinesq equations, Theor. and Comp. Fluid Dyn., 9, 223-251, 1997.

[14] S. Y. Chen, C. Foias, D. D. Holm, L. G. Margolin and R. Zhang, Direct numerical simulations of the Navier-Stokes alpha model, Physica D, 133, 66-83, 1999.

[15] S. Y. Chen, C. Foias, D. D. Holm, E. J. Olson, E. S. Titi and S. Wynne, Camassa-Holm equations as a closure model for turbulent channel and pipe flow, Phys. Rev. Lett., 81, 5338-5341, 1998.

[16] S. Y. Chen, C. Foias, D. D. Holm, E. J. Olson, E. S. Titi and S. Wynne, A connection between the Camassa-Holm equations and turbulent flows, Physics of Fluids, 11, 2343-2353, 1999.

[17] S. Y. Chen, C. Foias, D. D. Holm, E. J. Olson, E. S. Titi and S. Wynne, The Camassa-Holm equations and turbulence in pipes and channels, Physica D, 133, 49-65, 1999.

[18] P. Constantin and C. Foias, Navier-Stokes Equations, The University of Chicago Press, 1988.

[19] A. Eden, C. Foias, B. Nicolaenko and R. Temam, Exponential Attractors for Evolution Equations, John Wiley and Masson, 1994.

[20] B. Fabijonas and D. D. Holm, Mean effects of turbulence on elliptical instability, Phys. Rev. Lett., 90, 124501-124504, 2003.

[21] C. Foias, D. D. Holm and E. S. Titi, The Navier-Stokes-alpha model of fluid turbulence, Physica D, 152, 505-519, 2001.

[22] C. Foias, D. D. Holm and E. S. Titi, The three dimensional viscous Camassa-Holm equations, and their relation to the Navier-Stokes equations and turbulence theory, J. Dynamics and Differential Equations, 14, 1-35, 2002.

[23] J. K. Hale, Asymptotic Behavior of Dissipative Systems, AMS, 1988.

[24] D. D. Holm and B. Nadiga, Modeling mesoscale turbulence in the barotropic double gyre circulation, J. Physical Oceanography, 33, 2355-2365, 2003.

[25] D. D. Holm, J. E. Marsden and T. S. Ratiu, Euler-Poincaré models of ideal fluids with nonlinear dispersion, Phys. Rev. Lett., 80, 4173-4176, 1998.

[26] D. D. Holm, J. E. Marsden and T. S. Ratiu, Euler-Poincaré equations and semidirect products with applications to continuum theories, Adv. Math., 137, 1-81, 1998.

[27] D. D. Holm, J. E. Marsden and T. S. Ratiu, The Euler-Poincaré equations in geophysical fluid dynamics, In Large-Scale Atmosphere-Ocean Dynamics 2: Geometric Methods and Models, Edited by J. Norbury and I. Roulstone, Cambridge University Press, UK, 251-300, 2002. 
[28] B. Kim, Alpha Models for Rotating Navier-Stokes Equations in Geophysics with Nonlinear Dispersive Regularization, Ph.D Dissertation, Arizona State University, 2003.

[29] O. A. Ladyzhenskaya, The Mathematical Theory of Viscous Incompressible Flow, Gordon and Breach, 1963.

[30] J. Leray, Sur le mouvement d'un liquide visquex emplissent l'espace, Acta Math. J., 63, 193-248, 1934.

[31] K. Mohseni, B. Kosovic, S. Shkoller and J. Marsden, Numerical simulations of the Lagrangian averaged Navier-Stokes (LANS) equations for homogeneous isotropic turbulence, Phys. of Fluids, 15, 524-544, 2003.

[32] H. Poincaré, Sur la précession des corps déformables, Bull. Astronomique, 27, 321-356, 1910

[33] J. C. Robinson, Infinite-Dimensional Dynamical Systems, Cambridge University Press, 2001.

[34] S. L. Sobolev, Ob odnoi novoi zadache mathematicheskoi fiziki, Izvestiia Akademii Nauk SSSR, Ser. Mathematicheskaia, 18, 3-50, 1954.

[35] R. Temam, Navier-Stokes Equations: Theory and Numerical Analysis, North-Holand, 1984.

[36] R. Temam, Navier-Stokes Equations and Nonlinear Functional Analysis, SIAM, 1995.

[37] R. Temam, Infinite-Dimensional Dynamical Systems in Mechanics and Physics (2e), Springer, 1997.

[38] M. Trahe, Attractors for 3D Fast Rotating Navier-Stokes equations, Ph.D Dissertation, Arizona State University, 2002. 